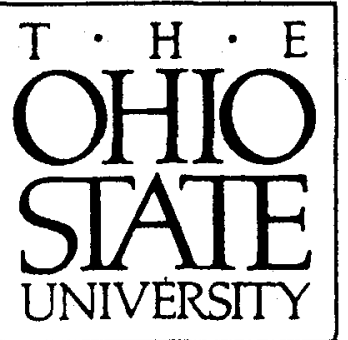

\title{
Analysis of Modified SMI Method for Adaptive Array Weight Control
}

\author{
R.L. Dilsavor and R.L. Moses
}

\section{The Ohio State University ElectroScience Laboratory \\ Department of Eloctrical Engineering \\ Columbus, Ohio $\mathbf{4 3 2 1 2}$}

Technical Report 716111-6

Contract No. NAG 3-536

February 1989

National Aeronautics and Space Administration

Lewis Research Center 21000 Brookpark Road

Cleveland, Ohio 44135

(AASA-CF-1E4C(4) ADAIYSIS CF ECEIFIED SUI BETHCL FCE ALARIIIE AEEAY LEIGET CCATEOI (Chio state CEIV.) \&E 


\section{NOTICES}

When Government drawings, specifications, or other data are used for any purpose other than in connection with a definitely related Government procurement operation, the United States Government thereby incurs no responsibility nor any obligation whatsoever, and the fact that the Government may have formulated, furnished, or in any way supplied the said drawings, specifications, or other data, is not to be regarded by implication or otherwise as in any manner licensing the holder or any other person or corporation, or conveying any rights or permission to manufacture, use, or sell any patented invention that may in any way be related thereto. 


\begin{tabular}{|c|c|c|}
\hline $\begin{array}{l}\text { REPORT DOCUMENTATION } \\
\text { PAGE }\end{array}$ & 1. REPORT NO. & 3. Recipient's Accession No. \\
\hline \multirow{2}{*}{\multicolumn{2}{|c|}{$\begin{array}{l}\text { 4. Title and Subtitle } \\
\text { Analysis of Modified SMI Method for Adaptive Array Weight Control }\end{array}$}} & $\begin{array}{l}\text { B. Report Date } \\
\text { February } 1989\end{array}$ \\
\hline & & 6. \\
\hline \multicolumn{2}{|c|}{$\begin{array}{l}\text { T. Author(s) } \\
\text { R.L. Dilsavor and R.L. Moses }\end{array}$} & $\begin{array}{l}\text { 8. Performing Org. Rept. No. } \\
716111-6\end{array}$ \\
\hline \multicolumn{2}{|c|}{$\begin{array}{l}\text { 9. Performing Organisation Name and Address } \\
\text { The Ohio State University }\end{array}$} & 10. Project/Task/Work Unit No. \\
\hline $\begin{array}{l}\text { ElectroScience Laboratory } \\
1320 \text { Kinnear Road } \\
\text { Columbus, OH } 43212 \\
\end{array}$ & & $\begin{array}{l}\text { 11. Contract(C) or Grant(G) No. } \\
\text { (C) NAG s-636 } \\
\text { (G) }\end{array}$ \\
\hline \multicolumn{2}{|l|}{ NASA-Lewis Research Center } & $\begin{array}{l}\text { 13. Report Type/Period Covered } \\
\text { Technical }\end{array}$ \\
\hline \multicolumn{2}{|l|}{21000 Brookpark Road } & 14. \\
\hline
\end{tabular}

15. Supplementary Notes

16. Abstract (Limit: 200 words)

An adaptive array is applied to the problem of receiving a desired signal in the presence of weak interference signals which need to be suppressed. A modification, suggested by Gupta, of the sample matrix inversion (SMI) algorithm controls the array weights. In the modified SMI algorithm, interference suppression is increased by subtracting a fraction $F$ of the noise power from the diagonal elements of the estimated covariance matrix. Given the true covariance matrix and the desired signal direction, the modified algorithm is shown to maximize a well-defined, intuitive output power ratio criterion. Expressions are derived for the expected value and variance of the array weights and output powers as a function of the fraction $F$ and the number of snapshots used in the covariance matrix estimate. These expressions are compared with computer simulation and good agreement is found. A tradeoff is found to exist between the desired level of interference suppression and the number of snapshots required in order to achieve that level with some certainty. The removal of foise eigenvectors from the covariance matrix inverse is also discussed with respect to this application. Finally, the type and severity of errors which occur in the covariance matrix estimate are characterized through simulation.

17. Document Analysis a. Descriptors

b. Identiflers/Open-Ended Terms

c. COSATI Field/Group

18. A vailability Statement

18. Security Class (This Report)
Unclassified

20. Security Class (This Page)

Unclassified 


\section{Contents}

LIST OF FIGURES

iv

CHAPTER

PAGE

1 Introduction 1

2 Modification of SMI Algorithm and Pertinent Theory 6

2.1 Theoretical Model and Modification of SMI . . . . . . . 7

2.2 Theoretical Performance of the Modified SMI Algorithm . . 13

2.3 Covariance Matrix Eigenstructure . . . . . . . . . . . 14

2.4 Instructive Example . . . . . . . . . . . . . . 19

2.5 Summary ................... 20

3 Modified SMI Array Performance with Estimated Covariance 21

3.1 Sample Covariance Matrix . . . . . . . . . . . . 22

3.2 Statistical Analysis Results . . . . . . . . . . . . 22

3.3 Computer Simulation and Observations . . . . . . . . 28

3.3.1 Signal Snapshot Model . . . . . . . . . . . . . 28

3.3.2 Interpreting the Plots . . . . . . . . . . . . 30

3.3.3 Estimating the Noise Power . . . . . . . . . . . 32

3.3.4 Varying the Fraction F . . . . . . . . . . . 34

3.3.5 Omitting Noise Eigenvectors from the Weight Expression . . . . . . . . . . . . 45

3.3.6 Characterization of Sample Covariance Errors . . . . 54

3.4 Summary ................... 72

4 Conclusions $\quad 76$

iii 


\section{List of Figures}

1.1 Ground station receiving desired satellite signal in presence of weak interference signals. . . . . . . . . . . . . .

2.1 An $\mathrm{N}$-element array receiving a desired signal and $\mathrm{M}$ interference signals. . . . . . . . . . . . .

2.2 Adaptive antenna array with 4 auxiliary elements receiving a desired signal from broadside and a weak interference signal from $30^{\circ}$ off broadside. . . . . . . . . . . . . . .

2.3 Output INR and SINR of the 4-auxiliary element adaptive array versus fraction $F$. SNR(main) $=14.6 \mathrm{~dB}, \mathrm{SNR}$ (aux) $=-10 \mathrm{~dB}, \operatorname{INR}($ main $)=-5 \mathrm{~dB}, \operatorname{INR}($ aux $)=-3 \mathrm{~dB}, \theta_{D}=0^{\circ}$, $\theta_{I 1}=30^{\circ} \ldots \ldots \ldots \ldots \ldots \ldots \ldots$

3.1 Six trials showing the minimum eigenvalue of $\hat{\Phi}_{K}$ approaching the true noise variance $\sigma^{2}=1$ as the number of snapshots $\mathrm{K}$ in the covariance estimate increases. . . . . . . . .

3.2 Plot of output INR and SINR versus number of snapshots $K$ for $F=0$ shows 4 trials and straight line representing the infinite data (true covariance) case. . . . . . . . . . .

3.3 Plot of output INR and SINR versus number of snapshots $K$ for $F=0.8$ shows 4 trials and straight line representing the infinite data (true covariance) case. . . . . . . . .

3.4 Plot of output INR and SINR versus number of snapshots $K$ for $F=0.9$ shows 4 trials and straight line representing the infinite data (true covariance) case. . . . . . . . . .

3.5 Plot of output desired signal power $P_{D}$ versus number of snapshots $K$ for $F=0.0$ shows 4 trials, true covariance curve, bias curve, and $95 \%$ confidence interval. . . . . . 
3.6 Plot of output desired signal power $P_{D}$ versus number of snapshots $K$ for $F=0.8$ shows 4 trials, true covariance curve, bias curve, and $95 \%$ confidence interval. . . . . . .

3.7 Plot of output desired signal power $P_{D}$ versus number of snapshots $K$ for $F=0.9$ shows 4 trials, true covariance curve, bias curve, and $95 \%$ confidence interval. . . . . . .

3.8 Plot of output interference signal power $P_{I}$ versus number of snapshots $K$ for $F=0.0$ shows 4 trials, true covariance curve, bias curve, and $95 \%$ confidence interval. . . . . . .

3.9 Plot of output interference signal power $P_{I}$ versus number of snapshots $K$ for $F=0.8$ shows 4 trials, true covariance curve, bias curve, and $95 \%$ confidence interval. . . . . . .

3.10 Plot of output interference signal power $P_{I}$ versus number of snapshots $K$ for $F=0.9$ shows 4 trials, true covariance curve, bias curve, and $95 \%$ confidence interval. . . . . . .

3.11 Plot of output noise power $P_{\eta}$ versus number of snapshots $K$ for $F=0.0$ shows 4 trials, true covariance curve, bias curve, and $95 \%$ confidence interval. . . . . . . . . . .

3.12 Plot of output noise power $P_{\eta}$ versus number of snapshots $K$ for $F=0.8$ shows 4 trials, true covariance curve, bias curve, and $95 \%$ confidence interval. . . . . . . . . . . .

3.13 Plot of output noise power $P_{\eta}$ versus number of snapshots $K$ for $F=0.9$ shows 4 trials, true covariance curve, bias curve, and $95 \%$ confidence interval. . . . . . . . . . .

3.14 Real and imaginary parts of main element weight $W_{1}$ versus number of snapshots $K$ for $F=0.8$ shows 4 trials, true covariance curve, bias curve, and $95 \%$ confidence interval. All eigenvectors are used in the weight expression. . . . . .

3.15 Real and imaginary parts of first auxiliary element weight $W_{2}$ versus number of snapshots $K$ for $F=0.8$ shows 4 trials, true covariance curve, bias curve, and $95 \%$ confidence interval. All eigenvectors are used in the weight expression. . . . . .

3.16 Real and imaginary parts of main element weight $W_{1}$ versus number of snapshots $K$ for $F=0.8$ shows 4 trials and true covariance curve. Only principal eigenvectors are used in the weight expression. ............... 
3.17 Real and imaginary parts of first auxiliary element weight $W_{2}$ versus number of snapshots $K$ for $F=0.8$ shows 4 trials and true covariance curve. Only principal eigenvectors are used in the weight expression. . . . . . . . . . . . .

3.18 Plot of output desired signal power $P_{D}$ versus number of snapshots $K$ for $F=0.8$ shows 4 trials and the true covariance curve. Weights were found using only the signal

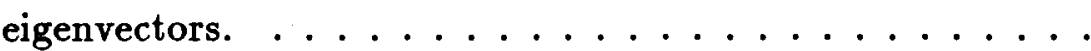

3.19 Plot of output interference signal power $P_{I}$ versus number of snapshots $K$ for $F=0.8$ shows 4 trials and the true covariance curve. Weights were found using only the signal eigenvectors. . . . . . . . . . . . . .

3.20 Plot of output noise power $P_{\eta}$ versus number of snapshots $K$ for $F=0.8$ shows 4 trials and the true covariance curve. Weights were found using only the signal eigenvectors. . . .

3.21 Plot of output INR and SINR versus number of snapshots $K$ for $F=0.8$ and $\hat{\Phi}_{K}=\Phi_{D}+\Phi_{I 1}+(1 / K) \sum_{k=1}^{K} X_{\eta k} X_{\eta k}^{H}$. Shows 4 simulations and the true covariance curve. . . . . .

3.22 Plot of output desired signal power $P_{D}$ versus number of snapshots $K$ for $F=0.8$ and $\hat{\Phi}_{K}=\Phi_{D}+\Phi_{I 1}+(1 / K) \sum_{k=1}^{K} X_{\eta k} X_{\eta k}^{H}$. Shows 4 simulations and the true covariance curve. . . . . 60

3.23 Plot of output interference signal power $P_{I}$ versus number of snapshots $K$ for $F=0.8$ and $\hat{\Phi}_{K}=\Phi_{D}+\Phi_{I 1}+(1 / K) \sum_{k=1}^{K} X_{\eta k} X_{\eta k}^{H}$. Shows 4 simulations and the true covariance curve. . . . .

3.24 Plot of output noise power $P_{\eta}$ versus number of snapshots $K$ for $F=0.8$ and $\hat{\Phi}_{K}=\Phi_{D}+\Phi_{I 1}+(1 / K) \sum_{k=1}^{K} X_{\eta k} X_{\eta k}^{H}$. Shows 4 simulations and the true covariance curve. . . . .

3.25 Plot of output INR and SINR versus number of snapshots $K$ for $F=0.8$ and $\hat{\Phi}_{K}=\Phi+$ DICT. Shows 4 simulations and the true covariance curve. . . . . . . . . .

3.26 Plot of output desired signal power versus number of snapshots $K$ for $F=0.8$ and $\hat{\Phi}_{K}=\Phi+$ DICT. Shows 4 simulations and the true covariance curve. . . . . . . . . .

3.27 Plot of output interference power versus number of snapshots $K$ for $F=0.8$ and $\hat{\Phi}_{K}=\Phi+$ DICT. Shows 4 simulations and the true covariance curve. 
3.28 Plot of output noise power versus number of snapshots $K$ for $F=0.8$ and $\hat{\Phi}_{K}=\Phi+$ DICT. Shows 4 simulations and the true covariance curve. . . . . . . . . . . .

3.29 Plot of output INR and SINR versus number of snapshots $K$ for $F=0.8$ and $\hat{\Phi}_{K}=\Phi+$ DNCT. Shows 4 simulations and the true covariance curve. . . . . . . . . .

3.30 Plot of output desired signal power versus number of snapshots $K$ for $F=0.8$ and $\hat{\Phi}_{K}=\Phi+$ DNCT. Shows 4 simulations and the true covariance curve. . . . . . . . .

3.31 Plot of output interference power versus number of snapshots $K$ for $F=0.8$ and $\hat{\Phi}_{K}=\Phi+$ DNCT. Shows 4 simulations and the true covariance curve. . . . . . . . . .

3.32 Plot of output noise power versus number of snapshots $K$ for $F=0.8$ and $\hat{\Phi}_{K}=\Phi+$ DNCT. Shows 4 simulations and the true covariance curve. . . . . . . . . . . .

3.33 Plot of output INR and SINR versus number of snapshots $K$ for $F=0.8$ and $\hat{\Phi}_{K}=\Phi+$ INCT. Shows 4 simulations and the true covariance curve. . . . . . . . . .

3.34 Plot of output INR and SINR versus number of snapshots $K$ for $F=0.8$ and $\hat{\Phi}_{K}$ simulated normally (all crossterms) but with its first column and row replaced with those of the true covariance $\Phi$. Shows 4 simulations and the true covariance

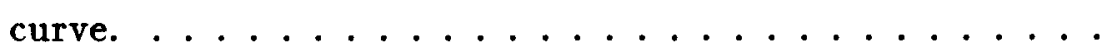




\section{Chapter 1}

\section{Introduction}

The ability of adaptive antenna arrays to form controllable, time-variable antenna patterns allows them to track moving desired-signal sources and to suppress signals from moving sources of accidental or intentional interference. This flexibility in the antenna pattern is achieved by summing the weighted outputs of a number of antenna elements. The instantaneous values of the time-varying weights determine the instantaneous array antenna pattern. The weights are varied according to an assignment rule or feedback control law. Different types of adaptive arrays are defined by the type of assignment rule or control law used to set the weights. The type of adaptive array which should be used in a particular application depends on that application.

In this work, the adaptive array is applied to the reception of a desired signal in the presence of weak interference signals that need to be suppressed. In particular, the reception at a ground station of a desired satellite signal in the presence of noise and undesired interference signals from neighboring satellites is considered. Figure 1.1 illustrates the situa- 
tion. An equivalent problem is that of a satellite receiving a desired signal from one of a number of neighboring ground stations. Adaptive arrays are applicable since, without adaptation at the receive site, interference signals may enter through the receive antenna sidelobes. Furthermore, the satellite orbits are not perfectly geostationary and more satellites and ground stations will enter the signal environment in the future. As seen in Figure 1.1 , the interfering signals enter the receive system through the sidelobes of the main antenna causing the input signal-to-interference ratio (SIR) to be in the range 10 to $30 \mathrm{~dB}$. Typically, the signal-to-noise ratio (SNR) is $15 \mathrm{~dB}$. Thus, the interfering signal is weak compared to the desired signal and may be several $\mathrm{dB}$ below the noise level.

Though the interference is weak, its presence is arguably more irritating than that of noise because of the similarity in the frequency content of the desired and interfering signals. For example, in the case of television signals, an interfering station may cause wavy lines or ghost images in the television picture in comparison with the less irritating "snow" associated with random noise. Thus, there is a special need to suppress weak interfering signals.

It is appropriate to choose the steered-beam type array for this application because the desired signal direction is known and assumed fixed. An array which uses the conventional sample matrix inversion (SMI) algorithm as its weight assignment rule is one such steered-beam type array. The SMI algorithm was derived so as to maximize the ratio of desired to undesired signal powers at the array output under steady state conditions, and given the desired signal direction. The undesired signal consists of noise and in- 


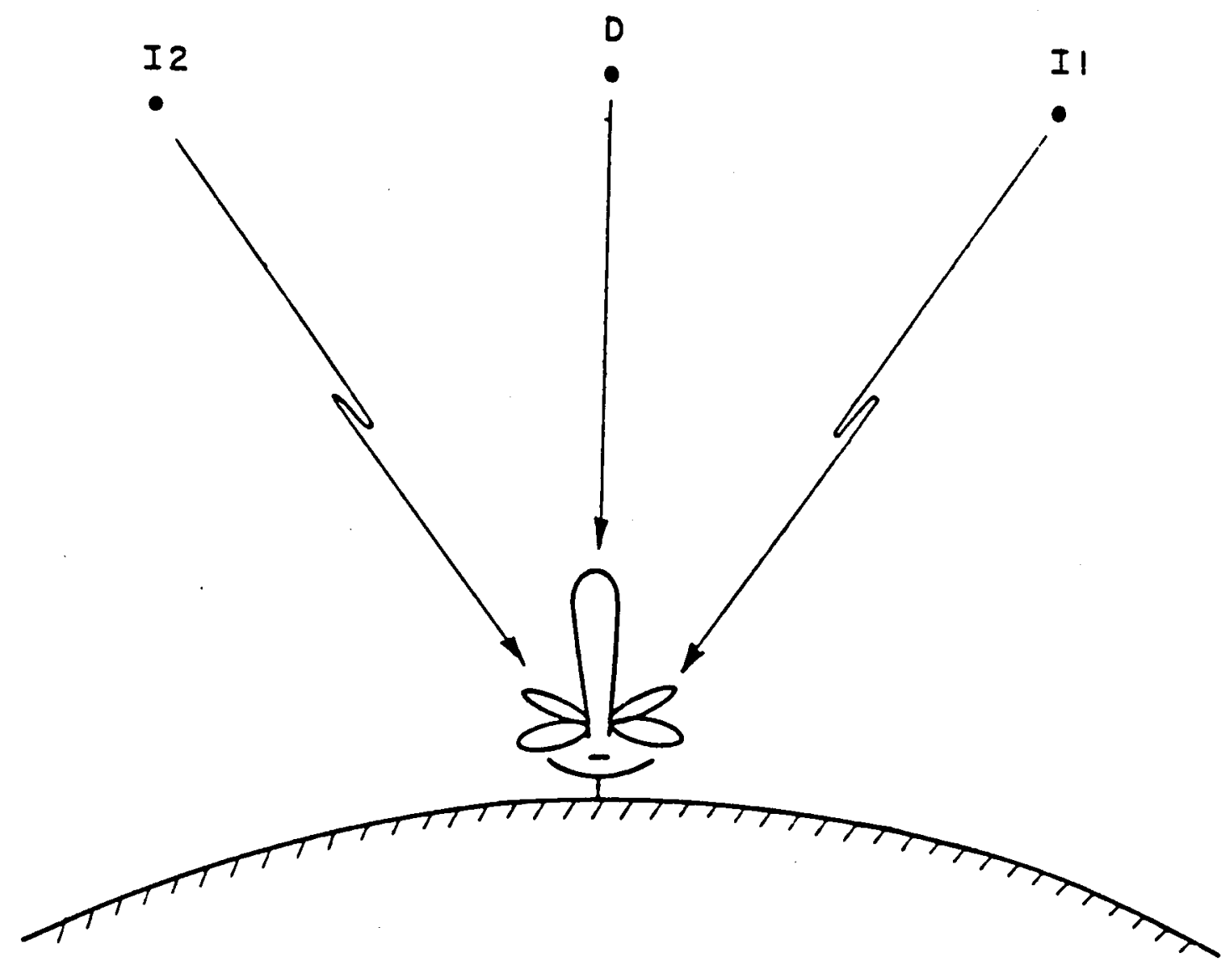

Figure 1.1: Ground station receiving desired satellite signal in presence of weak interference signals. 
terference components. In other words, conventional SMI adaptive arrays try to maximize the signal-to-interference-plus-noise ratio (SINR) as the signal environment changes. Indeed, the environment changes slowly in this application.

The problem with using conventional SMI is that maximum SINR is not desired since the presence of interference has been deemed more costly than that of noise. It is desired to unequally weight the contributions of the interference and noise components in the power ratio to be optimized in order to emphasize the importance given to suppressing the interference. The modified SMI algorithm discussed in this report maximizes a modified SINR (MSINR). The denominator of the MSINR is equal to the interference power plus only a fraction of the noise power.

This report extends the work of Gupta [1] who proposed the modified SMI algorithm and showed that the desired interference is theoretically attainable. In Chapter 2, the conventional SMI algorithm is modified in order to maximize the MSINR and the theoretical performance of a modified SMI array is presented. In addition, a geometric interpretation is obtained by formulating the algorithm in terms of the eigenstructure of the covariance matrix of received signals. A simple example to illustrate the effect of modifying the algorithm concludes Chapter 2. An actual SMI array system makes estimates of a covariance matrix based on a finite number of signal samples. This estimation degrades the performance of the real antenna system relative to the theoretical performance of Chapter 2. Chapter 3 describes a computer simulation of real array performance, presents results of a statistical analysis of this performance, and investigates, through sim- 
ulations, the nature of the weak interference suppression problem. The last chapter draws conclusions about the modified SMI algorithm and suggests a future course of action. 


\section{Chapter 2}

\section{Modification of SMI Algorithm and Pertinent Theory}

In this chapter, the standard SMI algorithm for adaptive array weight control is presented and modified in order to maximize the MSINR given the steering vector (i.e. desired signal direction, element gains in that direction, and array geometry). The modified SMI algorithm is shown to yield increased interference suppression when the true received-signal covariance matrix is assumed known. The chapter begins by introducing the theoretical array and signal model. The standard SMI algorithm is stated and modified in order to theoretically increase interference suppression. The eigen-decomposition of the covariance matrix which yields a geometric interpretation of the algorithm is studied in detail. A simple example concludes the chapter. Throughout the derivations, complex signal notation shall be used. Real signals are obtainable as the real part of the corresponding complex signal. 


\subsection{Theoretical Model and Modification of SMI}

Figure 2.1 represents an antenna array with $\mathrm{N}$ elements receiving a desired signal and $M$ interfering signals. Also present at each element is zero-mean complex Gaussian white noise with power $\sigma^{2}$. The modified SMI algorithm introduced later in this section relies on the fact that the smallest eigenvalue of the received-signal covariance matrix is an estimate of the noise power $\sigma^{2}$ at each element of the array if the dimension of the matrix is greater than the total number of received signals. Thus, it is assumed that $M \leq N-2$. The signal received at the $n^{\text {th }}$ antenna element is

$x_{n}(t)=a_{D n} \exp \left[j\left(\omega_{D} t+\phi_{D n}\right)\right]+\sum_{m=1}^{M} a_{I m n} \exp \left[j\left(\omega_{I m} t+\phi_{I m n}+\psi_{I m}\right)\right]+\eta_{n}(t)$

for $n=1,2, \ldots, N$. The amplitude, frequency, and phase of the desired signal at the $n^{\text {th }}$ element of the array are $a_{D_{n}}, \omega_{D}$, and $\phi_{D_{n}}$, respectively. $a_{I m n}, \omega_{I m}$, and $\phi_{I m n}$, are analogous parameters for the $m^{\text {th }}$ interference signal. The amplitudes depend on $n$ since the antenna elements may not have identical patterns. Zero phase reference has been chosen as the phase of the desired signal in the first element, thus $\phi_{D 1}=0^{\circ}$. Specifically, $\phi_{I m n}$ is the phase of the $m^{\text {th }}$ interference signal in the $n^{\text {th }}$ element with respect to its phase in the first element. Also $\psi_{I m}$ is the phase of the $m^{\text {th }}$ interference signal in the first element with respect to the phase reference and is assumed to be a uniform random variable on the interval $[0,2 \pi]$. The $\eta_{n}(t)$ represents the noise at the $n^{\text {th }}$ element and is a member of the ensemble of complex Gaussian white noise processes with power equal to $\sigma^{2}$. All ran- 
dom variables are assumed to be statistically independent. The narrowband approximation has been made in that each signal has been represented by a single frequency. The phase shifts $\phi_{J_{n}}$ result from the spatial separation of the antenna elements and are given by

$$
\phi_{J_{n}}=2 \pi(n-1) \frac{d}{\lambda} \sin \left(\theta_{J}\right) \quad J=D \text { or } I_{m}
$$

for a linear array with equally spaced elements; $d$ is the element separation, $\lambda$ is the signal wavelength, and $\theta_{J}$ is the signal arrival angle measured from broadside.

The signals received at each element can be combined in a single $(N \times 1)$ signal vector

$$
X(t)=\left[\begin{array}{c}
x_{1}(t) \\
x_{2}(t) \\
\vdots \\
x_{N}(t)
\end{array}\right]=X_{D}(t)+\sum_{m=1}^{M} X_{I m}(t)+X_{\eta}(t)
$$

where

$$
\begin{aligned}
& X_{D}(t)=A_{D} U_{D} \exp \left[j \omega_{D} t\right] \\
& X_{I m}(t)=A_{I m} U_{I m} \exp \left[j\left(\omega_{I m} t+\psi_{I m}\right)\right] \text {, } \\
& X_{\eta}(t)=\left[\begin{array}{llll}
\eta_{1}(t) & \eta_{2}(t) & \cdots & \eta_{N}(t)
\end{array}\right]^{T}, \\
& A_{J}=\operatorname{diag}\left[\begin{array}{llll}
a_{J 1} & a_{J 2} & \cdots & a_{J N}
\end{array}\right], \text { and } \\
& U_{J}=\left[\begin{array}{llll}
\exp \left(-j \phi_{J 1}\right) & \exp \left(-j \phi_{J 2}\right) & \cdots & \exp \left(-j \phi_{J N}\right)
\end{array}\right]^{T}
\end{aligned}
$$

where $J=D$ or $I_{m}$ and ${ }^{T}$ denotes transpose. The complex weights on the 


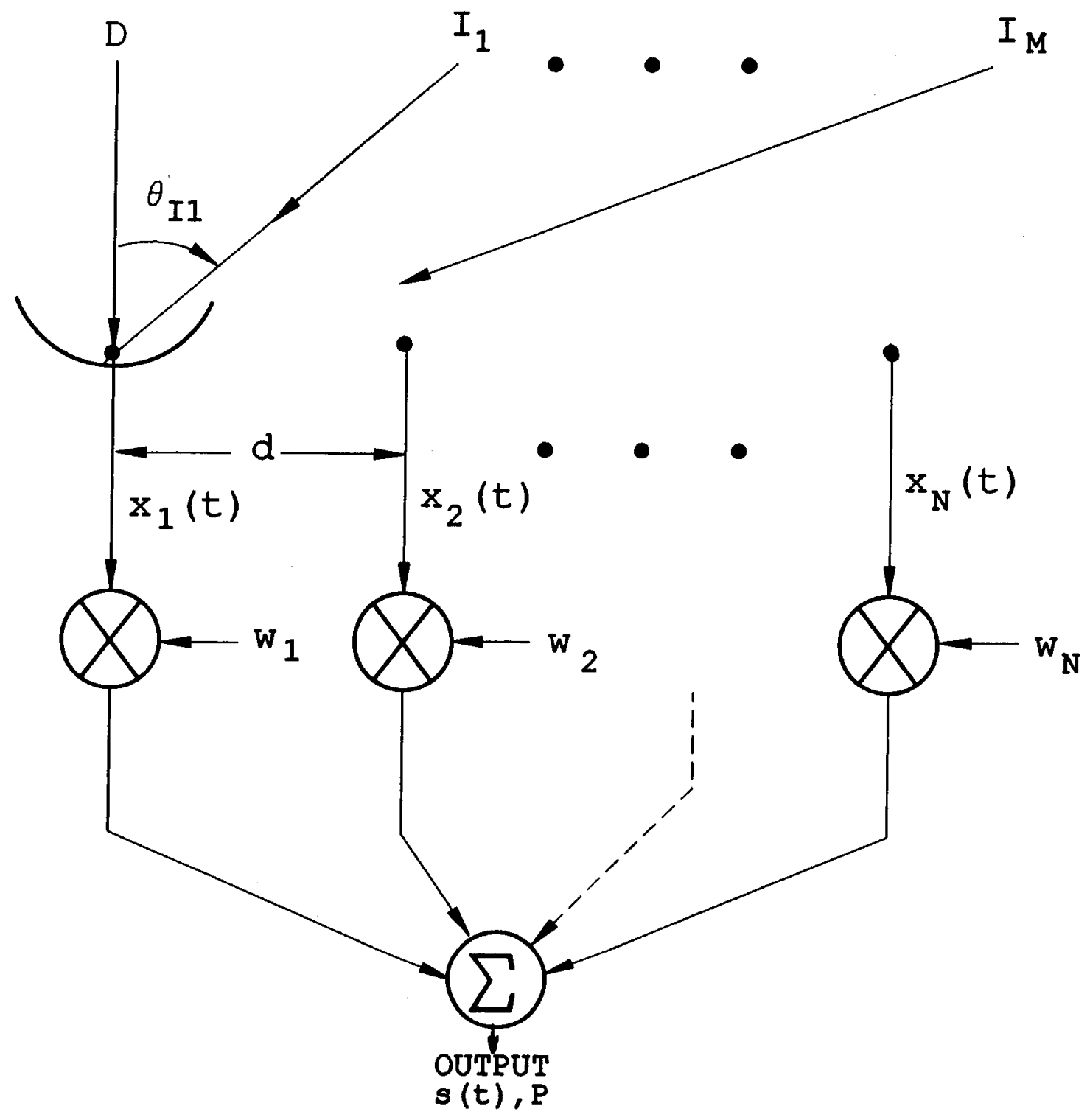

Figure 2.1: An $\mathrm{N}$-element array receiving a desired signal and $\mathrm{M}$ interference signals. 
antenna elements are also combined in an $(N \times 1)$ weight vector

$$
W(t)=\left[\begin{array}{c}
w_{1}(t) \\
w_{2}(t) \\
\vdots \\
w_{N}(t)
\end{array}\right]
$$

The received signals $X(t)$ are weighted and summed to form the array output signal shown in Figure 2.1

$$
s(t)=W^{H}(t) X(t)
$$

where ${ }^{H}$ denotes Hermitian transpose. By writing $X(t)$ using 2.3 , the output signal is expressed in terms of its components

$$
s(t)=s_{D}(t)+\sum_{m=1}^{M} s_{I m}(t)+s_{\eta}(t)
$$

where $s_{J}(t)=W^{H}(t) X_{J}(t)$ and $J=D, I m$, or $\eta$. Because of the random nature of the output signal the ensemble average or expectation operator is used to define the average power of the complex signal $s(t)$

$$
\begin{aligned}
P & =E\left[|s(t)|^{2}\right] \\
& =E\left[W^{H} X X^{H} W\right] \\
& =W^{H} E\left[X X^{H}\right] W \\
& =W^{H} \Phi W
\end{aligned}
$$

where $\Phi \equiv E\left[X X^{H}\right]$ is the $(N \times N)$ covariance matrix of the received signals. Notice that the time dependency of the weights $W$ and the received signals $X$ has been and will continue to be omitted for simplicity. Using 2.3 and 
the assumptions that the received signal components $X_{J}$ are uncorrelated and zero mean, the expectation used to define $\Phi$ evaluates to

$$
\begin{aligned}
\Phi & \equiv E\left[X X^{H}\right] \\
& =E\left[X_{D} X_{D}^{H}\right]+\left(\sum_{m=1}^{M} E\left[X_{I m} X_{I m}^{H}\right]\right)+E\left[X_{\eta} X_{\eta}^{H}\right] \\
& \equiv \Phi_{D}+\left(\sum_{m=1}^{M} \Phi_{I m}\right)+\Phi_{\eta} \\
& =S_{D} S_{D}^{H}+\left(\sum_{m=1}^{M} S_{I m} S_{I m}^{H}\right)+\sigma^{2} I
\end{aligned}
$$

where

$$
\begin{aligned}
\Phi_{D} & \equiv E\left[X_{D} X_{D}^{H}\right]=S_{D} S_{D}^{H}, \\
\Phi_{I m} & \equiv E\left[X_{I m} X_{I m}^{H}\right]=S_{I m} S_{I m}^{H}, \\
\Phi_{\eta} & \equiv E\left[X_{\eta} X_{\eta}^{H}\right]=\sigma^{2} I, \\
S_{D} & \equiv A_{D} U_{D}, \text { and } \\
S_{I m} & \equiv A_{I m} U_{I m}
\end{aligned}
$$

for $m=1,2, \ldots M$. The $I$ in 2.15 is an $(N \times N)$ identity matrix. We call $S_{D}$ the desired signal vector or steering vector and $S_{I m}$ the $m^{\text {th }}$ interference signal vector. Using 2.13 in 2.12 , the output power $P$ may be easily expressed as

$$
P=P_{D}+P_{I}+P_{\eta}
$$

where

$$
\begin{aligned}
P_{D} & =W^{H} \Phi_{D} W \\
P_{I} & =W^{H}\left(\sum_{m=1}^{M} \Phi_{I m}\right) W
\end{aligned}
$$




$$
\begin{aligned}
& =W^{H} \Phi_{I} W, \text { and } \\
P_{\eta} & =W^{H} \Phi_{\eta} W=\sigma^{2} W^{H} W .
\end{aligned}
$$

Equations 2.18 may be used to write the output SINR as

$$
\begin{aligned}
S I N R & =\frac{P_{D}}{P_{I}+P_{\eta}} \\
& =\frac{W^{H} \Phi_{D} W}{W^{H} \Phi_{I} W+\sigma^{2} W^{H} W} .
\end{aligned}
$$

In this application it is desired to maximize a modified SINR

$$
\begin{aligned}
M S I N R & =\frac{P_{D}}{P_{I}+(1-F) P_{\eta}} \\
& =\frac{W^{H} \Phi_{D} W}{W^{H} \Phi_{I} W+(1-F) \sigma^{2} W^{H} W} .
\end{aligned}
$$

where $0 \leq F \leq 1$. Note that the MSINR reduces to the SINR when $F=0$.

Reed et.al [2] showed that, given the steering vector $S_{D}$ defined in 2.16, maximum SINR is achieved by choosing the optimal weights as

$$
W_{S}=\mu \Phi^{-1} S_{D}=\mu\left[\Phi_{D}+\Phi_{I}+\sigma^{2} I\right]^{-1} S_{D}
$$

where subscript ${ }_{S}$ denotes "standard choice", and where $\mu$ is an arbitrary constant. Notice that the only difference between the SINR and MSINR of Equations 2.19 and 2.20 is that the constant (with respect to $W$ ) $\sigma^{2}$ has been replaced by the constant $(1-F) \sigma^{2}$. The weights that optimize the MSINR are easily obtained by adjusting the $\sigma^{2}$ of 2.21 accordingly;

$$
\begin{aligned}
W & =\mu\left[\Phi_{D}+\Phi_{I}+(1-F) \sigma^{2} I\right]^{-1} S_{D} \\
& =\mu\left[\Phi-F \sigma^{2} I\right]^{-1} S_{D} \\
& =\mu[\Gamma]^{-1} S_{D}
\end{aligned}
$$


where $\Gamma \equiv \Phi-F \sigma^{2} I$.

A critical result arrived at in Section 2.3 is that the noise power $\sigma^{2}$ is available as the minimum eigenvalue of $\Phi$ when the signal scenario consists of pure sinusoids in white noise incident upon an array with one or more unused degrees of freedom [3]. This fact makes possible the implementation of the modified weights of 2.22 on a real antenna system since the covariance matrix, and thus its minimum eigenvalue, can be estimated.

A number of observations can be made. Notice that the standard SMI algorithm is just a special case of the modified algorithm where $F=0$. As $F$ is allowed to approach 1 , the $\Gamma$ matrix approaches singularity, since $\operatorname{rank}\left(\Phi_{D}+\sum \Phi_{I m}\right) \leq M+1 \leq N-1$ where the first inequality follows from 2.15 and the second follows from assumption. Choosing $F>0$ will decrease the INR at the expense of necessarily decreasing the SINR since the choice of $F=0$ maximized SINR. It is expected that the gain in interference suppression will cause a loss in desired signal power and a gain in output noise power. The next section studies these gains and losses.

\subsection{Theoretical Performance of the Modi- fied SMI Algorithm}

Assuming that the signals received at the array elements are given exactly by 2.1 , the ensemble average that defines $\Phi$ in 2.16 can be taken and the steering vector may be found exactly. The minimum eigenvalue of $\Phi$ is $\sigma^{2}$ as shown in the next section. Using these parameters in 2.22 along with a chosen value of $F$ yields the modified weights. Once the weights 
are determined, any performance measure including the INR and the SINR can be found.

The modified SMI algorithm is applied to the practical antenna array shown in Figure 2.2 consisting of a high-gain main element and four auxiliary elements with half-wavelength spacing. A desired signal is incident from broadside while an interference signal arrives 30 degrees from broadside. The SNR of the desired signal is $14.6 \mathrm{~dB}$ in the main antenna while it is $-10 \mathrm{~dB}$ in the auxiliaries. The INR is $-5 \mathrm{~dB}$ in the main antenna and is $-3 \mathrm{~dB}$ in the auxiliaries. This is a good example of weak interference since the interference power is about $20 \mathrm{~dB}$ beneath the desired signal and is even a few $\mathrm{dB}$ below the noise. This scenario which is equivalent to that considered by Gupta [1] will also be used in the simulations of the next chapter.

A plot of INR and SINR versus the fraction $F$ is given in Figure 2.3. These plots, which support the work of Gupta [1], show that a significant decrease in INR can be achieved at the slight expense of a small decrease in SINR for a properly chosen value of fraction $F$ assuming perfect covariance information is available. For example, choosing $F=0.8$ yields $11.5 \mathrm{~dB}$ suppression of interference beneath the standard SMI level (the value when $F=0$ ) at the small cost of a $0.3 \mathrm{~dB}$ reduction in the SINR.

\subsection{Covariance Matrix Eigenstructure}

The eigen-decomposition of $\Phi$ is useful in obtaining a relationship between $\Gamma^{-1}$ and $\Phi^{-1}$ of 2.21 and 2.23. Let the positive real eigenvalues $\lambda_{i}$ of the 


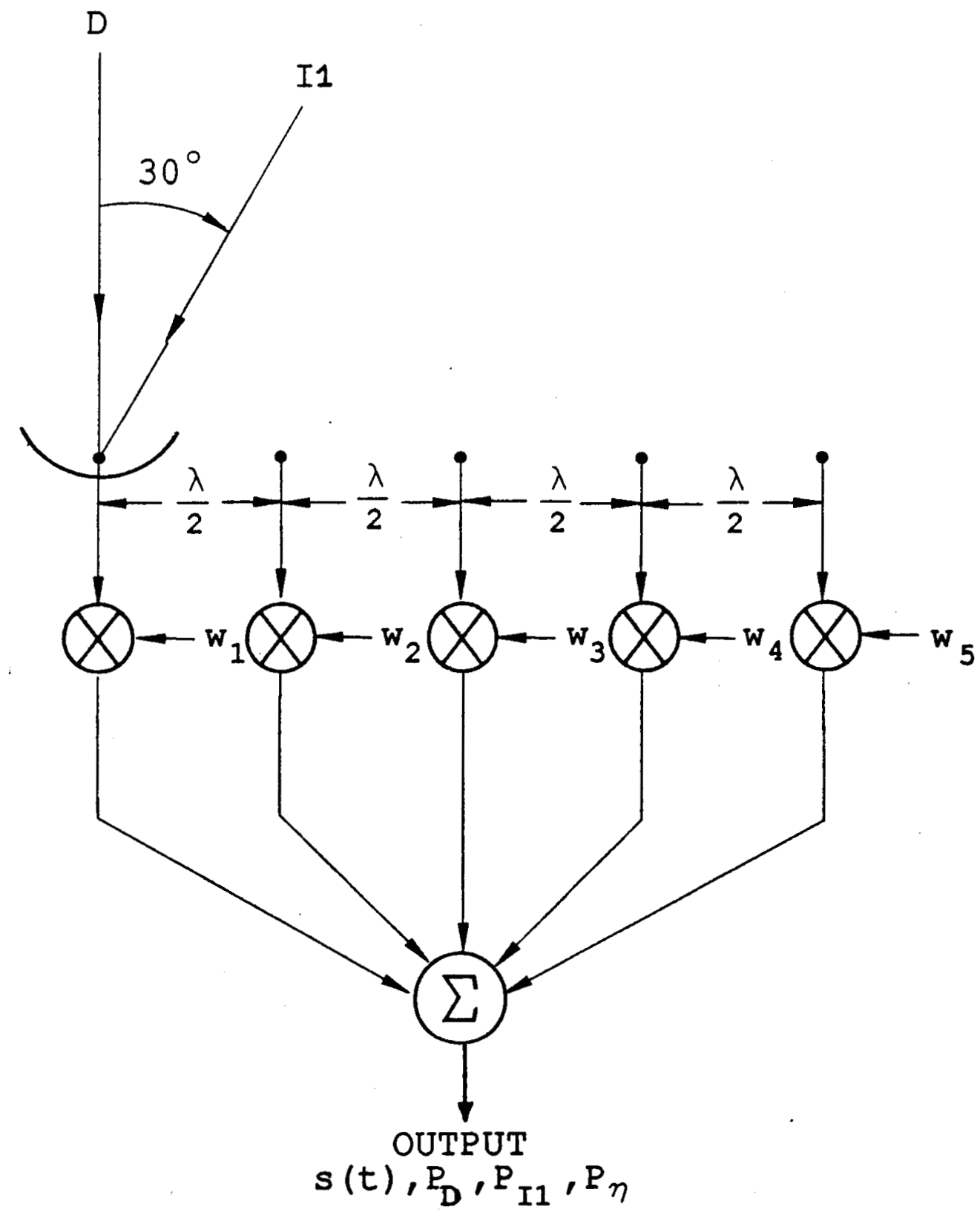

Figure 2.2: Adaptive antenna array with 4 auxiliary elements receiving a desired signal from broadside and a weak interference signal from $30^{\circ}$ off broadside. 
OUTPUT INR \& SINR VS FRAC

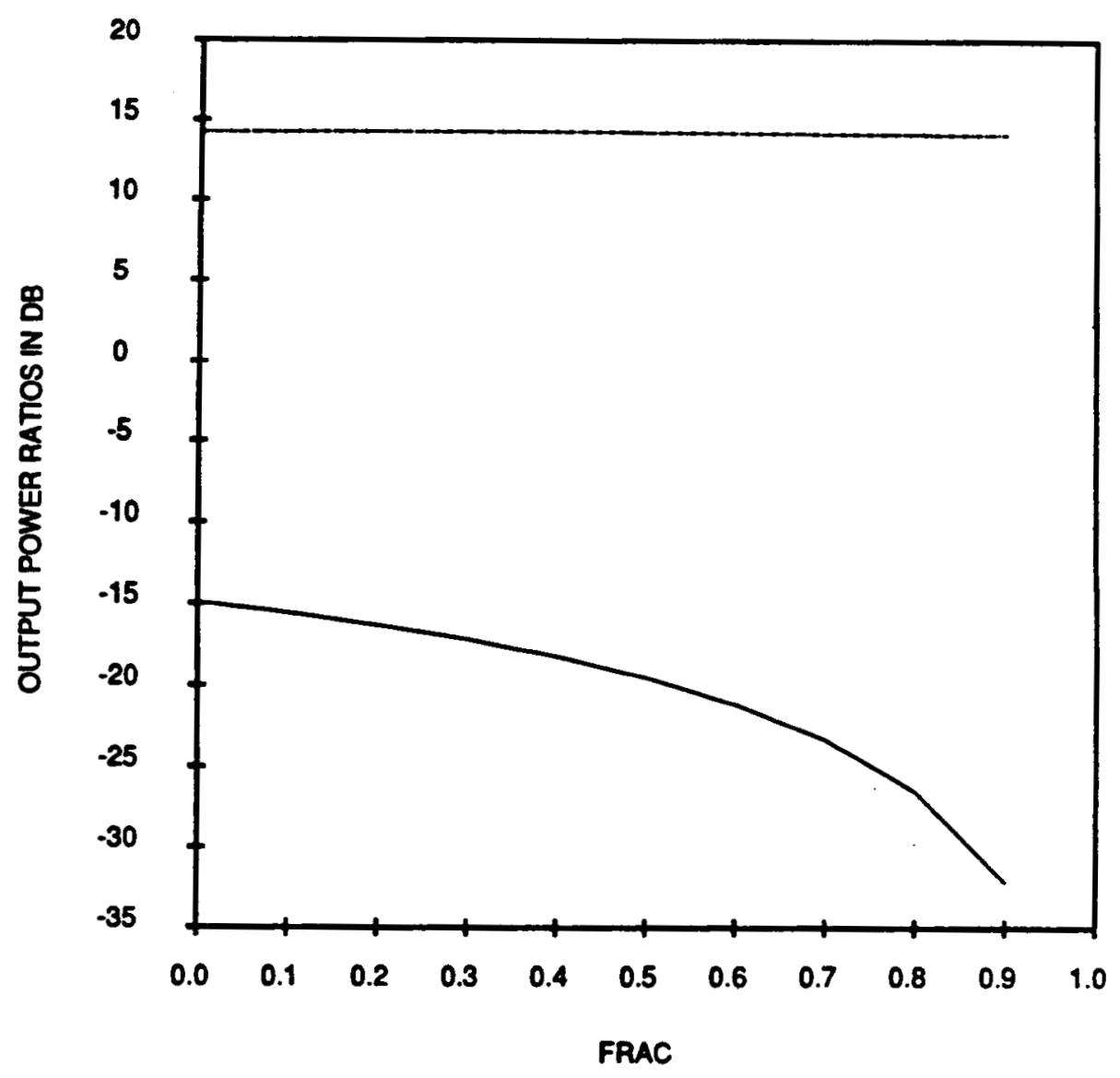

Figure 2.3: Output INR and SINR of the 4-auxiliary element adaptive array versus fraction $F$. SNR(main) $=14.6 \mathrm{~dB}, \operatorname{SNR}($ aux $)=-10 \mathrm{~dB}, \mathrm{INR}$ (main) $=-5 \mathrm{~dB}, \operatorname{INR}($ aux $)=-3 \mathrm{~dB}, \theta_{D}=0^{\circ}, \theta_{I 1}=30^{\circ}$. 
positive-definite Hermitian covariance matrix $\Phi$ be arranged in order of decreasing magnitude

$$
\lambda_{1} \geq \lambda_{2} \geq \cdots \geq \lambda_{N}>0
$$

and let $e_{i}$ be the associated orthonormal eigenvectors. The desired eigenexpansions of $\Phi$ and $\Gamma$ are

$$
\begin{aligned}
& \Phi=\sum_{n=1}^{N} e_{n} e_{n}^{H} \lambda_{n}, \text { and } \\
& \Gamma=\sum_{n=1}^{N} e_{n} e_{n}^{H}\left[\lambda_{n}-F \sigma^{2}\right]
\end{aligned}
$$

where the result $\sum_{n=1}^{N} e_{n} e_{n}^{H}=I_{N \times N}$ has been used in 2.26. The inverses needed in 2.21 and 2.23 can thus be written

$$
\begin{aligned}
\Phi^{-1} & =\sum_{n=1}^{N} \frac{e_{n} e_{n}^{H}}{\lambda_{n}} \\
\Gamma^{-1} & =\sum_{n=1}^{N} \frac{e_{n} e_{n}^{H}}{\lambda_{n}-F \sigma^{2}}
\end{aligned}
$$

where it is easily demonstrated that $\Phi \Phi^{-1}=\Gamma \Gamma^{-1}=I$ using 2.25-2.28 and the orthonormality of the $e_{i}$.

Additional observations can be made about the modified SMI weights by defining two subspaces of complex $\mathrm{N}$-dimensional space $\mathcal{C}^{N}$ as

$$
\begin{aligned}
\mathcal{S} & =s p\left[S_{D}, S_{I 1}, S_{I 2}, \ldots, S_{I M}\right] \text { and } \\
\mathcal{N} & =\mathcal{S}^{\perp}
\end{aligned}
$$

where $s p$ denotes span and $\perp$ is the orthogonal complement. Subspace $\mathcal{S}$, the span of the signal vectors, is commonly called the signal subspace, 
whereas $\mathcal{N}$ is called the noise subspace. The noise subspace has dimension $\geq 1$ since it is assumed that $N \geq M+2$. From 2.14 and the definition of eigenvectors

$$
\left[S_{D} S_{D}^{H}+\left(\sum_{m=1}^{M} S_{I m} S_{I m}^{H}\right)+\sigma^{2} I\right] e_{n}=\lambda_{n} e_{n} .
$$

for $1 \leq n \leq N$. Notice that any member of $\mathcal{N}$ is an eigenvector of $\Phi$ since for any such vector $V \in \mathcal{N}$ we have $S_{D}^{H} V=S_{m}^{H} V=0$. From here we may conclude that $e_{n} \in \mathcal{N}$ for exactly $N-M-1$ values of $n$ since if there were less, $\left\{e_{n}\right\}_{n=1}^{N}$ could not span $\mathcal{N}$ and if there were more, $\left\{e_{n}\right\}_{n=1}^{N}$ could not span $\mathcal{S}$. The assumption is made that no two signal vectors are colinear (i.e. the signals arrive from different directions). Furthermore, the eigenvalue associated with every such $e_{n}$ is the noise power $\sigma^{2}$ as seen in 2.30. For this reason, these $N-M-1$ eigenvectors are called noise eigenvectors. Hence the name noise subspace for $\mathcal{N}$.

The remaining $M+1$ eigenvectors of $\Phi$ must be in the $M+1$ dimensional subspace $\mathcal{S}$, and are referred to as the signal or principal eigenvectors. The eigenvalues associated with these principal eigenvectors are real and greater than or equal to $\sigma^{2}$. This result follows from the fact that a principal eigenvector of $\Phi$ with $\sigma^{2}>0$ is also a principal eigenvector of the same $\Phi$ with $\sigma^{2}$ set to zero. The noiseless $\Phi$ is still nonnegative definite.

Since the eigenvalues were ordered from largest to smallest in 2.24 , we have from the above argument that $\left\{e_{n}\right\}_{n=1}^{M+1}$ are the principal eigenvectors with associated eigenvalues $\lambda_{n} \geq \sigma^{2}$ and $\left\{e_{n}\right\}_{n=M+2}^{N}$ are the noise eigenvectors with associated eigenvalues $\lambda_{n}=\sigma^{2}$. 
Now substitute 2.28 in 2.23 , to see that the modified SMI weights are

$$
W=\mu \sum_{n=1}^{N}\left(\frac{e_{n}^{H} S_{D}}{\lambda_{n}-F \sigma^{2}}\right) e_{n},
$$

a linear combination of the eigenvectors of $\Phi$. In fact, since $e_{n}^{H} S_{D}=0$ for $n=M+2, M+3, \ldots, N$, the weights are ideally a linear combination of only the principal eigenvectors. In Section 3.3 we shall see the effects of using just the first $M+1$ terms of 2.31 in the SMI array computer simulation.

\subsection{Instructive Example}

To better appreciate the modification made to $\Phi$ in 2.22 lets compare the expressions for $W_{S}$ and $W$ for a particular scenario. Using 2.21, 2.23, 2.27, and 2.28 and some algebra, Gupta [1] with reference to Compton [8] showed that for the case of no desired signal and a single CW interference signal of amplitude $A$ incident on an $N$-element array, the optimal weight vectors can be written

$$
W_{S}=\frac{\mu}{\sigma^{2}}\left[S_{D}-\frac{1}{1+\frac{\sigma^{2}}{N A^{2}}} e_{1} \alpha_{1}\right]
$$

and

$$
W=\frac{\mu}{(1-F) \sigma^{2}}\left[S_{D}-\frac{1}{1+\frac{(1-F) \sigma^{2}}{N A^{2}}} e_{1} \alpha_{1}\right]
$$

where $\alpha_{1}=e_{1}^{H} S_{D}$. For a weak signal and not too many elements, $N A^{2} / \sigma^{2} \ll$ 1 so that 2.32 and 2.33 become

$$
W_{S} \approx \frac{\mu}{\sigma^{2}} S_{D}
$$

and

$$
W \approx \frac{\mu}{(1-F) \sigma^{2}}\left[S_{D}-e_{1} \alpha_{1}\right]
$$


where $F$ has been chosen such that $(1-F) \sigma^{2} /\left(N A^{2}\right) \ll 1$. It is seen from 2.34 that using the standard SMI weights $W$ simply scales the quiescent pattern of the array and thus fails to achieve the goal of adapting to the interference signal. On the other hand, for an appropriate choice of the fraction $F$, the modified weights result in an antenna pattern which does adapt to the signal scenario since $e_{1}$ contains interference signal direction information.

\subsection{Summary}

In this chapter, we have seen that the modified SMI algorithm is designed to maximize a modified SINR which leads to increased interference suppression as $F$ is increased from zero to one. The eigenstructure of $\Phi$, which was analyzed in detail, has led to a useful geometric interpretation of the SMI algorithm and has prompted us to investigate in the next chapter the effects of omitting the noise eigenvectors from the weight expression of 2.31 .

Up to this point, knowledge of the true covariance matrix $\Phi$ has been assumed. In practice, however, $\Phi$ must be estimated by an average involving a finite number of signal samples. The next chapter addresses through theory and simulation a number of topics concerning the performance of the modified SMI algorithm when only estimates of $\Phi$ are available. 


\section{Chapter 3}

\section{Modified SMI Array Performance with Estimated Covariance}

This chapter studies the effects of covariance matrix estimation on the performance of the modified SMI antenna array. The first section simply introduces the particular K-snapshot-based covariance estimate $\hat{\Phi}_{K}$ used in this study. The next section presents a theoretical statistical analysis of modified SMI array performance using the estimate $\hat{\Phi}_{K}$ in the place of $\Phi$ in 2.22. Much of this analysis will be independent of the assumed signal model. Only at the end of the analysis is a particular signal model (corresponding to our satellite communication application) assumed.

Computer code has been developed which simulates a real modified SMI array operating in the satellite signal environment and, in addition, implements the results of the statistical analysis for comparison purposes. The signal model used in the computer simulation code is described. Simulations are then used to discuss the estimation of $\sigma^{2}$, to verify the statistical 
theory and comment on the choice of the fraction F, to study the effects of omitting the noise eigenvectors from the weight estimate (as proposed at the end of Section 2.3), and finally to characterize the types of error in the estimated covariance matrix.

\subsection{Sample Covariance Matrix}

In a real system, the true covariance matrix is unknown and must be estimated by averaging a number of signal snapshot outer products. A snapshot $X_{k}$ is an N-vector of samples resulting from a simultaneous sampling of the $\mathrm{N}$ antenna element signals. The estimate of the true covariance matrix is called the sample covariance matrix. In particular, the maximum likelihood estimate ([9], Theorem 4.1)

$$
\hat{\Phi}_{K}=\frac{1}{K} \sum_{k=1}^{K} X_{k} X_{k}^{H}
$$

of $\Phi$ given K snapshots and no knowledge of the signal environment is used here.

\subsection{Statistical Analysis Results}

Ganz, Moses, and Wilson [7] have provided a statistical analysis of the modified SMI weight and power estimators assuming that the true noise power $\sigma^{2}$, true steering vector $S_{D}$, and sample covariance matrix $\hat{\Phi}_{K}$ are used in 2.22. The results of the analysis are presented here. It is explained how one can apply much of this work to any signal scenario including wideband signals. Specific results have been obtained for a narrowband 
(sinusoidal) scenario consisting of one desired signal and $M$ interference signals arriving from arbitrary directions at an equi-spaced linear array of elements of arbitrary gain and spacing. The notation introduced in Sections 2.1 and 3.1 is used.

The statistical results consist of bias and variance expressions which describe the convergence properties of the array weights and output signal powers as a function the number of snapshots $K$, the choice of fraction $F$, and the signal scenario. The expressions have been implemented in the computer simulation so that curves representing expected value and confidence intervals might overlay "trial runs" of the simulated array. Agreement between the statistical curves and the trial runs would build confidence in the derivation and implementation of the statistical curves as well as in the implementation of the array simulator. In fact, the statistical curves characterize the transient performance of the array and thus may be used in place of expensive, time-consuming Monte Carlo simulations. Furthermore, it is hoped that the statistical curves might act as a standard with which other weight adaptation schemes may be compared.

It is desired to analyze the performance of the modified SMI array whose weights are based on $K$ snapshots, thus this presentation begins with 2.22 restated below. The optimal modified SMI weight vector $W$ and its Ksnapshot-based estimate $\hat{W}_{K}$ are

$$
\begin{aligned}
W & =\mu\left[\Phi-F \sigma^{2} I\right]^{-1} S_{D} \\
& =\mu[\Gamma]^{-1} S_{D}
\end{aligned}
$$


and

$$
\begin{aligned}
\hat{W}_{K} & =\mu\left[\hat{\Phi}_{K}-F \sigma^{2} I\right]^{-1} S_{D} \\
& =\mu\left[\hat{\Gamma}_{K}\right]^{-1} S_{D}
\end{aligned}
$$

where $\Gamma \equiv \Phi-F \sigma^{2} I$ and $\hat{\Gamma}_{K} \equiv \hat{\Phi}_{K}-F \sigma^{2} I$ are the optimal and estimated values of the modified covariance matrix and $\hat{\Phi}_{K}$ is given by 3.1 . Define the error $\tilde{W}_{K}$ in the K-snapshot-based weight estimate and modified covariance estimate error $\tilde{\Gamma}_{K}$ by

$$
\begin{aligned}
\tilde{W}_{K} & \equiv W-\hat{W}_{K} \\
& =\mu\left[\Gamma^{-1}-\hat{\Gamma}_{K}^{-1}\right] S_{D}
\end{aligned}
$$

and

$$
\begin{aligned}
\tilde{\Gamma}_{K} & \equiv \Gamma-\hat{\Gamma}_{K} \\
& =\Phi-\hat{\Phi}_{K} \\
& \equiv \tilde{\Phi}_{K} .
\end{aligned}
$$

The error in the modified and unmodified covariance is the same. The expected value and variance of the $K$-snapshot-based weights can easily be expressed as

$$
\begin{aligned}
E[\hat{W}] & =W-E[\tilde{W}] \\
\operatorname{var}[\hat{W}] & \equiv E\left[(\hat{W}-E[\hat{W}])(\hat{W}-E[\hat{W}])^{H}\right] \\
& =\operatorname{var}[\tilde{W}] \\
& =E\left[\tilde{W} \tilde{W}^{H}\right]-E[\tilde{W}] E[\tilde{W}]^{H}
\end{aligned}
$$


where the subscripts ${ }_{K}$ have been omitted for simplicity. The output signal powers of an array given a set of $\mathrm{K}$-snapshot-based weights are

$$
\begin{aligned}
\hat{P}_{J} & =E\left[\left|\hat{s}_{J}(t)\right|^{2}\right] \\
& =E\left[\hat{W}^{H} X_{J} X_{J}^{H} \hat{W}\right] \\
& =\hat{W}^{H} E\left[X_{J} X_{J}^{H}\right] \hat{W} \\
& =\hat{W}^{H} \Phi_{J} \hat{W}
\end{aligned}
$$

where $J=D, I m$, or $\eta$, for desired, $m^{\text {th }}$ interference, or noise power, respectively. The expected value and variance of the output signal powers are

$$
\begin{aligned}
E\left[\hat{P}_{J}\right] & =E\left[\hat{W}^{H} \Phi_{J} \hat{W}\right] \\
& =E\left[(W-\tilde{W})^{H} \Phi_{J}(W-\tilde{W})\right] \\
& =P_{J}-E[\tilde{W}]^{H} \Phi_{J} W-W^{H} \Phi_{J} E[\tilde{W}]+E\left[\tilde{W}^{H} \Phi_{J} \tilde{W}\right]
\end{aligned}
$$

and

$$
\begin{aligned}
\operatorname{var}\left[\hat{P}_{J}\right]= & E\left[\left(\hat{P}_{J}-E\left[\hat{P}_{J}\right]\right)^{2}\right] \\
= & E\left[\left(\tilde{W}^{H} \Phi_{J} \tilde{W}\right)^{2}\right]-\left(E\left[\tilde{W}^{H} \Phi_{J} \tilde{W}\right]\right)^{2} \\
& -4 \operatorname{Re}\left\{E\left[\tilde{W}^{H} \Phi_{J} \tilde{W} \tilde{W}^{H} \Phi_{J} W\right]\right\} \\
& \quad+4 \operatorname{Re}\left\{E\left[\tilde{W}^{H} \Phi_{J} \tilde{W}\right] E[\tilde{W}]^{H} \Phi_{J} W\right\} \\
& +4 E\left[\left(\operatorname{Re}\left\{\tilde{W}^{H} \Phi_{J} W\right\}\right)^{2}\right]-4\left(\operatorname{Re}\left\{E[\tilde{W}]^{H} \Phi_{J} W\right\}\right)^{2}
\end{aligned}
$$

where $R e$ denotes real part and $J$ is as before. Note that 3.6, 3.7, 3.9, and 3.10 express the desired quantities in terms of the statistics of $\bar{W}$ and that $\tilde{W}$ is a function of $\hat{\Phi}_{K}$, an average of $\mathrm{K}(N \times N)$ random variables. 
By the Central Limit Theorem, $\tilde{W}$ approaches a Gaussian random variable for reasonably large $\mathrm{K}$. Under the Gaussian assumption, the higher order statistics of $\tilde{W}$ needed for the first and third terms of 3.10 may be found [6] in terms of its first and second order statistics.

The problem has now been reduced to finding $E[\tilde{W}], E\left[\tilde{W} \tilde{W}^{H}\right]$, and $E\left[\tilde{W} \bar{W}^{T}\right]$. Evaluation of these expectations is accomplished by first manipulating $\hat{\Gamma}^{-1}$ using 3.5

$$
\begin{aligned}
\hat{\Gamma}^{-1} & =[\Gamma-\tilde{\Gamma}]^{-1} \\
& =\left[\left(I-\tilde{\Gamma} \Gamma^{-1}\right) \Gamma\right]^{-1} \\
& =\Gamma^{-1}\left(I-\tilde{\Gamma} \Gamma^{-1}\right)^{-1}
\end{aligned}
$$

Now rewrite 3.4 by factoring $\Gamma^{-1}$ to the front, substituting for $\hat{\Gamma}^{-1}$ using 3.11 , and expanding $\left[I-\tilde{\Gamma} \Gamma^{-1}\right]$ as a power series [5]. The resulting weight error may be written

$$
\tilde{W}=\mu \Gamma^{-1}\left[I-\left\{I+\tilde{\Gamma} \Gamma^{-1}+\left(\tilde{\Gamma} \Gamma^{-1}\right)^{2}+\cdots\right\}\right] S_{D}
$$

The desired expectations are

$$
\begin{aligned}
E[\tilde{W}] & \approx-\Gamma^{-1} E\left[\tilde{\Gamma} \Gamma^{-1} \tilde{\Gamma}\right] W \\
E\left[\tilde{W} \tilde{W}^{H}\right] & \approx \Gamma^{-1} E\left[(\tilde{\Gamma} W)(\tilde{\Gamma} W)^{H}\right]\left(\Gamma^{-1}\right)^{H}, \text { and } \\
E\left[\tilde{W} \tilde{W}^{T}\right] & \approx \Gamma^{-1} E\left[(\tilde{\Gamma} W)(\tilde{\Gamma} W)^{T}\right]\left(\Gamma^{-1}\right)^{T}
\end{aligned}
$$

where $E[\tilde{\Gamma}]=0$ and $W=\mu \Gamma^{-1} S_{D}$ have been used. The approximations result from neglecting terms involving higher powers of $\tilde{\Gamma}$. The approximations are justified at the end of this section. Using 3.5 , it is easy to write 
3.13-3.15 in terms of $E\left[\tilde{\Phi}_{i l} \tilde{\Phi}_{s t}^{*}\right]$, the expected value of the product of the $i t^{\text {th }}$ and $s t^{\text {th }}$ elements of the $\mathrm{K}$-snapshot-based covariance error matrix.

The statistical results presented to this point have been very general in that they have been derived independent of any assumed signal scenario. Only now, for the calculation of $E\left[\tilde{\Phi}_{i l} \tilde{\Phi}_{a t}^{*}\right]$, must a scenario be assumed. In order to apply this work to different signal scenarios one evaluates $E\left[\tilde{\Phi}_{i l} \tilde{\Phi}_{s t}^{*}\right]$ for the scenario of interest. The power series expansion of 3.12 and the approximations of 3.13-3.15 are valid for any signal scenario when $\mathrm{K}$ is large enough.

For the case of one sinusoidal desired signal, and $M$ sinusoidal interference signals arriving from arbitrary directions at an $\mathrm{N}$-element equi-spaced linear array with complex Gaussian noise, $N\left(0, \sigma^{2}\right)$, at each element, the expectation is $[7]$

$$
\begin{aligned}
E\left[\tilde{\Phi}_{i l} \tilde{\Phi}_{s t}^{*}\right]= & \frac{1}{K}\left[\sigma^{4} \delta_{t l} \delta_{i s}\right. \\
& +\sum_{\alpha=1}^{M} \sum_{\substack{s=1 \\
\alpha \neq \beta}}^{M} a_{I \alpha i} a_{I \alpha s} e^{-j(i-s) \phi_{I \alpha}} a_{I \beta l} a_{I \beta t} e^{-j(t-l) \phi_{I \beta}} \\
& +\sum_{\alpha=1}^{M}\left\{a_{D i} a_{D s} e^{-j(i-s) \phi_{D}} a_{I \alpha l} a_{I \alpha t} e^{-j(t-l) \phi_{I \alpha}}\right. \\
& \left.+a_{D l} a_{D t} e^{-j(t-l) \phi_{D}} a_{I \alpha i} a_{I \alpha s} e^{-j(i-s) \phi_{I \alpha}}\right\} \\
+ & \sum_{\alpha=1}^{M} \sigma^{2}\left\{\delta_{t l} a_{I \alpha i} a_{I \alpha s} e^{-j(i-s) \phi_{I \alpha}}+\delta_{i s} a_{I \alpha l} a_{I \alpha t} e^{-j(t-l) \phi_{I \alpha}}\right\} \\
& \left.+\sigma^{2}\left\{\delta_{t l} a_{D i} a_{D s} e^{-j(i-s) \phi_{D}}+\delta_{i s} a_{D l} a_{D t} e^{-j(t-l) \phi_{D}}\right\}\right]
\end{aligned}
$$

where $\delta_{t l}$ is the Kronecker delta. Note that $E\left[\tilde{\Phi}_{i l} \tilde{\Phi}_{t s}\right]=E\left[\tilde{\Phi}_{i l} \tilde{\Phi}_{s t}^{*}\right]$ since $\Phi$ and $\hat{\Phi}_{K}$ are Hermitian symmetric. 
The justification for the approximations used in 3.13-3.15 is now apparent. Expectations involving higher powers (3 or greater) of $\tilde{\Gamma}$ require higher order statistics of $\tilde{\Phi}$. By the Central Limit Theorem, the elements of $\tilde{\Phi}$ are approximately Gaussian for $\mathrm{K}$ large enough. By 3.16 , the $E\left[\tilde{\Phi}_{i l} \tilde{\Phi}_{s t}^{*}\right]$ are $O(1 / K)$. Using familiar expressions [6] for higher order statistics of Gaussian random variables it follows that such terms are $o(1 / K)$ and thus are justifiably neglected for $\mathrm{K}$ large.

It should be mentioned that of all six terms in 3.10 for $\operatorname{var}\left[\hat{P}_{J}\right]$ only the fifth is proportional to $(1 / K)$ whereas each of the others has $O\left(1 / K^{2}\right)$ contributions. It was found necessary to include in the computer code the $O\left(1 / K^{2}\right)$ terms of 3.10 (in addition to the fifth term) in order to yield accurate confidence intervals for the $\mathrm{K}$ values of interest.

In summary, the estimated weights and resultant output powers are asymptotically unbiased and consistent. The biases of the weight and power estimators decrease at a rate proportional to $(1 / K)$ while the asymptotic standard deviations decrease at a rate proportional to $(1 / \sqrt{K})$.

\subsection{Computer Simulation and Observations}

\subsubsection{Signal Snapshot Model}

The simplified model of the signal snapshot $X_{k}$ of 3.1 used in the computer simulation reflects the satellite/earth station communication link application. A series of snapshots is not simulated by evaluating the signal vector of 2.1 at equally-spaced times since the time between snapshots can vary in a real array. To account for this, the phases of signals have been decor- 
related from snapshot to snapshot resulting in a model of the form

$$
X_{k}=X_{D k}+X_{\eta k}+\sum_{m=1}^{M} X_{I m k}
$$

for the case of $M$ interference signals, where

$$
X_{J k}=A_{J} U_{J} \exp \left(j \beta_{J k}\right) \quad J=D, I 1, I 2, \ldots, \text { or } I M
$$

and

$$
X_{\eta k}=\left[\begin{array}{llll}
\eta_{1 k} & \eta_{2 k} & \cdots & \eta_{N k}
\end{array}\right]^{T} .
$$

The $A_{J}$ and $U_{J}$ were defined in 2.7 and 2.8 , the $\beta_{J k}$ are uniformly distributed random variables on the interval $[0,2 \pi]$, and the $\eta_{n k}$ are complex zeromean Gaussian random variables of variance $\sigma^{2}$. All random variables are mutually independent. The steering vector $S_{D}=A_{D} U_{D}$ is assumed to be known exactly.

The estimated modified weights based on $\mathrm{K}$ snapshots are

$$
\begin{aligned}
\hat{W}_{K} & =\mu\left[\hat{\Phi}_{K}-F \sigma^{2} I\right]^{-1} S_{D} \\
& =\mu\left[\hat{\Gamma}_{K}\right]^{-1} S_{D}
\end{aligned}
$$

from 2.22 with the covariance estimate $\hat{\Phi}_{K}$ of 3.1 replacing the true covariance $\Phi$. The constant $\mu$ of 2.22 is chosen as unity. Note that the constant $\mu$ simply scales the weights and powers by $\mu$ and $\mu^{2}$, respectively, and has no effect on power ratios such as the MSINR of 2.20. Since the Gaussian noise random variables $\eta_{n k}$ are computer generated, their true variance $\sigma^{2}$ is known to the programmer and will be used, at first, in implementing 3.20. Subsequently, $\sigma^{2}$ will be estimated by the minimum eigenvalue $\hat{\sigma}^{2}=\hat{\lambda}_{N}$ of $\hat{\boldsymbol{\Phi}}_{K}$ in order to more realistically model an actual SMI antenna array. 


\subsubsection{Interpreting the Plots}

The modified SMI algorithm has been implemented on a VAX 11-785 computer using the above snapshot model and statistical analysis results. The code yields plots of the weights, output powers and power ratios, and sample covariance matrix eigenvalues versus the number of snapshots used in the covariance estimate for a particular fraction $F$. Specifically, a typical plot consists of

1. a number of "trial runs" of the simulated array which appear as jagged lines,

2. a straight horizontal line giving the value (as found in Section 2.2) of the performance measure assuming the true covariance is known,

3. the expected value of the estimator which is a smooth curve that lies among the trial runs and asymptotically approaches the true covariance value, and

4. two smooth curves (one above and one below the expected value curve) determined from the estimator variance that represent a $95 \%$ confidence interval ( \pm 2 standard deviations) for the estimator.

See Figure 3.13 for a graph whose curves have been fully labeled.

Some further comments to aid in the understanding of the following plots are in order. First, items 3 and 4 above appear only on Figures 3.2-3.15 since the simulations shown on these figures are ones to which the theory of Section 3.2 applies. Second, graphs of SINR and INR do not include 
items 3 and 4 above since expressions for bias and variance of power ratios are still being developed. Third, the "lower" variance curve sometimes does not appear on graphs of supressed interference power (Figure 3.10, for example) simply because the "warping" due to plotting in $\mathrm{dB}$ causes the curve to lie outside the range of the graph. Fourth, some graphs of desired signal power (for example, Figure 3.6) show confidence intervals that exhibit unusual behavior for small numbers of snapshots $K$. This behavior occurs in $\mathrm{K}$-regions where the power variance estimate is negative. To account for negative variance estimates, recall that higher powers of $\tilde{\Gamma}_{K}$ were neglected in Equations 3.13-3.15 of the statistical analysis. Since $\left\|\tilde{\Gamma}_{K}\right\| \rightarrow 0$ with probability 1 as $K \rightarrow \infty$, neglecting these terms was equivalent to making a large $\mathrm{K}$ assumption. Furthermore, the number which is being estimated, the desired signal power variance, is itself a relatively small positive number as evidenced by the trial runs and the scale of the desired signal power graphs. For these reasons, the "unusual" behavior is not at all surprising. Finally, in several graphs (for example Figure 3.11) the scale of the graph is such that the curves are indistinguishably close. These "poorly"-scaled graphs are a result of the authors' desire to keep the scale of comparable graphs the same in order to simplify comparisons.

Keep in mind that any consistency or inconsistency between the trial runs and the statistical curves simply comments on the validity of the statistical derivations and perhaps the quality of the random variables generated in the trials. The plots may not accurately reflect how well the above statistical snapshot model represents the situation in a real antenna array. The investigation that follows is based on the same signal scenario and array 
geometry introduced in Section 2.2.

\subsubsection{Estimating the Noise Power}

The statistical results of the previous section were derived assuming the true noise power $\sigma^{2}$ were known. Therefore, to be precise, the statistical curves should overlay simulations only when the true $\sigma^{2}$ (known to the programmer) is used in 3.20. Using the true $\sigma^{2}$ could be numerically hazardous if it is the case that $F \sigma^{2}$ is very close to $\hat{\lambda}_{N}$, the minimum eigenvalue of $\hat{\Phi}$, since then $\hat{\Gamma}$ of 3.20 would approach singularity as is seen in 2.28 with the eigen-decomposition of $\hat{\Gamma}$ replacing that of $\Gamma$.

Figure 3.1 is included in order to verify that the following simulations which use the true noise variance $\sigma^{2}$ rather than $\hat{\lambda}_{N}$ in 3.20 are numerically sound. Six trials runs were made with unity noise variance. The minimum eigenvalue $\hat{\lambda}_{N}$ of $\hat{\Phi}_{K}$ is plotted as the number of snapshots $\mathrm{K}$ is increased. The figure shows that using the true $\sigma^{2}$ in 3.20 is numerically sound for $F \leq 0.9$ and $K \geq 1500$. In fact, it has been found that it makes very little difference in the simulation results whether true or estimated noise variance is used in 3.20 since $\hat{\lambda}_{K}$ is a "good" estimate of $\sigma^{2}$ for $K$ and $F$ values in the regions of interest. Thus, we can proceed with the understanding that, in these regions of interest, the statistical theory developed in the previous section applies independent of whether true or estimated noise variance is used in the weight estimate equation 3.20 . 


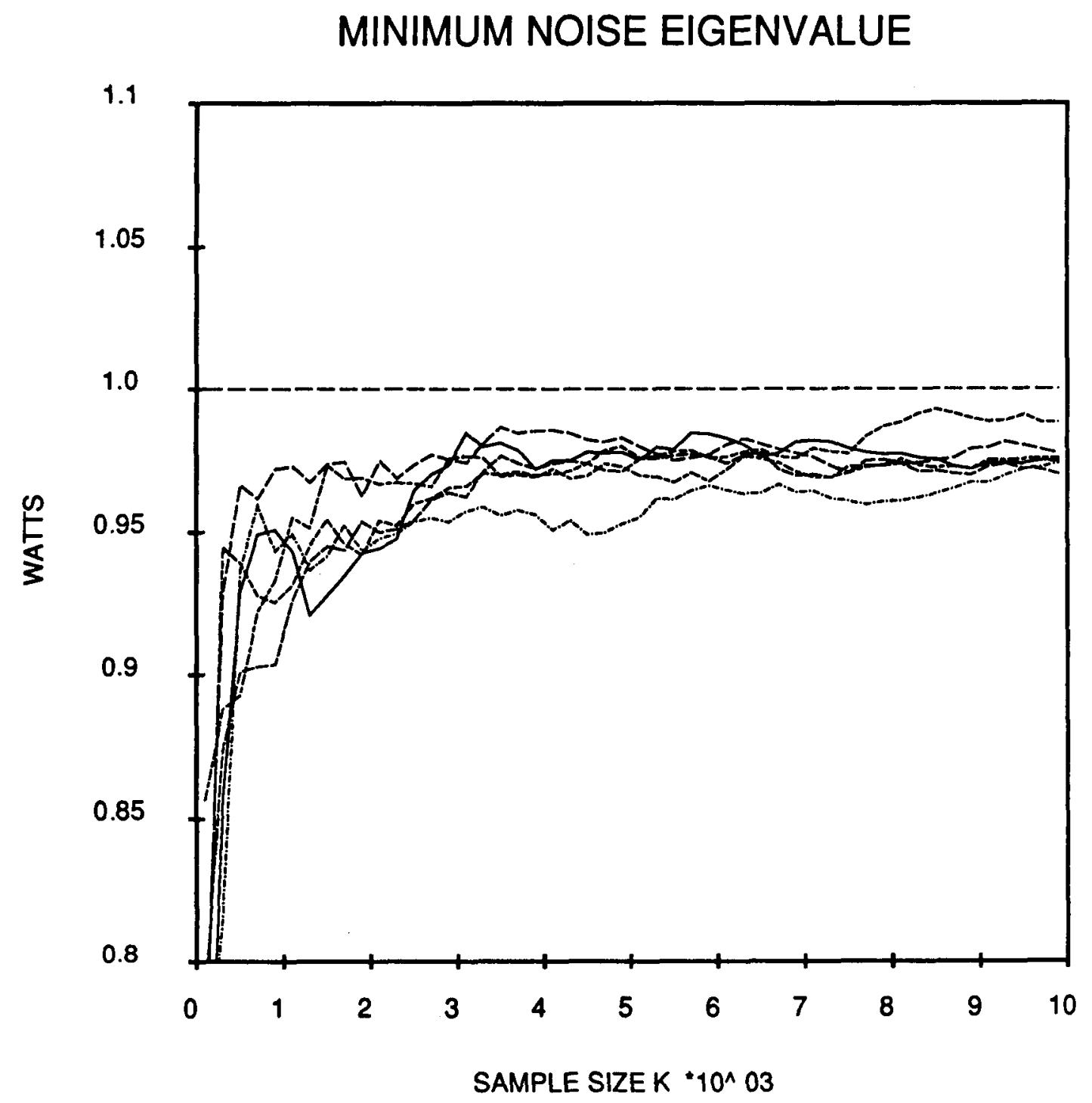

Figure 3.1: Six trials showing the minimum eigenvalue of $\hat{\Phi}_{K}$ approaching the true noise variance $\sigma^{2}=1$ as the number of snapshots $K$ in the covariance estimate increases. 


\subsubsection{Varying the Fraction $F$}

The scenario of Section 2.2 is used in all that follows. Let us begin by observing the performance of the modified algorithm for different values $F$. Figures 3.2, 3.3, and 3.4 are plots of the output INR and SINR where $F=0, F=0.8$, and $F=0.9$, respectively. Four typical trial runs were made for each value of $F$. The same set of noise seeds were used for each plot for purposes of comparison. Remember that $F=0$ corresponds to standard SMI. These figures demonstrate the degradation in the SINR as $F$ is increased. They also suggest that the increased interference suppression comes at the price of having to increase the number of snapshots in the covariance estimate to achieve that suppression. For example, comparing 3.2 and 3.3 we see that setting $F=0.8$ increases interference suppression by about $12 \mathrm{~dB}$ compared to standard SMI although it takes approximately 30,000 more snapshots to get that additional suppression. For an application in which the signal environment changes sufficiently fast, increasing $K$ may not be practical. In the application considered here, however, it may very well be practical.

Rather than look at power ratios let us back up and look at the powers themselves in order to see where the problem lies. Figures 3.5-3.7 show the desired signal power $P_{D}$ of 3.8 for $F=0, F=0.8$, and $F=0.9$, respectively. Similarly, Figures 3.8-3.10 and Figures 3.11-3.13 show the interference powers and noise powers, respectively. The statistical bias and $95 \%$ confidence intervals resulting from the statistical analysis of the previous section overlay the four trial runs and the infinite snapshot curve. 


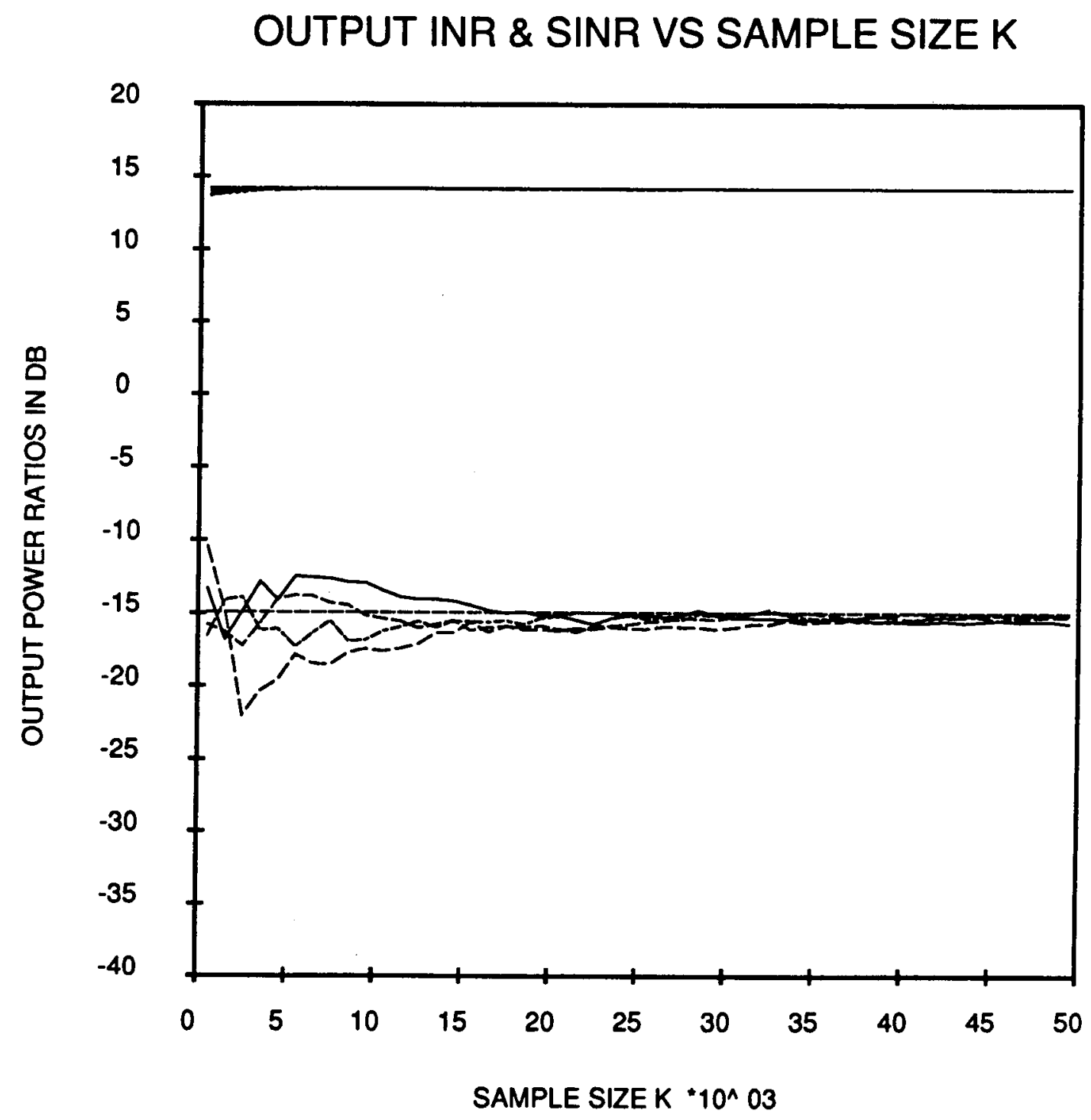

Figure 3.2: Plot of output INR and SINR versus number of snapshots $K$ for $F=0$ shows 4 trials and straight line representing the infinite data (true covariance) case. 


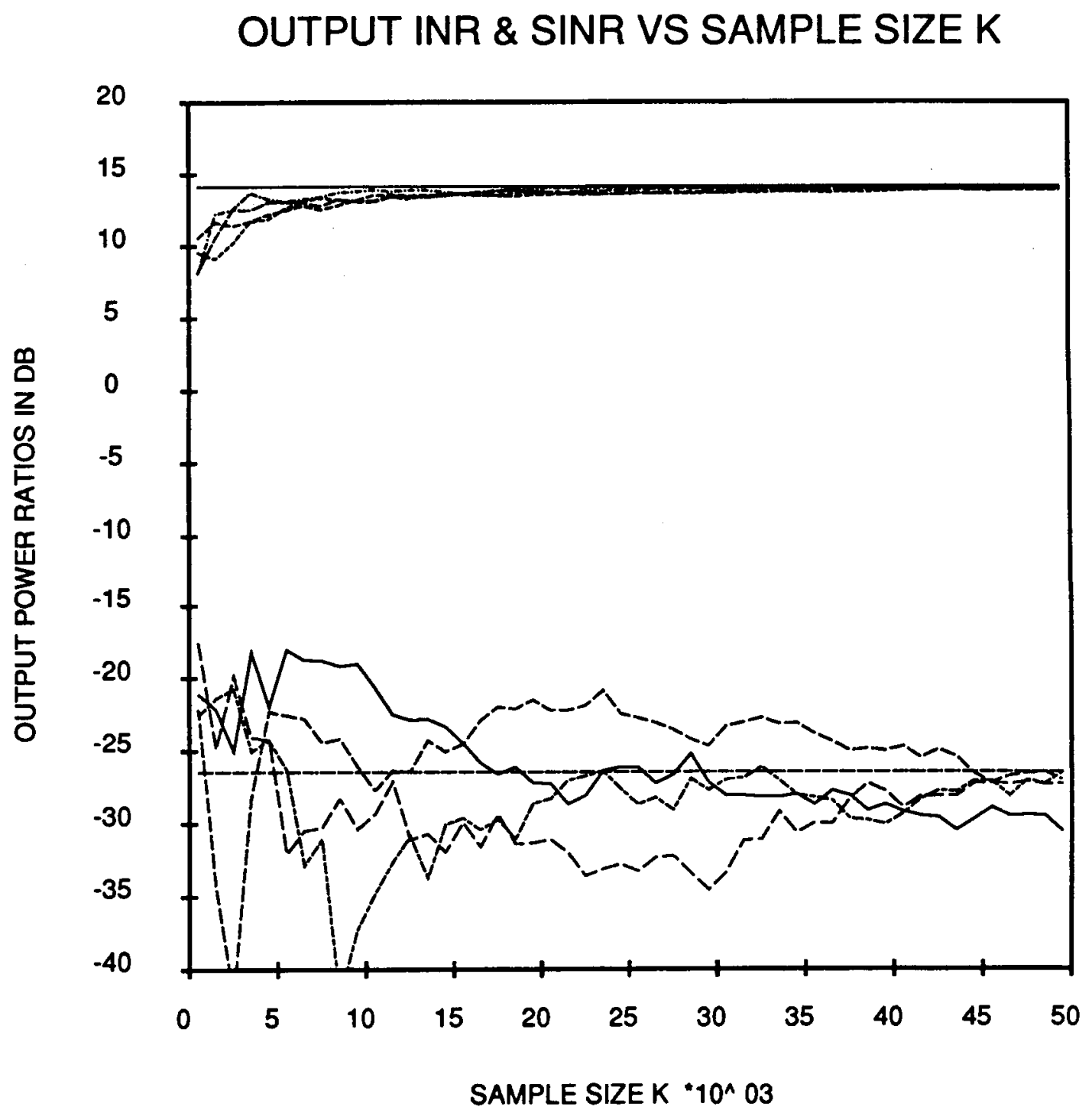

Figure 3.3: Plot of output INR and SINR versus number of snapshots $K$ for $F=0.8$ shows 4 trials and straight line representing the infinite data (true covariance) case. 


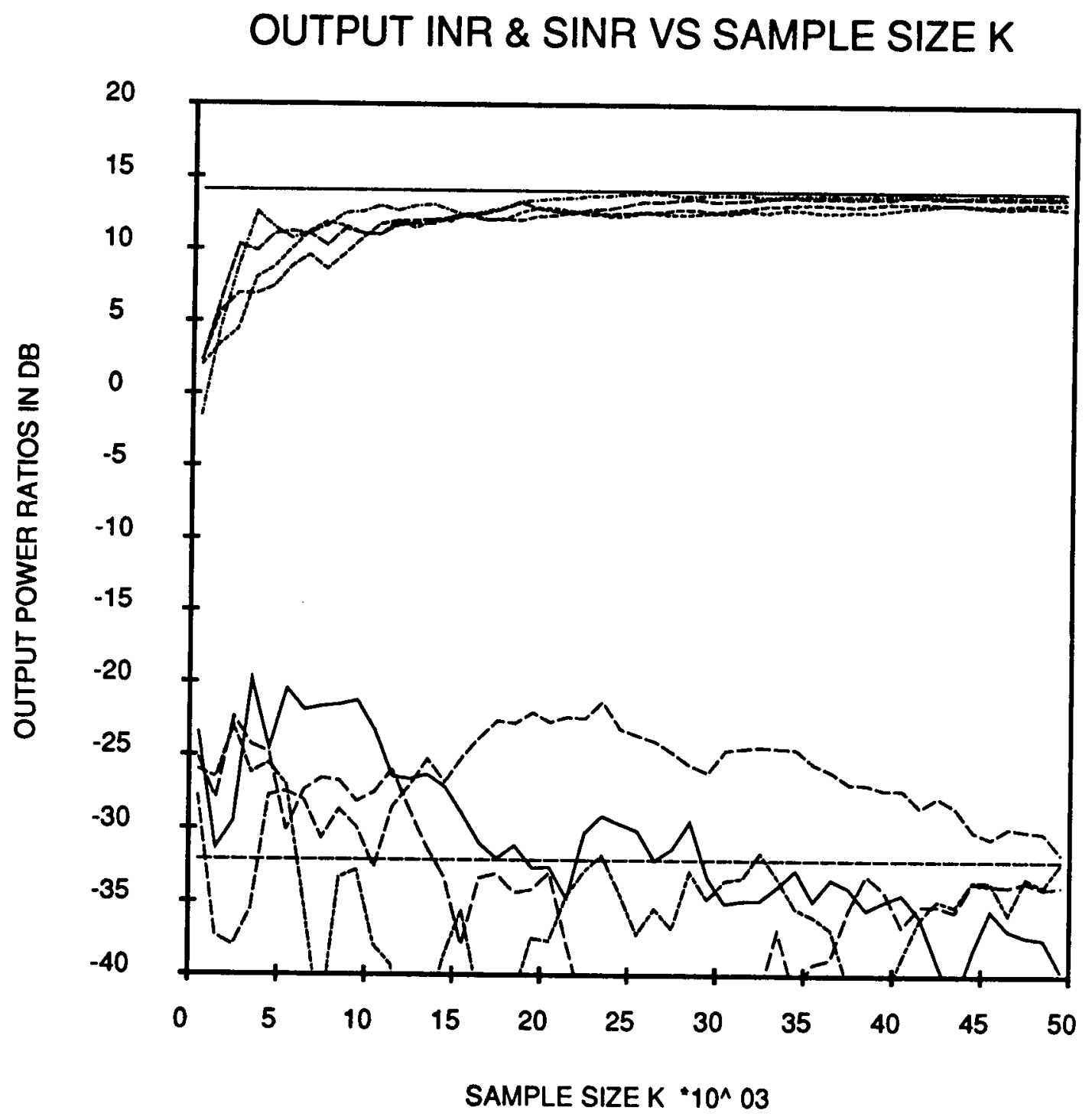

Figure 3.4: Plot of output INR and SINR versus number of snapshots $K$ for $F=0.9$ shows 4 trials and straight line representing the infinite data (true covariance) case. 


\section{OUTPUT SIGNAL POWER VS SAMPLE SIZE K}

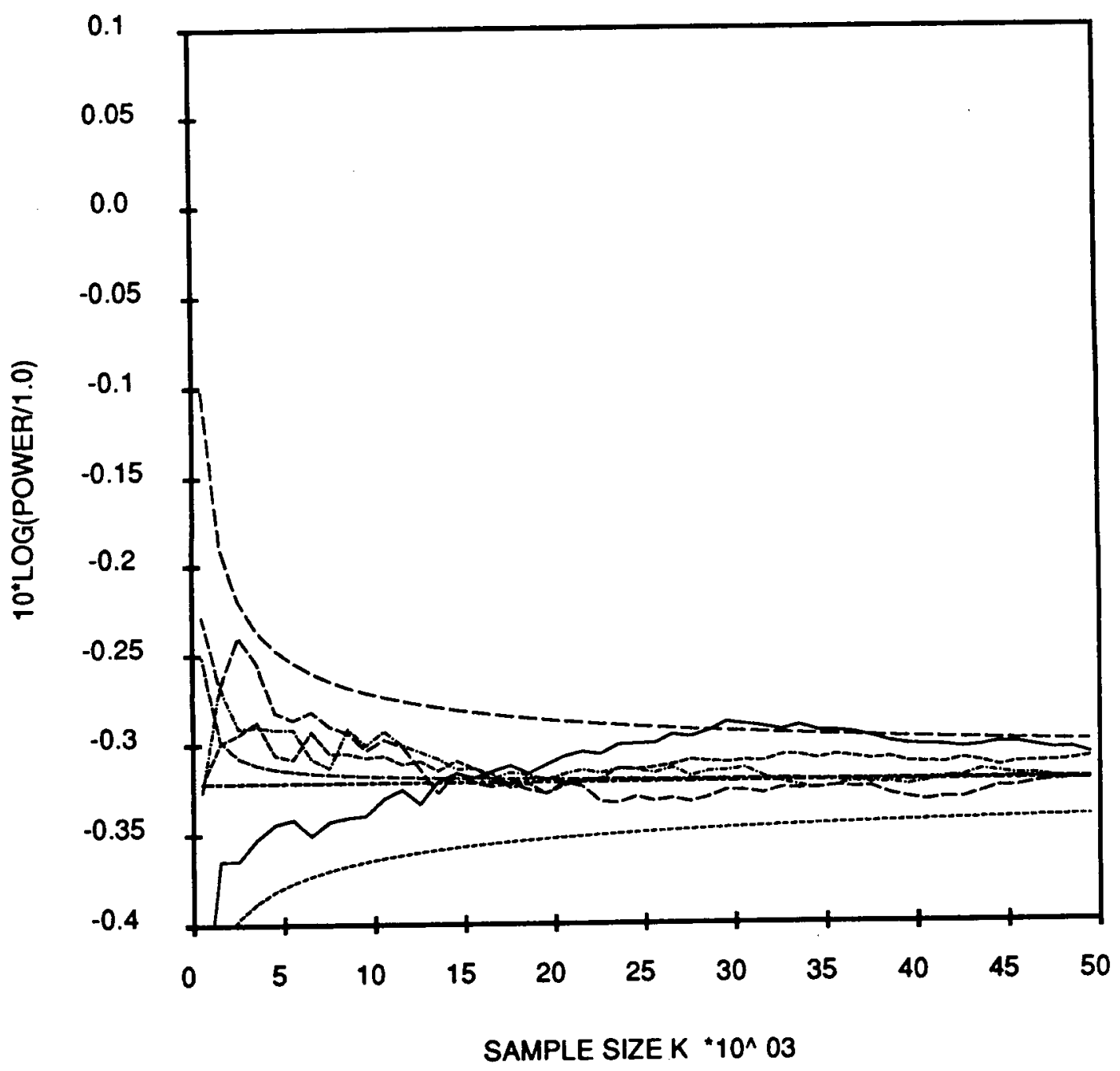

Figure 3.5: Plot of output desired signal power $P_{D}$ versus number of snapshots $K$ for $F=0.0$ shows 4 trials, true covariance curve, bias curve, and $95 \%$ confidence interval. 


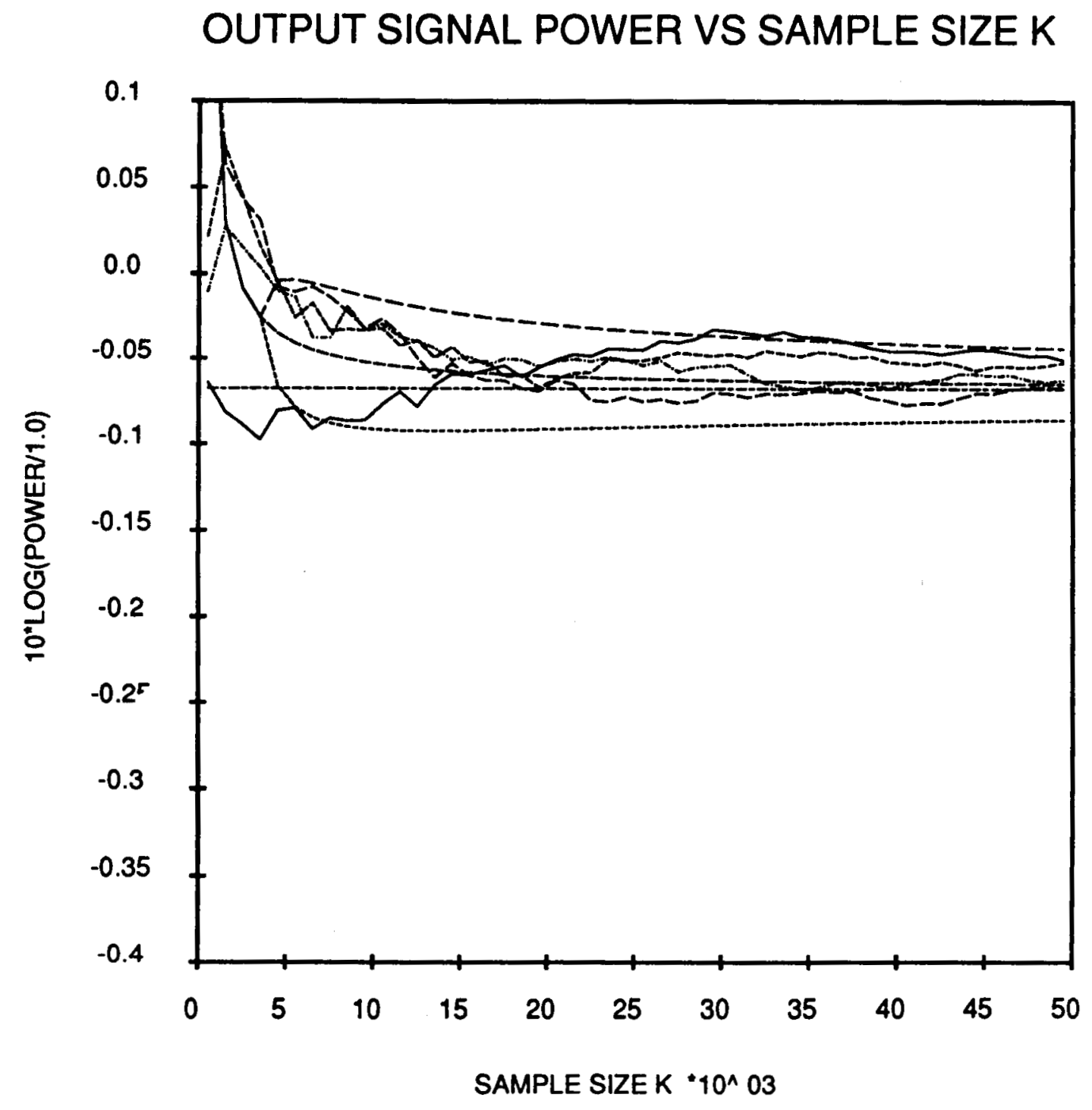

Figure 3.6: Plot of output desired signal power $P_{\mathcal{D}}$ versus number of snapshots $K$ for $F=0.8$ shows 4 trials, true covariance curve, bias curve, and $95 \%$ confidence interval. 


\section{OUTPUT SIGNAL POWER VS SAMPLE SIZE K}

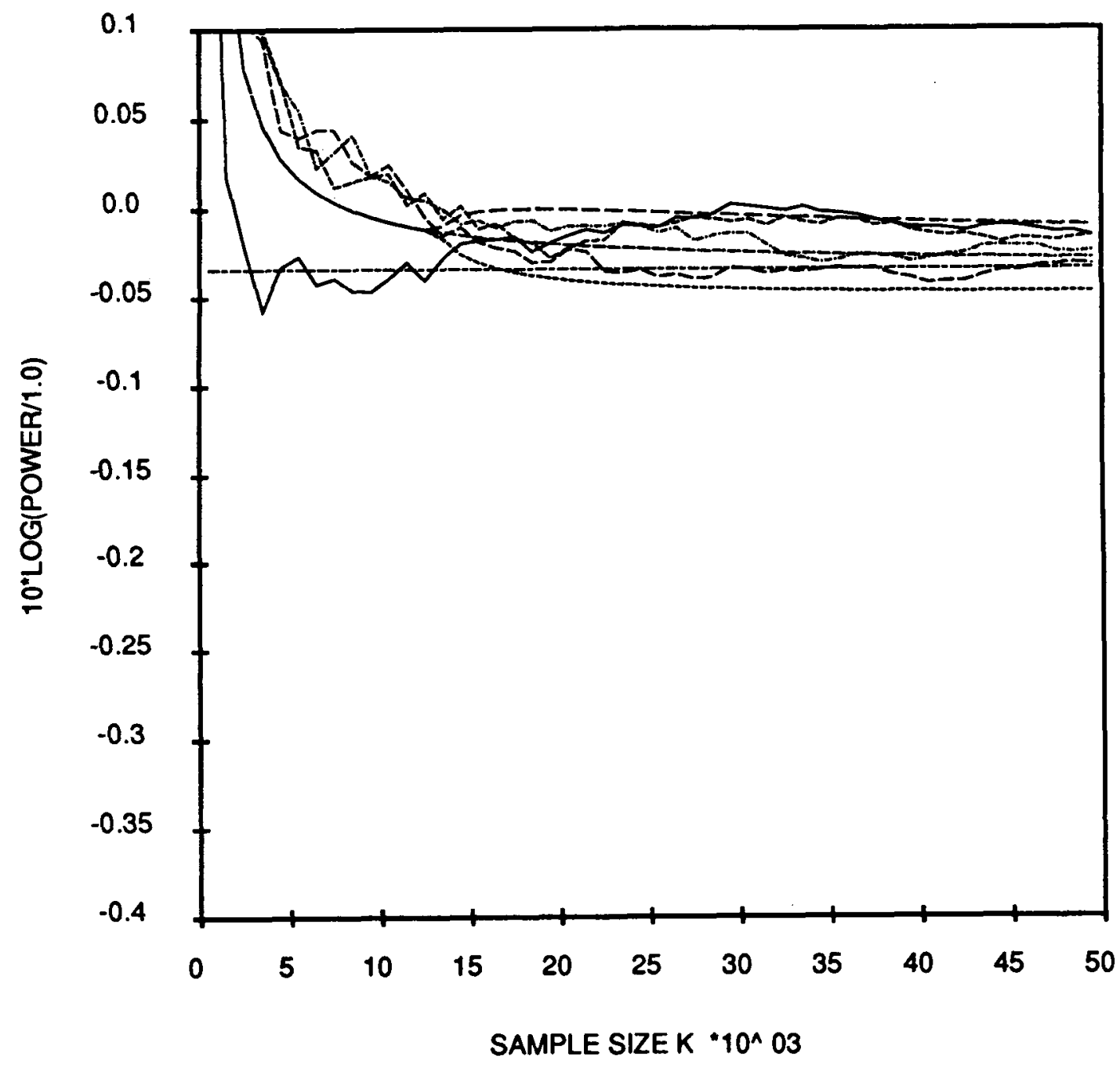

Figure 3.7: Plot of output desired signal power $P_{D}$ versus number of snapshots $K$ for $F=0.9$ shows 4 trials, true covariance curve, bias curve, and $95 \%$ confidence interval. 
In all cases the statistical curves and the trial runs seem to agree rather well. The plots show that the bias and variance of the output powers tend to increase with the fraction $F$. The inaccuracy of the desired signal power variance curves for small $\mathrm{K}$ in Figures 3.6 and 3.7 are probably explained by the neglect of $o(1 / K)$ terms in 3.13-3.15 and $o\left(1 / K^{2}\right)$ terms in 3.10.

That the inaccuracy should be apparent only in the desired signal power is not surprising since based on the trials runs this variance is much smaller than those for the interference and noise powers causing inaccuracies to be more evident.

The outstanding feature of this group of plots is the comparatively large bias and variance of the interference signal power. Specifically, for $F=0.9$, after 50,000 snapshots the difference between the upper bound of the confidence interval and the infinite snapshot interference level is about $7.5 \mathrm{db}$ whereas it is only $1.25 \mathrm{~dB}$ and $0.03 \mathrm{~dB}$ for noise and desired signal powers, respectively. The explanation is intuitive from an array pattern viewpoint. Since the modified SMI algorithm is designed to maximize MSINR it will "try" to form a pattern null in the interference signal direction. As a result, the gain of the pattern in the interference direction and therefore the interference power will be extremely sensitive to inaccuracy in the covariance estimate. In fact, as $\mathrm{F}$ is increased the null should steepen and the interference power bias and variance should increase. On the other hand, the slope of the pattern in the desired signal direction should be small since the pattern maximum occurs near this direction, hence, the small variance in the desired signal power. If the environment changes slowly, as it does in the satellite communication application, then perhaps the present per- 


\section{OUTPUT INTERFERENCE POWER VS SAMPLE SIZE K}

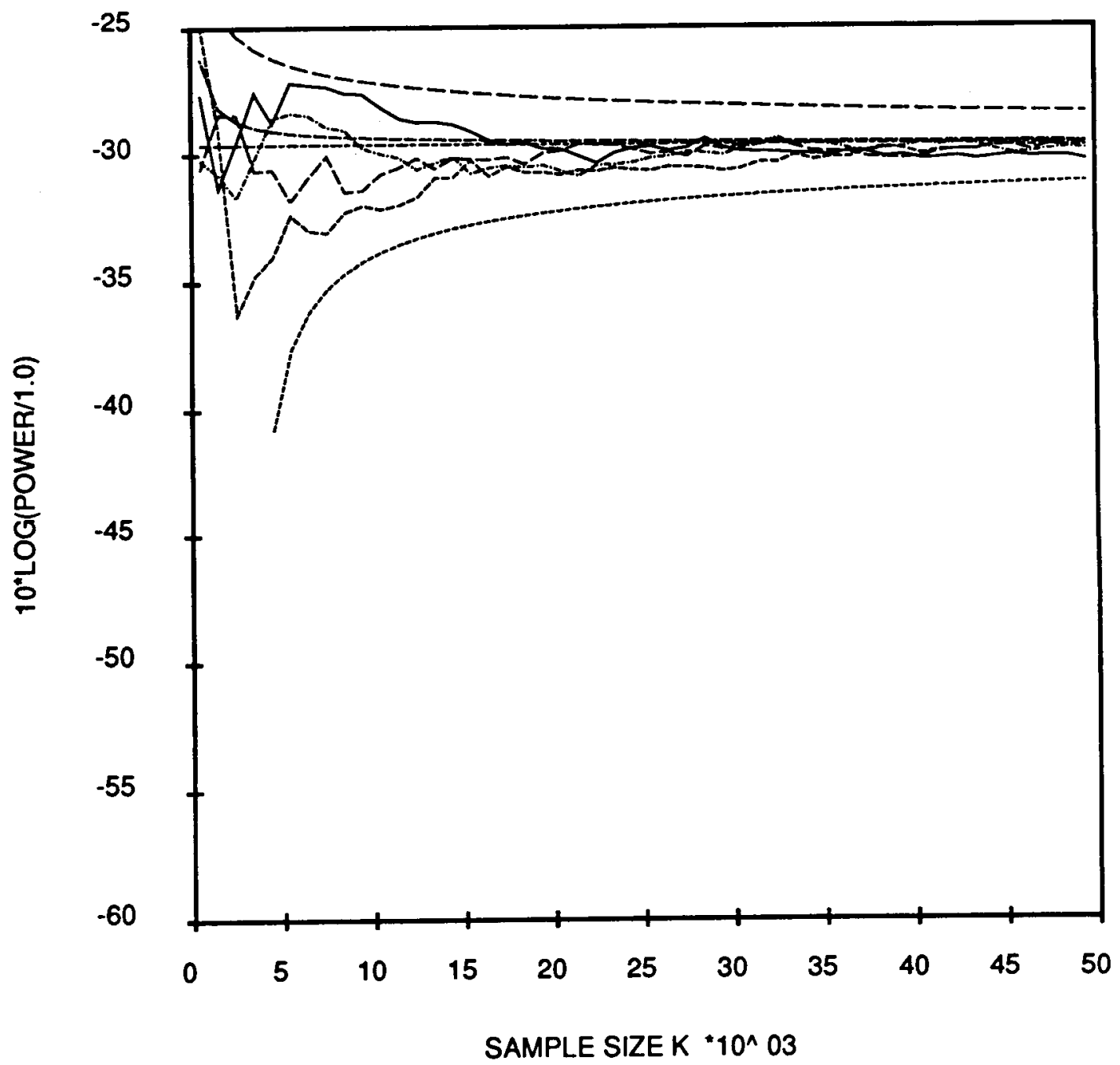

Figure 3.8: Plot of output interference signal power $P_{I}$ versus number of snapshots $K$ for $F=0.0$ shows 4 trials, true covariance curve, bias curve, and $95 \%$ confidence interval. 


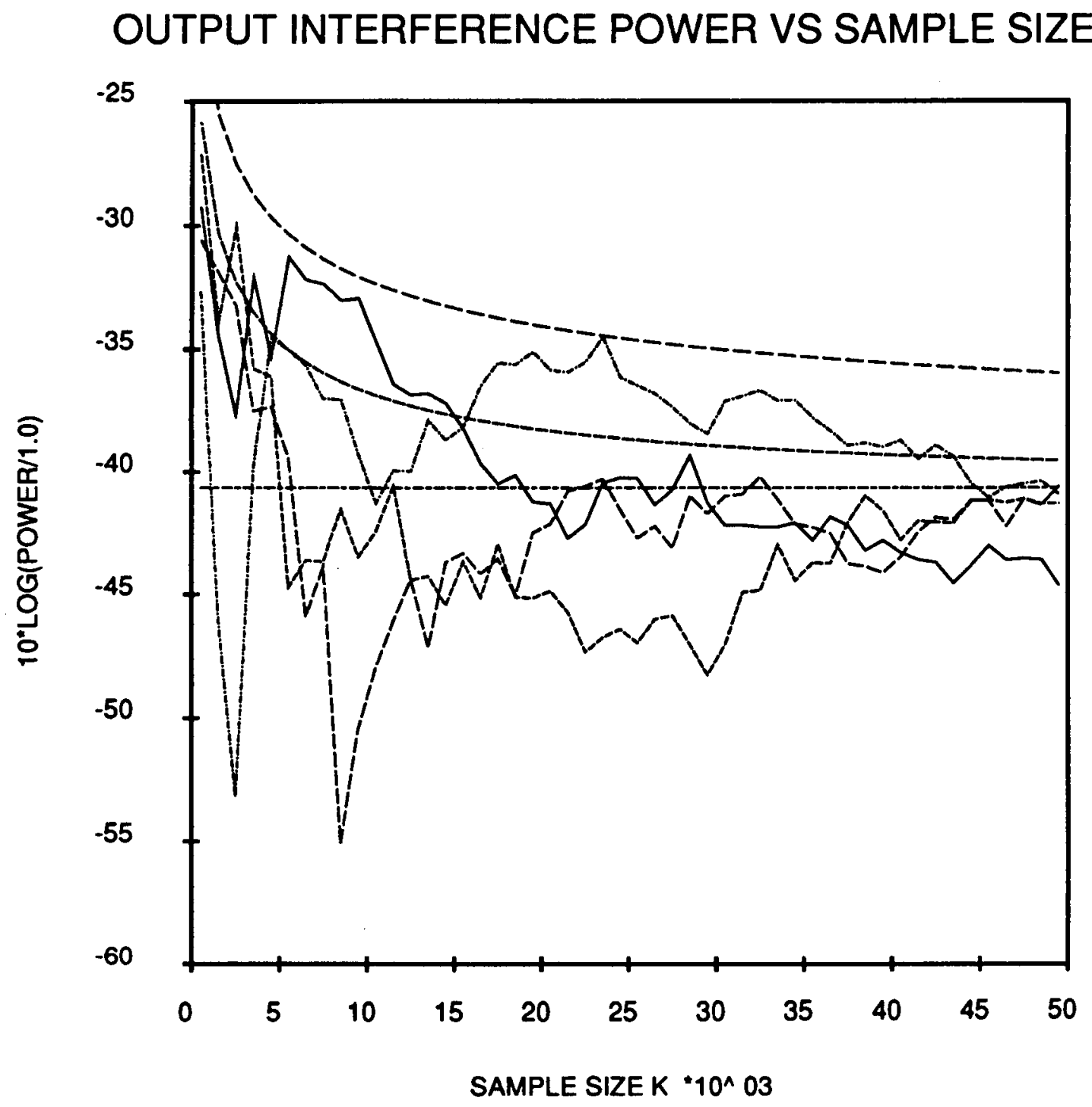

Figure 3.9: Plot of output interference signal power $P_{I}$ versus number of snapshots $K$ for $F=0.8$ shows 4 trials, true covariance curve, bias curve, and $95 \%$ confidence interval. 


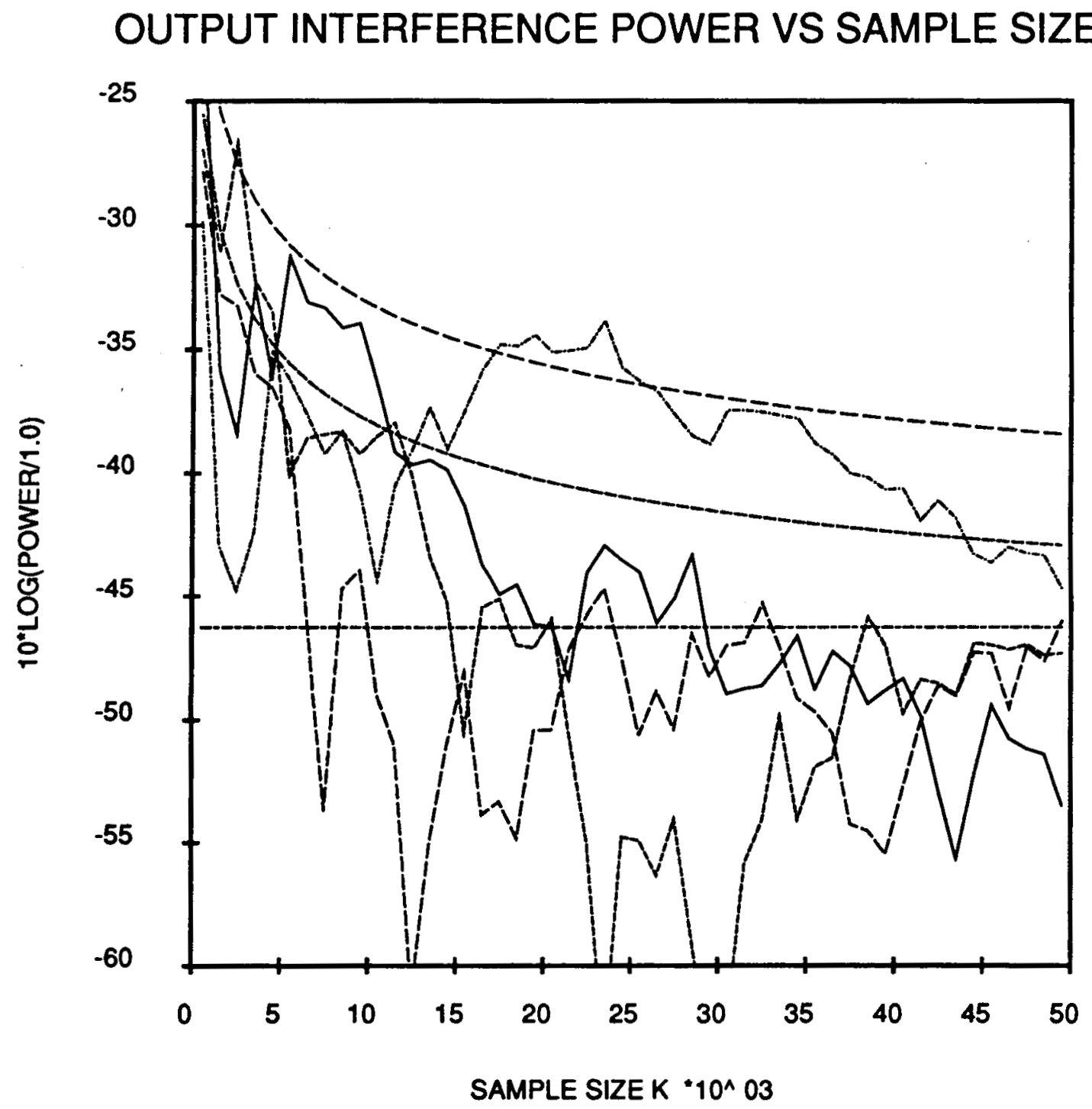

Figure 3.10: Plot of output interference signal power $P_{I}$ versus number of snapshots $K$ for $F=0.9$ shows 4 trials, true covariance curve, bias curve, and $95 \%$ confidence interval. 
formance is satisfactory. However, we shall proceed under the assumption that it is desirable to require fewer snapshots to achieve a certain level of performance.

\subsubsection{Omitting Noise Eigenvectors from the Weight Expression}

Recall from Section 2.3 that the true SMI weights can be written as a linear combination of the eigenvectors of the true covariance $\Phi 2.31$ and that ideally the weights depend only on the true principal eigenvectors. We can take advantage of this a priori knowledge by truncating the sum in 2.31 so as to include just the principal eigenvectors (i.e. change the upper limit from $\mathrm{N}$ to $\mathrm{M}+1$ ). The hope is that by excluding the noise eigenvector estimates, the pattern null in the interference direction may be more stable and hence better interference power performance may result. Of course, one may also argue that the estimated noise subspace $\hat{\mathcal{N}}_{K}$ corresponding to the covariance estimate $\hat{\Phi}_{K}$ will actually have non-zero projection onto the true signal subspace $\mathcal{S}$ and so excluding the noise eigenvector estimates may degrade interference power performance.

The results of the truncation are interesting. Figures 3.14 and 3.15 show the weights on the main and first auxiliary elements, respectively, with all eigenvectors included and $F=0.8$. These are the weights that led to Figures 3.3, 3.6, 3.9, and 3.12. Figures 3.16 and 3.17 are the same weights except that only the principal eigenvectors have been used. Comparing the figures we see that without the noise eigenvectors the array weights have indeed converged much more quickly. However, what really matters 


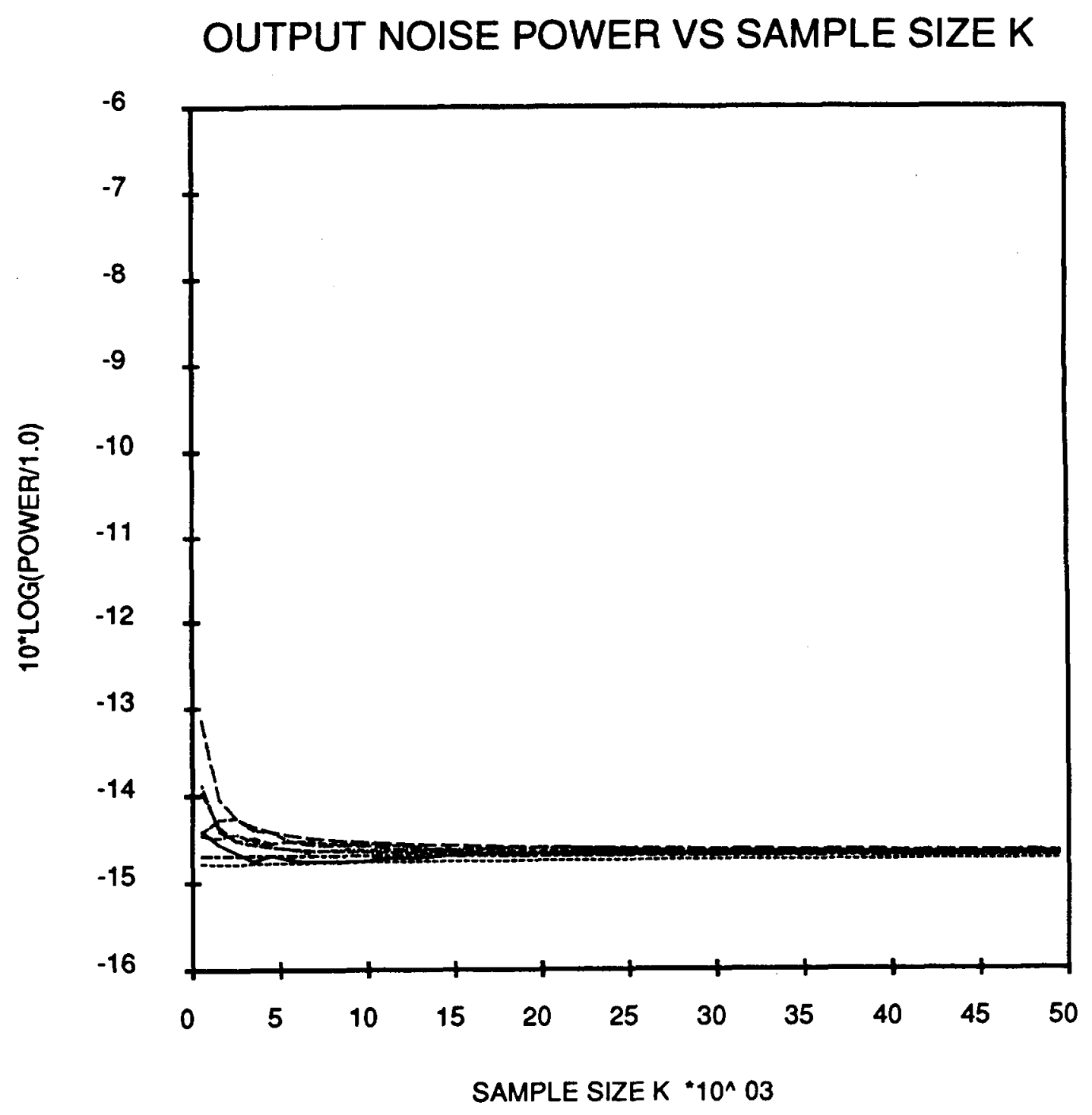

Figure 3.11: Plot of output noise power $P_{\eta}$ versus number of snapshots $K$ for $F=0.0$ shows 4 trials, true covariance curve, bias curve, and $95 \%$ confidence interval. 


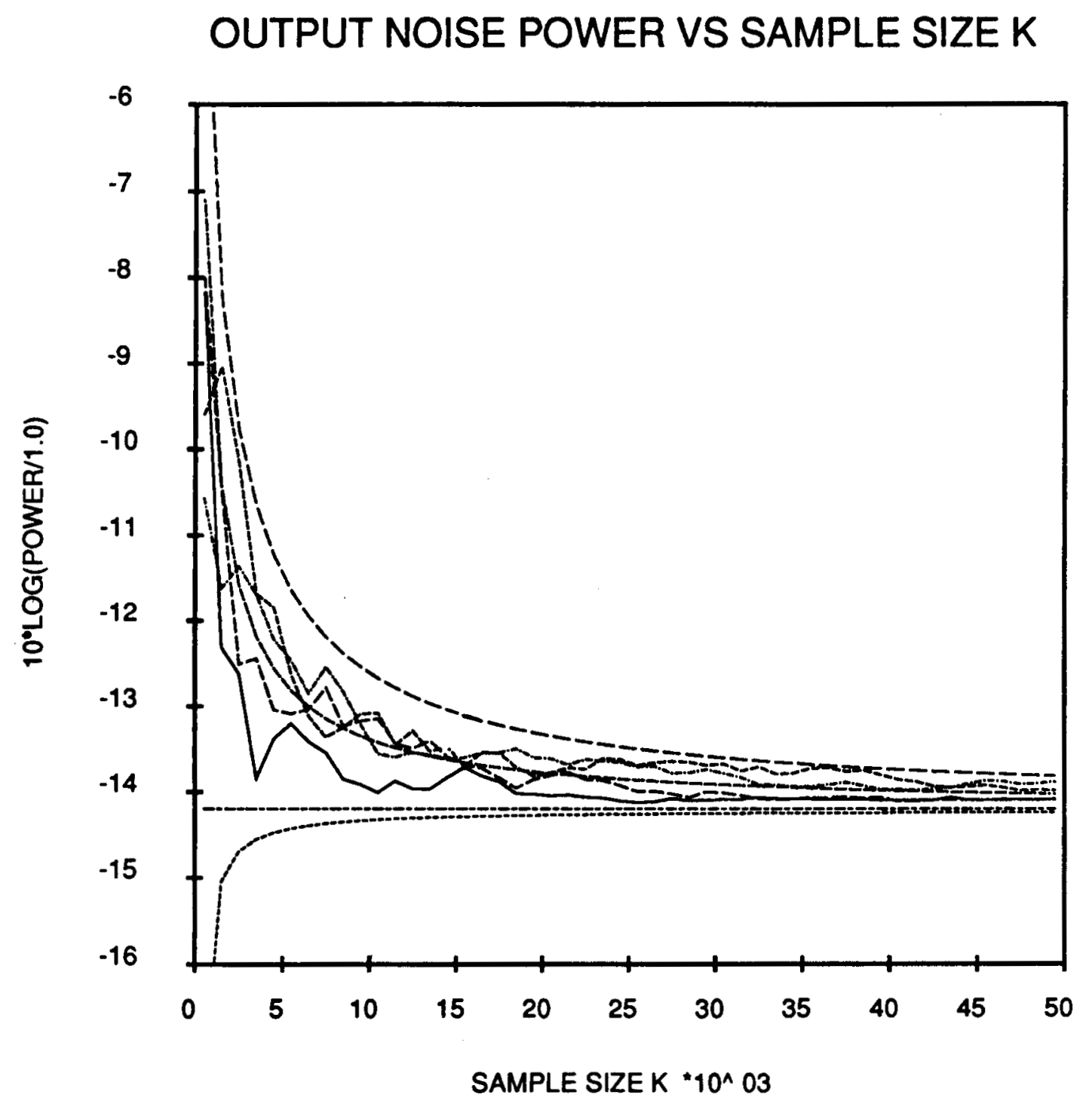

Figure 3.12: Plot of output noise power $P_{\eta}$ versus number of snapshots $K$ for $F=0.8$ shows 4 trials, true covariance curve, bias curve, and $95 \%$ confidence interval. 


\section{OUTPUT NOISE POWER VS SAMPLE SIZE K}

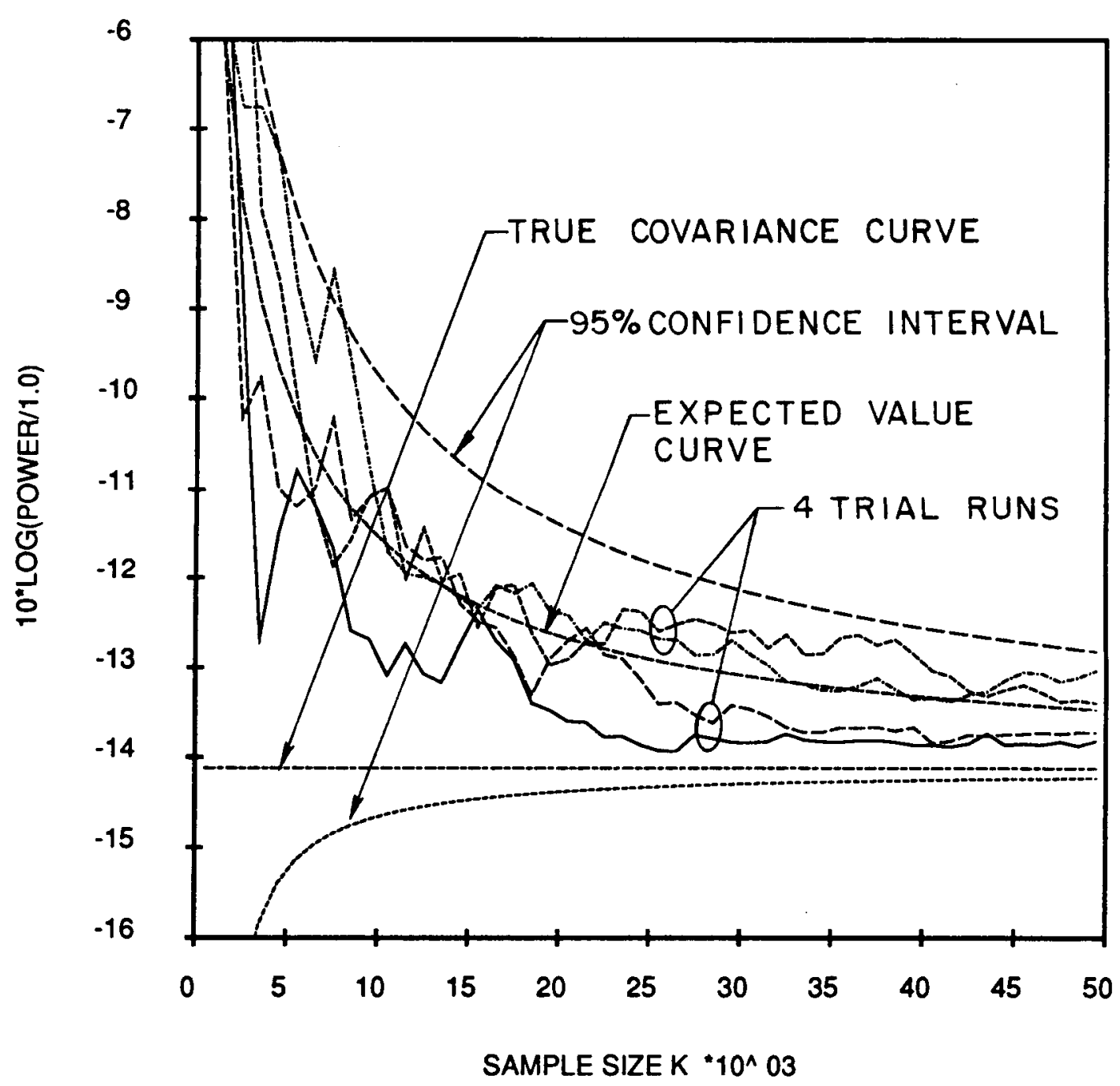

Figure 3.13: Plot of output noise power $P_{\eta}$ versus number of snapshots $K$ for $F=0.9$ shows 4 trials, true covariance curve, bias curve, and $95 \%$ confidence interval. 


\section{MAIN ANTENNA WEIGHT VS SAMPLE SIZE K}

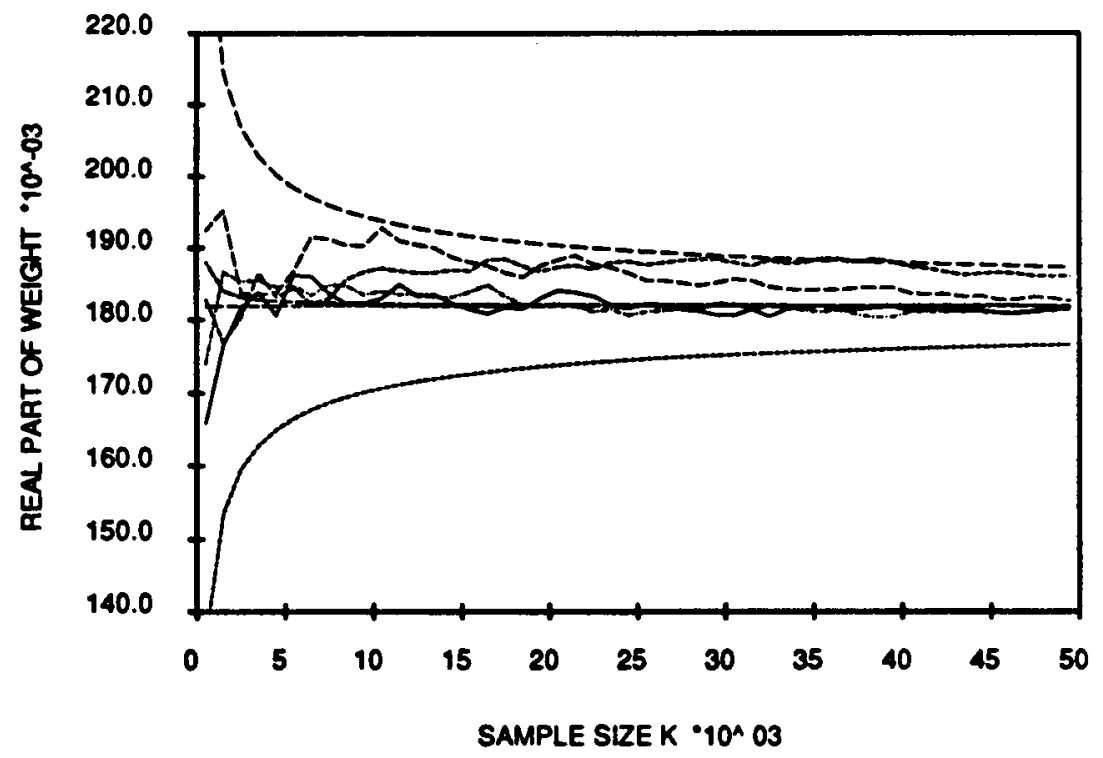

\section{MAIN ANTENNA WEIGHT VS SAMPLE SIZE K}

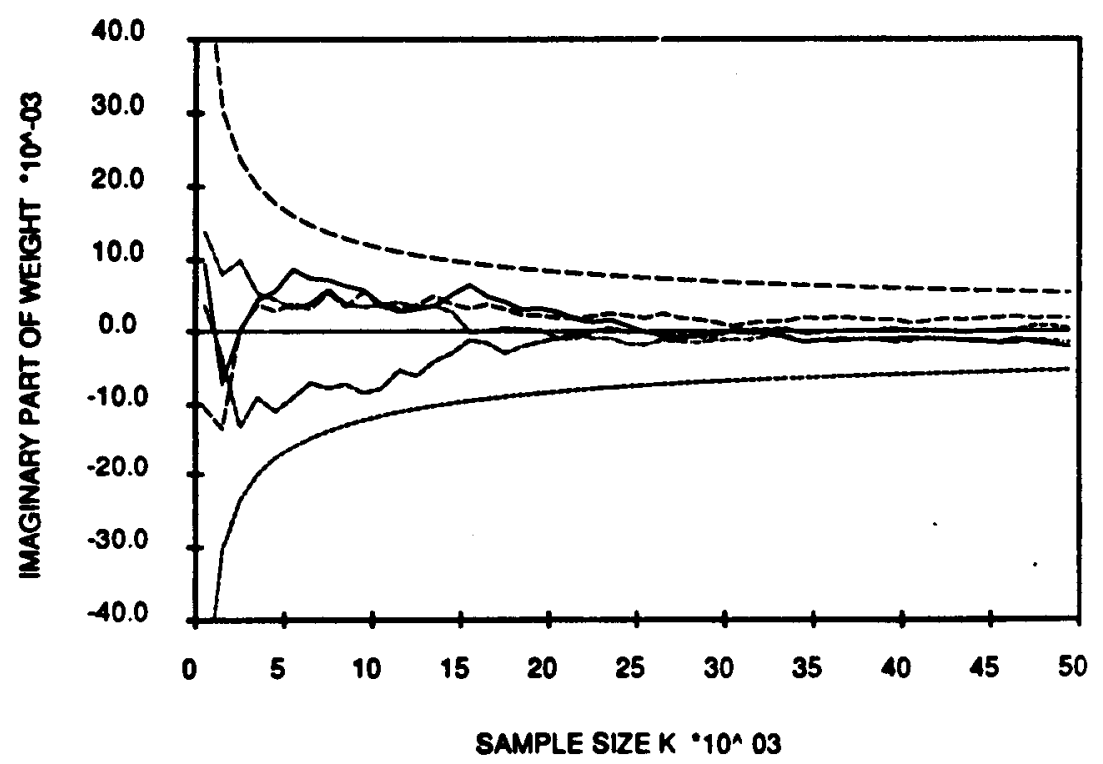

Figure 3.14: Real and imaginary parts of main element weight $W_{1}$ versus number of snapshots $K$ for $F=0.8$ shows 4 trials, true covariance curve, bias curve, and $95 \%$ confidence interval. All eigenvectors are used in the weight expression. 


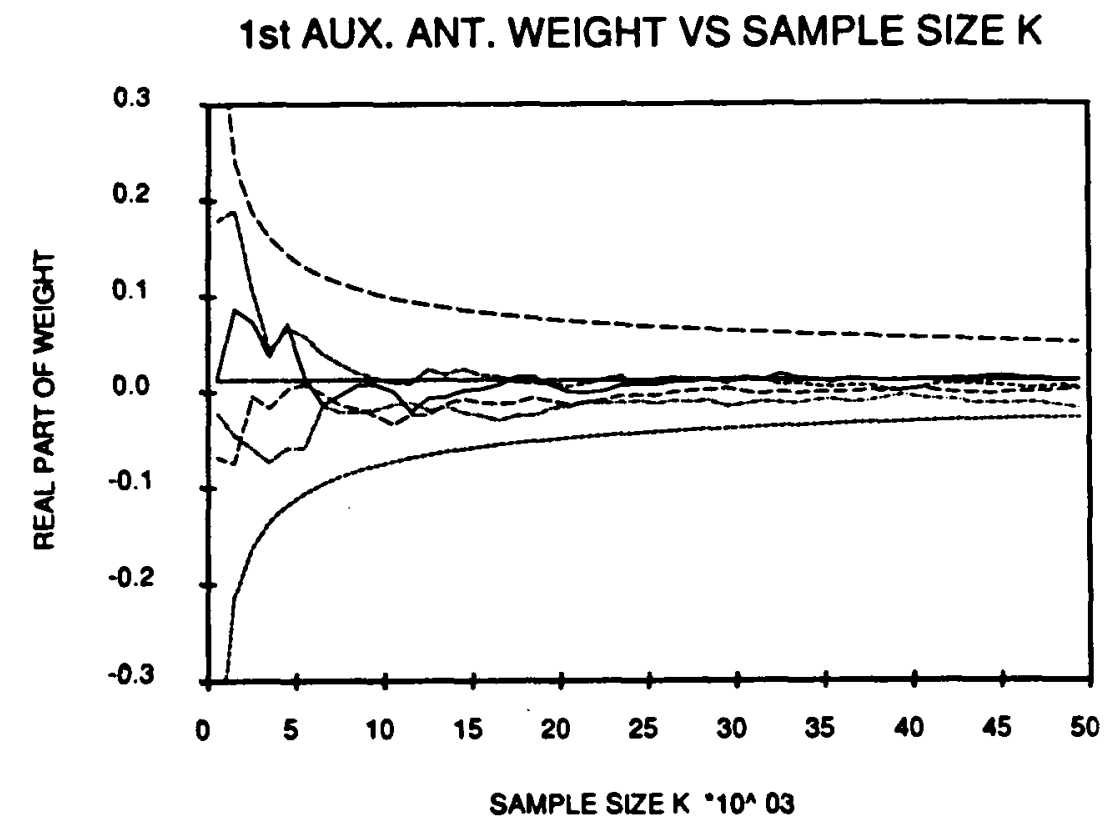

1st AUX. ANT. WEIGHT VS SAMPLE SIZE K

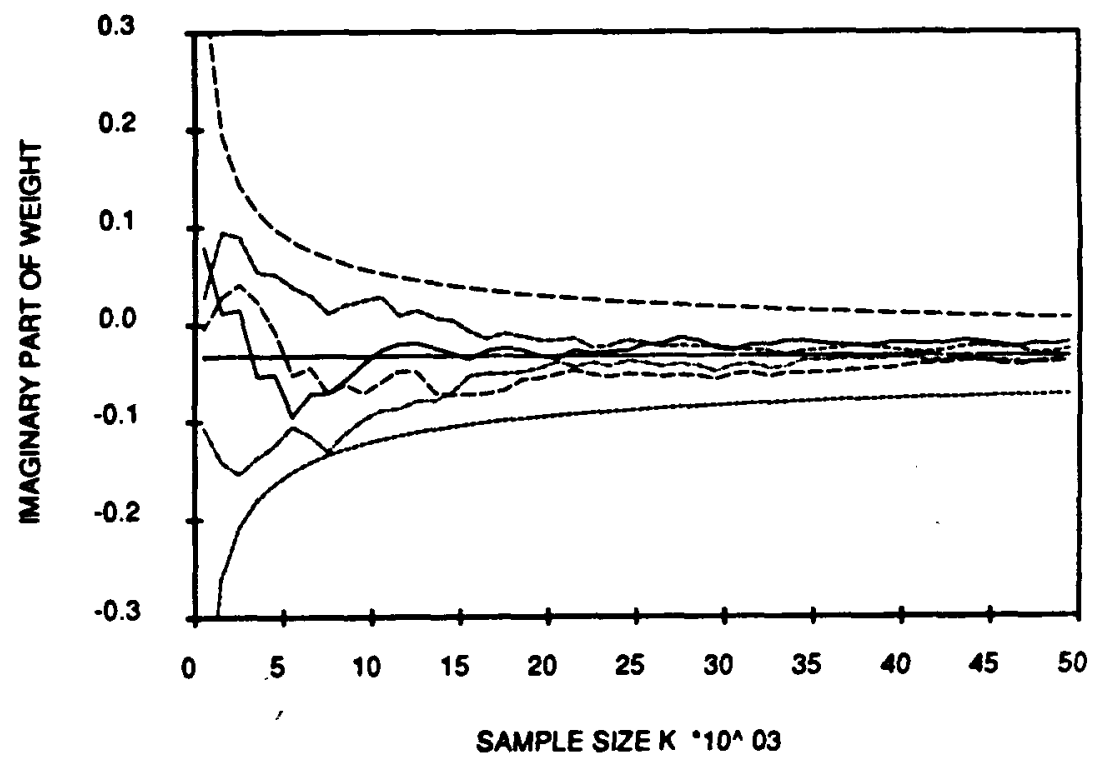

Figure 3.15: Real and imaginary parts of first auxiliary element weight $W_{2}$ versus number of snapshots $K$ for $F=0.8$ shows 4 trials, true covariance curve, bias curve, and $95 \%$ confidence interval. All eigenvectors are used in the weight expression. 

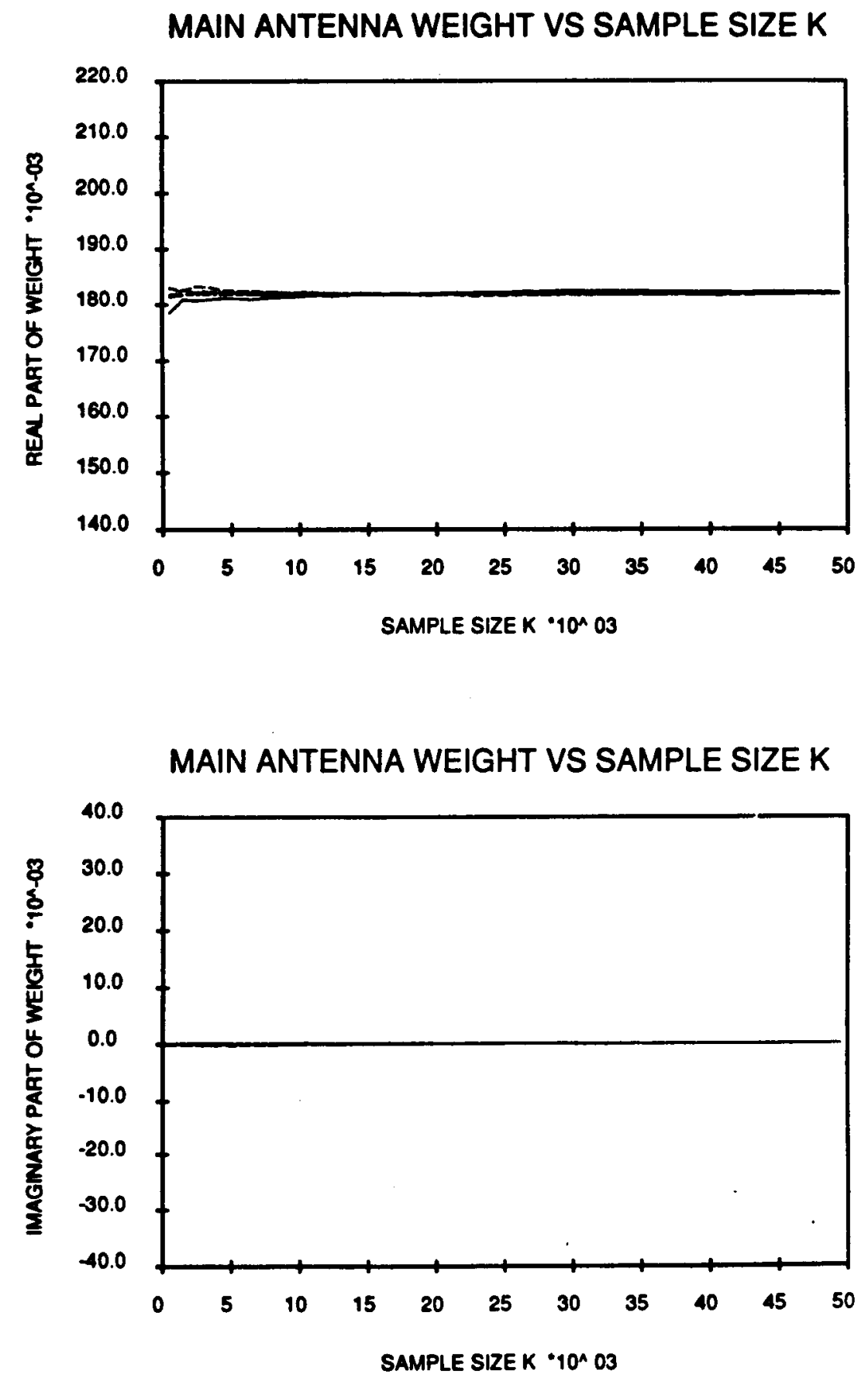

Figure 3.16: Real and imaginary parts of main element weight $W_{1}$ versus number of snapshots $K$ for $F=0.8$ shows 4 trials and true covariance curve. Only principal eigenvectors are used in the weight expression. 

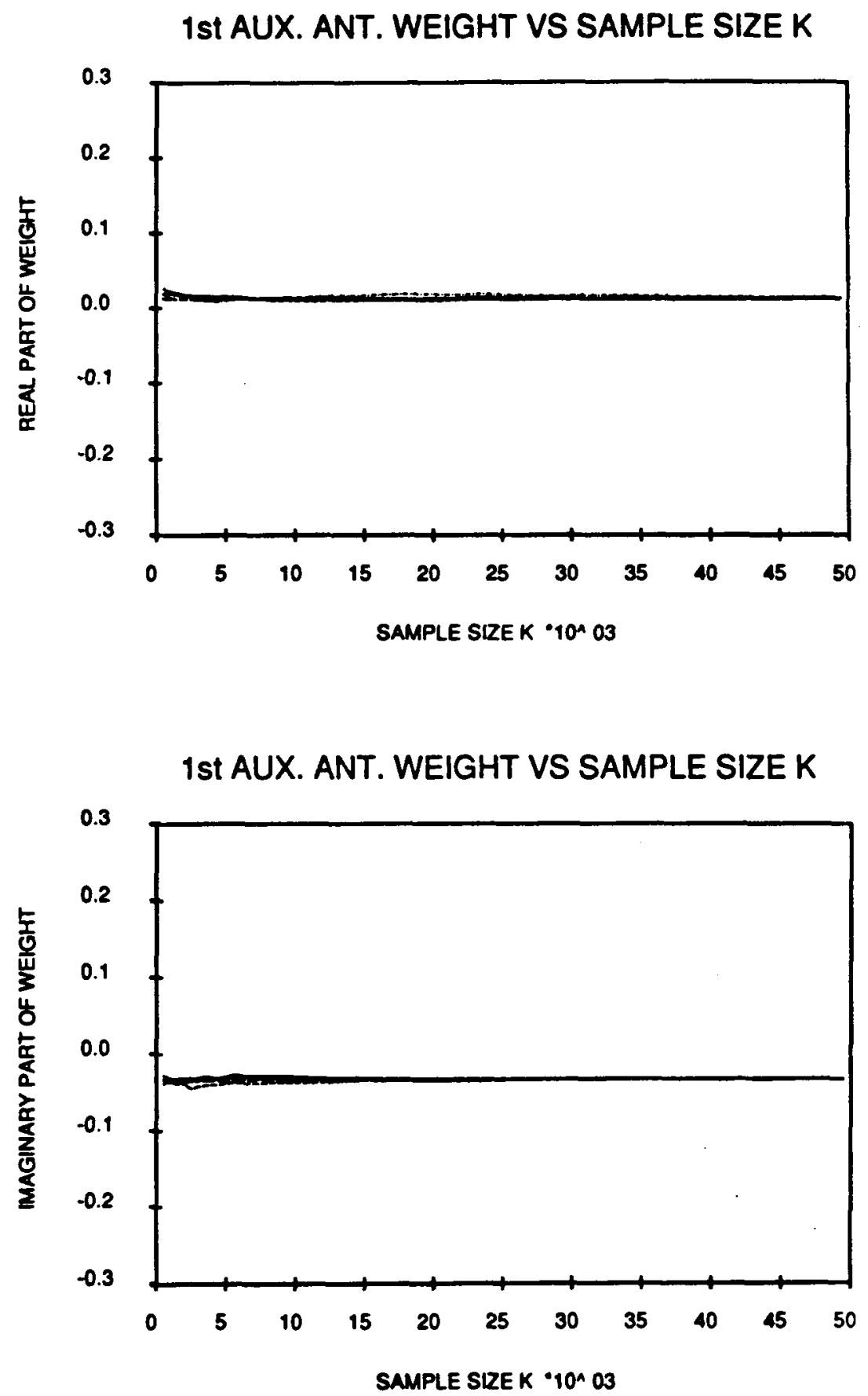

Figure 3.17: Real and imaginary parts of first auxiliary element weight $W_{2}$ versus number of snapshots $K$ for $F=0.8$ shows 4 trials and true covariance curve. Only principal eigenvectors are used in the weight expression. 
is the performance of the array with respect to the output powers. Figures 3.18-3.20 show the output powers of the array corresponding to the "calm" weights based on only the principal eigenvectors. Compare these with Figures $3.6,3.9$, and 3.12 , respectively. Somewhat surprisingly, what seems to be a significant improvement in the weight performance has led to very little change in the desired and interference signal powers, although it has made the noise power performance ideal.

To understand these observations, consider the desired and interference portions of the array output signal where the estimated weights of 3.20 have been used. From 2.11, 2.4, 2.5, 2.16, and 2.31 we may write

$$
\begin{aligned}
\hat{s}_{D}+\sum_{m=1}^{M} \hat{s}_{I m}= & \hat{W}^{H}\left[X_{D}+\sum_{m=1}^{M} X_{I m}\right] \\
= & {\left[\mu^{*} \sum_{n=1}^{M+1}\left(\frac{\hat{e}_{n}^{H} S_{D}}{\hat{\lambda}_{n}-F \sigma^{2}}\right)^{*} \hat{e}_{n}^{H}\right]\left[c_{D} S_{D}+\sum_{m=1}^{M} c_{m} S_{I m}\right] } \\
+ & {\left[\mu^{*} \sum_{n=M+2}^{N}\left(\frac{\hat{e}_{n}^{H} S_{D}}{\hat{\lambda}_{n}-F \sigma^{2}}\right)^{*} \hat{e}_{n}^{H}\right] } \\
& {\left[c_{D} S_{D}+\sum_{m=1}^{M} c_{m} S_{I m}\right] }
\end{aligned}
$$

where the c's are some unity-magnitude scalars and the true covariance eigen-decomposition has been replaced with its $\mathrm{K}$-snapshot-based estimate. In the final expression, the third bracketed term contains the weight components (conjugate transposed) that are left out in the noise eigenvector truncation and thus the second line of the last expression gives the components of the output desired and interference signals that are excluded by the truncation. If the true covariance were used, the third bracketed term would be zero since $e_{n}^{H} S_{D}=0$ for $M+2 \leq n \leq N$. However, since estimated 
eigenvectors are used, the third bracketed term is not zero and in fact is significant because of the small denominator $\hat{\lambda}_{n}-F \sigma^{2}$. Hence, upon omitting the noise eigenvectors the weights calmed down notably. Though the third bracketed term of 3.21 causes noticeable "jumpiness" in the weights, it multiplies an approximately orthogonal vector (the fourth bracketed term of 3.21) so that the second line of 3.21 is small. This observation explains why excluding the noise eigenvectors did not significantly affect the output desired and interference signal powers. From the array pattern perspective, excluding the noise eigenvectors from the array weight expression does not greatly affect the array pattern in the signal and interference directions. In almost all other directions, however, the pattern does "calm down" resulting in the greatly improved noise power performance which we observed. In conclusion, although we cannot hope to significantly improve interference power performance by excluding noise eigenvectors from the weight estimate, we can significantly improve array performance with respect to output noise power.

\subsubsection{Characterization of Sample Covariance Errors}

Can the type of error occurring in the sample covariance matrix $\hat{\Phi}_{K}$ be characterized? To address this question we express the sample covariance as 


\section{OUTPUT SIGNAL POWER VS SAMPLE SIZE K}

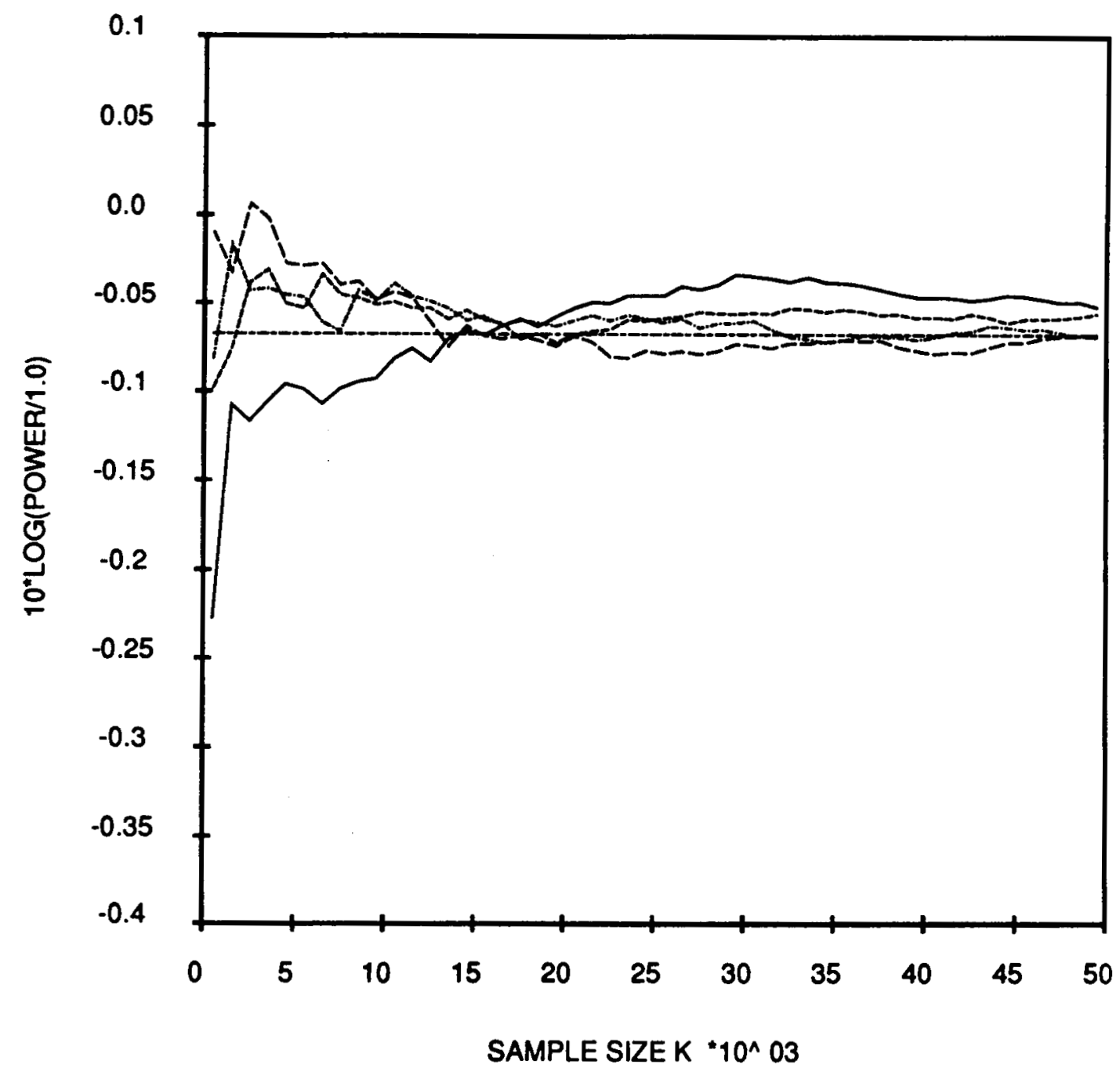

Figure 3.18: Plot of output desired signal power $P_{D}$ versus number of snapshots $K$ for $F=0.8$ shows 4 trials and the true covariance curve. Weights were found using only the signal eigenvectors. 


\section{OUTPUT INTERFERENCE POWER VS SAMPLE SIZE K}

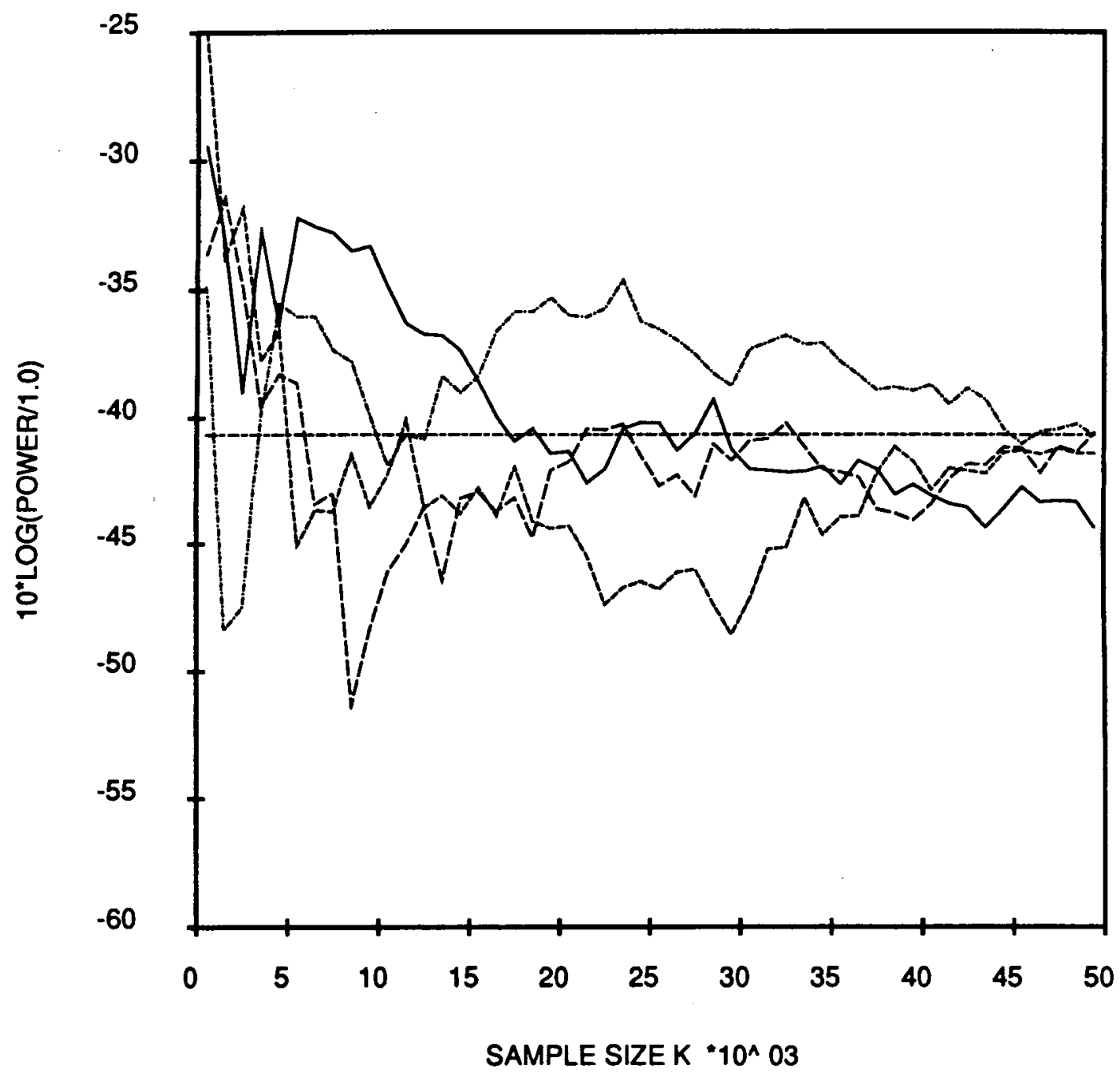

Figure 3.19: Plot of output interference signal power $P_{I}$ versus number of snapshots $K$ for $F=0.8$ shows 4 trials and the true covariance curve. Weights were found using only the signal eigenvectors. 
OUTPUT NOISE POWER VS SAMPLE SIZE K

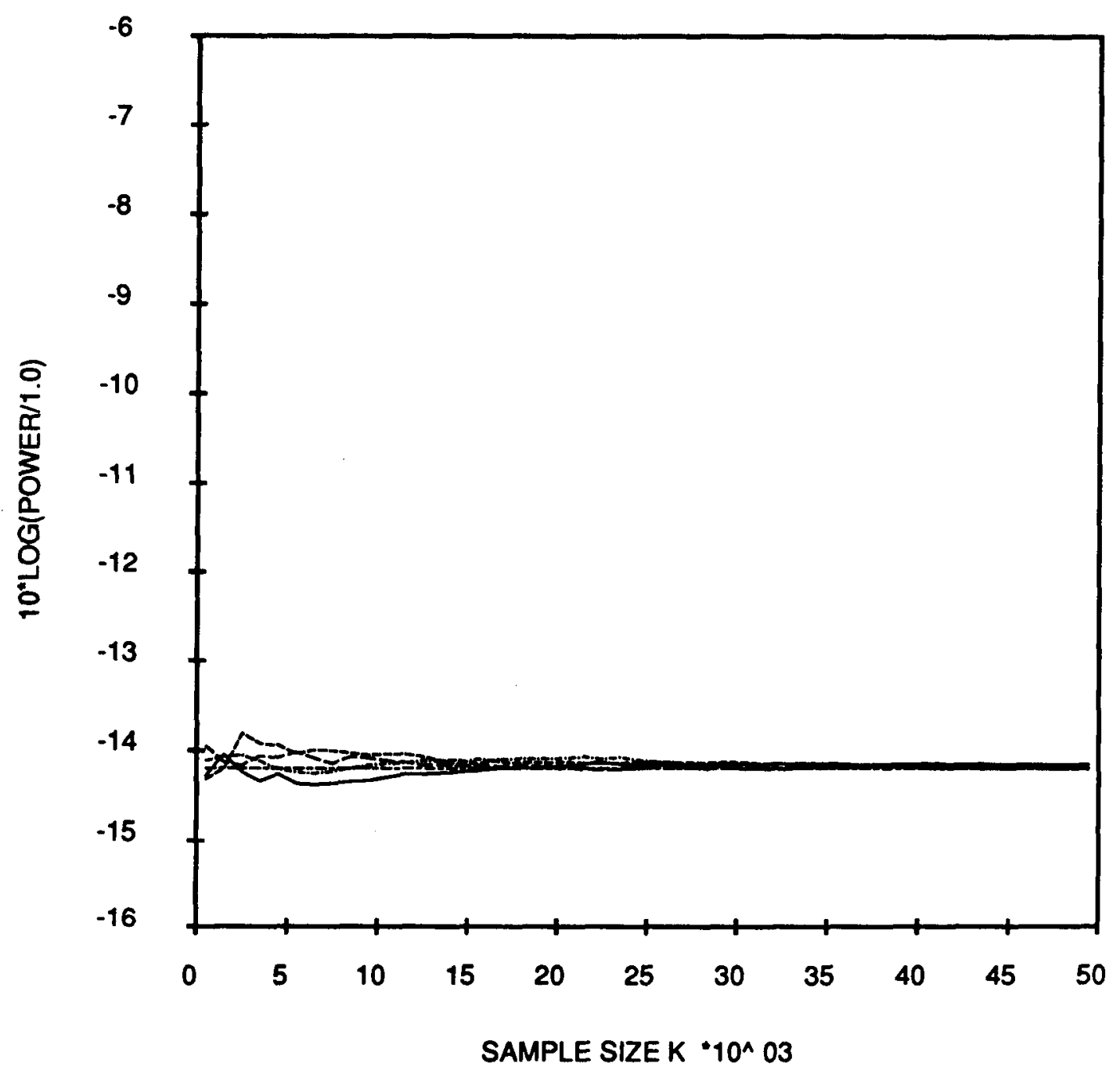

Figure 3.20: Plot of output noise power $P_{\eta}$ versus number of snapshots $K$ for $F=0.8$ shows 4 trials and the true covariance curve. Weights were found using only the signal eigenvectors. 


$$
\begin{aligned}
\hat{\Phi}_{K}= & \frac{1}{K} \sum_{k=1}^{K}\left(X_{D k}+X_{I 1 k}+X_{\eta k}\right)\left(X_{D k}+X_{I 1 k}+X_{\eta k}\right)^{H} \\
= & \Phi_{D}+\Phi_{I 1}+\frac{1}{K} \sum_{k=1}^{K} X_{\eta k} X_{\eta k}^{H} \\
& +\frac{1}{K} \sum_{k=1}^{K}\left[X_{D k} X_{I 1 k}^{H}+X_{I 1 k} X_{D k}^{H}\right] \\
& +\frac{1}{K} \sum_{k=1}^{K}\left[X_{D k} X_{\eta k}^{H}+X_{\eta k} X_{D k}^{H}\right] \\
& +\frac{1}{K} \sum_{k=1}^{K}\left[X_{I 1 k} X_{\eta k}^{H}+X_{\eta k} X_{I 1 k}^{H}\right]
\end{aligned}
$$

using $3.17,3.18$, and 3.19 in 3.1 with one interference signal $(M=1)$. The first line of the last expression approaches $\Phi$ with probability 1 as $K \rightarrow \infty$ whereas the rest of the terms approach 0 . We shall say that the second line consists of the desired-interference crossterms (DICT), the third line consists of desired-noise crossterms (DNCT), and the last line consists of interference-noise crossterms (INCT). Since the formation of $\hat{\boldsymbol{\Phi}}_{K}$ is under the programmer's control it is possible to observe the effect of a particular type of crossterm (for example DICT, DNCT, or INCT) by omitting the other crossterms of 3.22. Figures 3.21-3.24 show the power ratios and powers if the sample covariance matrix is formed as $\hat{\Phi}_{K}=\Phi_{D}+$ $\Phi_{I 1}+(1 / K) \sum_{k=1}^{K} X_{\eta k} X_{\eta k}^{H}$. Similarly, Figures 3.25-3.28, 3.29-3.32, and 3.33, show the results for forming $\hat{\Phi}_{K}=\Phi+\operatorname{DICT}, \hat{\Phi}_{K}=\Phi+$ DNCT, and $\hat{\Phi}_{K}=\Phi+$ INCT, respectively.

From Figures 3.21-3.24 we see that the error in the estimate of $\Phi_{\eta}$ causes only a moderate degradation in performance when compared to the "all- 


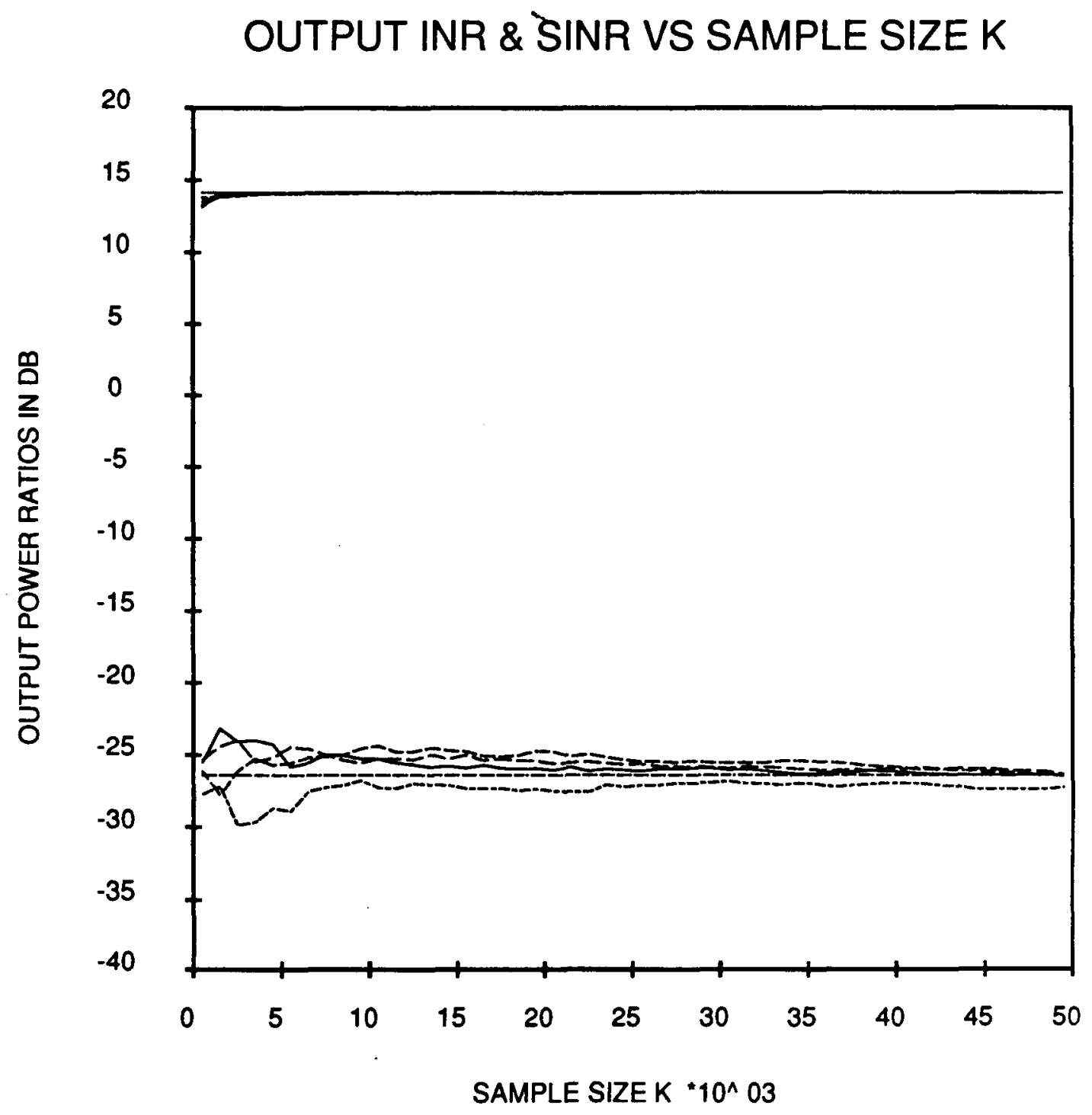

Figure 3.21: Plot of output INR and SINR versus number of snapshots $K^{-}$ for $F=0.8$ and $\hat{\Phi}_{K}=\Phi_{D}+\Phi_{I 1}+(1 / K) \sum_{k=1}^{K} X_{\eta k} X_{\eta k}^{H}$. Shows 4 simulations and the true covariance curve. 


\section{OUTPUT SIGNAL POWER VS SAMPLE SIZE K}

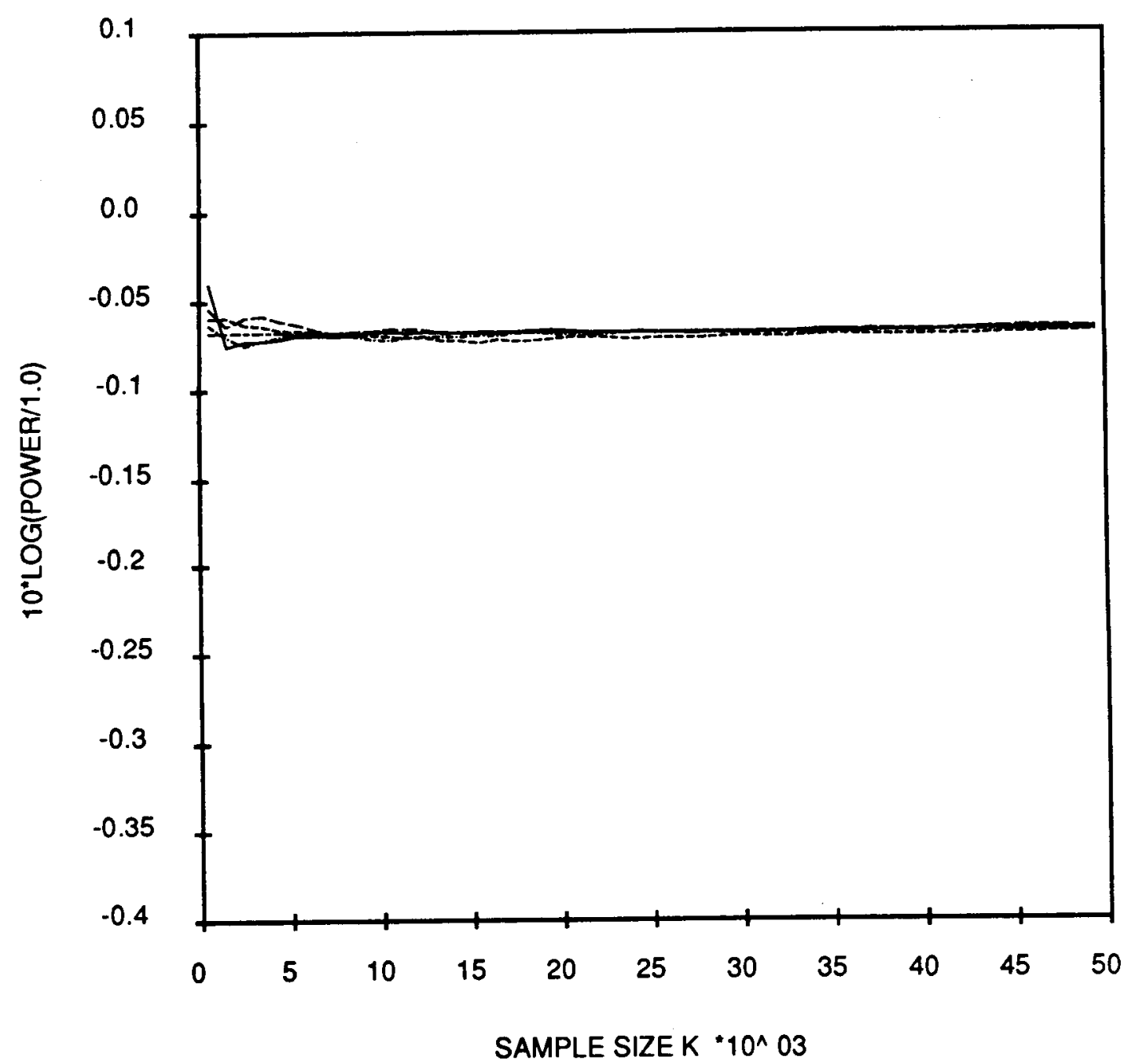

Figure 3.22: Plot of output desired signal power $P_{D}$ versus number of snapshots $K$ for $F=0.8$ and $\hat{\Phi}_{K}=\Phi_{D}+\Phi_{I 1}+(1 / K) \sum_{k=1}^{K} X_{\eta k} X_{\eta k}^{H}$. Shows 4 simulations and the true covariance curve. 


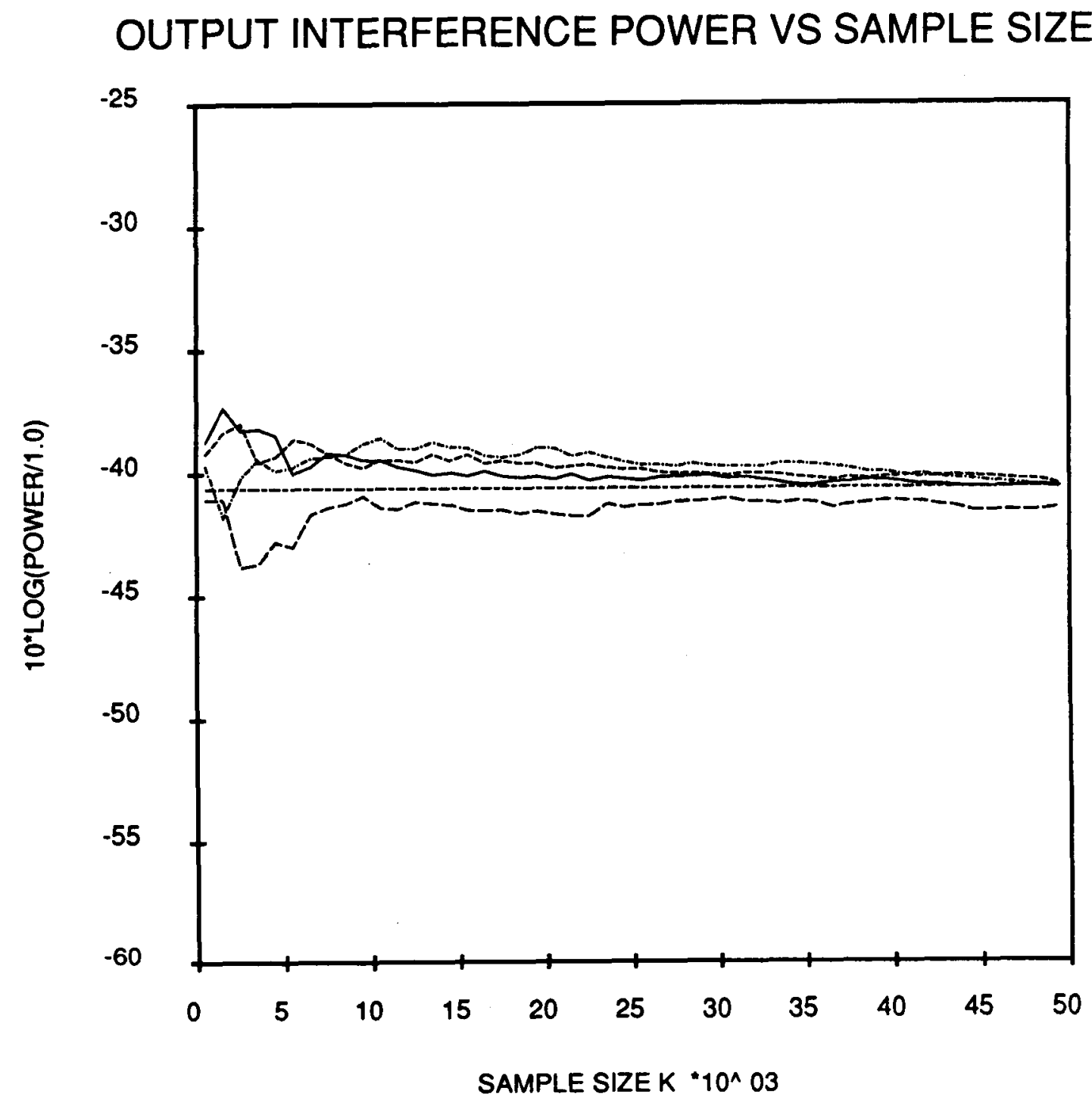

Figure 3.23: Plot of output interference signal power $P_{I}$ versus number of snapshots $K$ for $F=0.8$ and $\hat{\Phi}_{K}=\Phi_{D}+\Phi_{I 1}+(1 / K) \sum_{k=1}^{K} X_{\eta k} X_{\eta k}^{H}$. Shows 4 simulations and the true covariance curve. 


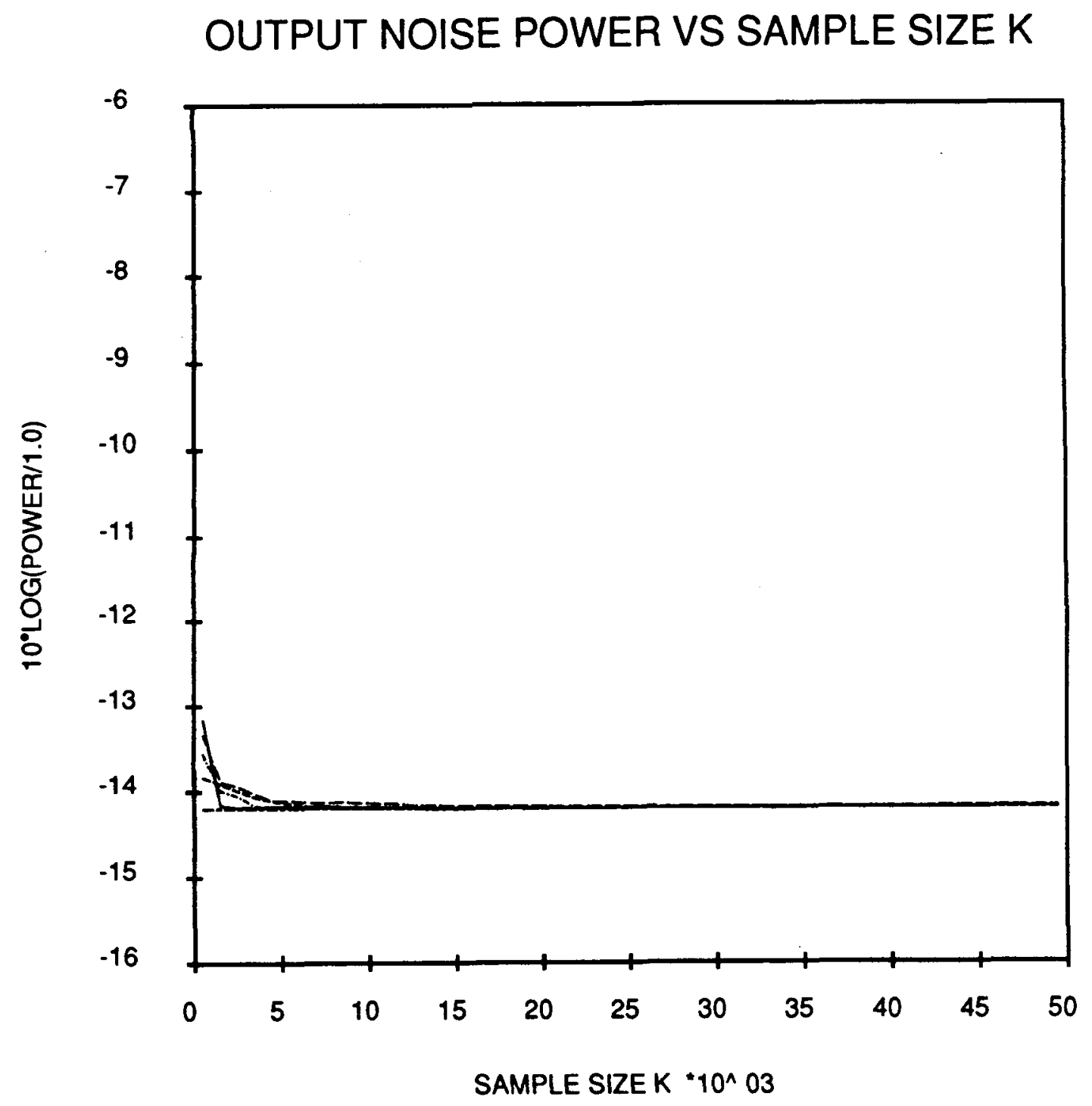

Figure 3.24: Plot of output noise power $P_{\eta}$ versus number of snapshots $K$ for $F=0.8$ and $\hat{\Phi}_{K}=\Phi_{D}+\Phi_{I 1}+(1 / K) \sum_{k=1}^{K} X_{\eta k} X_{\eta k}^{H}$. Shows 4 simulations and the true covariance curve. 
crossterms" performance shown in Figures 3.3, 3.6, 3.9, and 3.12. The performance of each power is degraded since any crossterms involving noise will alter both noise and signal eigenvectors of $\hat{\Phi}_{K}$. The power ratios for the INCT-only simulations appear in Figure 3.33 where only the sensitive interference powers are non-ideal.

The above errors are relatively small because $\left\|X_{\eta k}\right\|,\left\|X_{I 1 k}\right\| \ll\left\|X_{D k}\right\|$ since we are considering weak interference and 14.6dB SNR in the main element. By the same reasoning, one would argue that the performance degradation due to the DICT and DNCT should be relatively large since they both involve the desired signal and, in addition, should be of the same order since the interference and noise powers are about the same. This is somewhat the case as seen in Figures 3.25-3.32. Again, all powers are affected by the DNCT because noise is involved. Notice that the DICT which were used in Figures 3.25-3.28 seem to exclusively affect the interference power. One (as yet unjustified) explanation for this observation is that the DICT tend to alter the signal eigenvectors more than the noise eigenvectors. Small deviations in the signal eigenvectors can lead to big jumps in interference power since the interference enters the array in the vicinity of a null whereas the desired signal power may remain calm because the pattern maximum occurs near the desired signal arrival angle.

Earlier it was implied that crossterms involving the desired signal tend to be relatively large because $\left\|X_{D k}\right\|$ is relatively large. We can be more specific by noting that it is only the first element of $X_{D k}$ that is large due to the high gain of the main array element required for a strong desired signal. 


\section{OUTPUT INR \& SINR VS SAMPLE SIZE K}

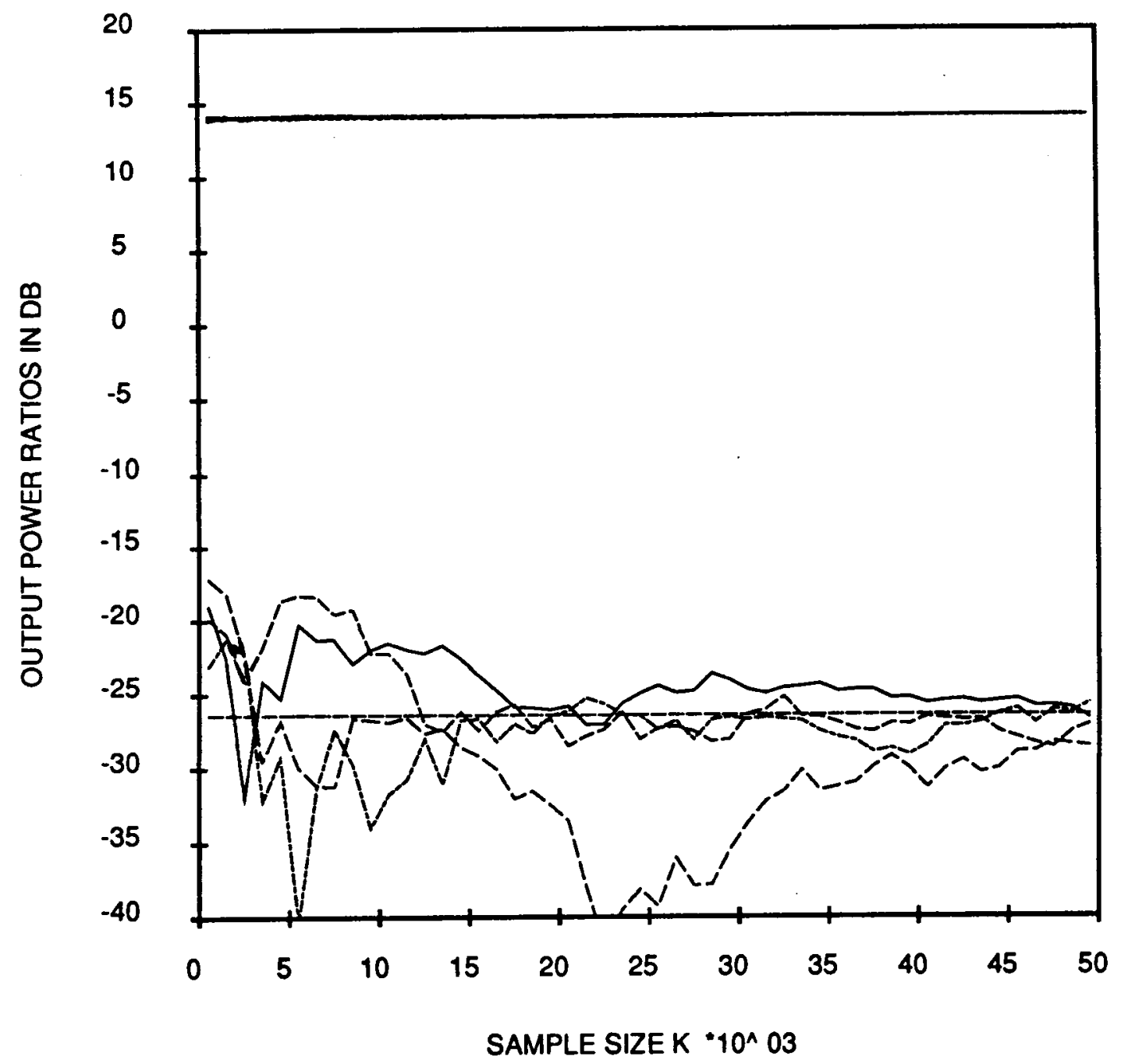

Figure 3.25: Plot of output INR and SINR versus number of snapshots $K$ for $F=0.8$ and $\hat{\Phi}_{K}=\Phi+$ DICT. Shows 4 simulations and the true covariance curve. 


\section{OUTPUT SIGNAL POWER VS SAMPLE SIZE K}

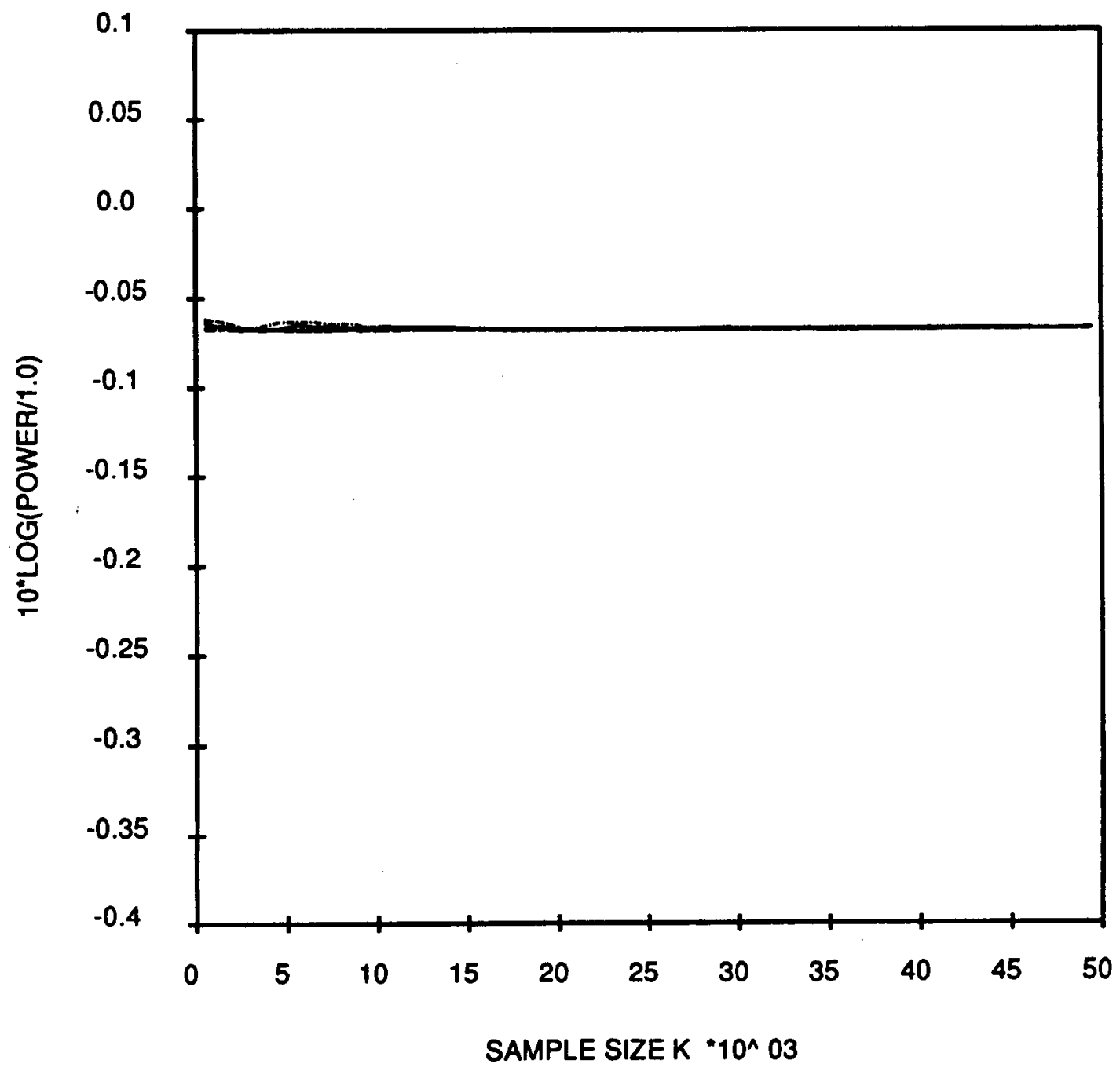

Figure 3.26: Plot of output desired signal power versus number of snapshots $K$ for $F=0.8$ and $\hat{\Phi}_{K}=\Phi+$ DICT. Shows 4 simulations and the true covariance curve. 


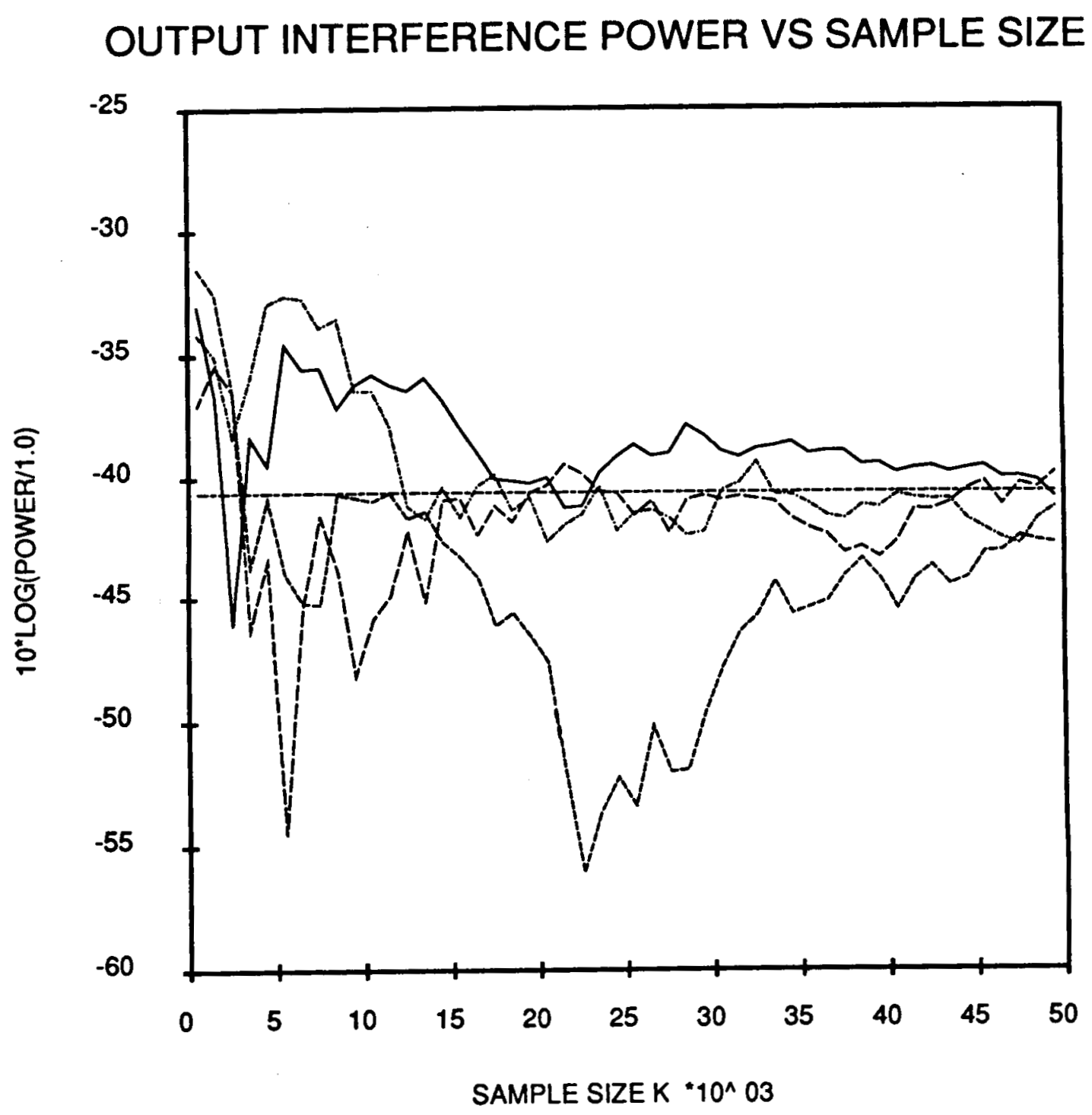

Figure 3.27: Plot of output interference power versus number of snapshots $K$ for $F=0.8$ and $\hat{\Phi}_{K}=\Phi+$ DICT. Shows 4 simulations and the true covariance curve. 
OUTPUT NOISE POWER VS SAMPLE SIZE K

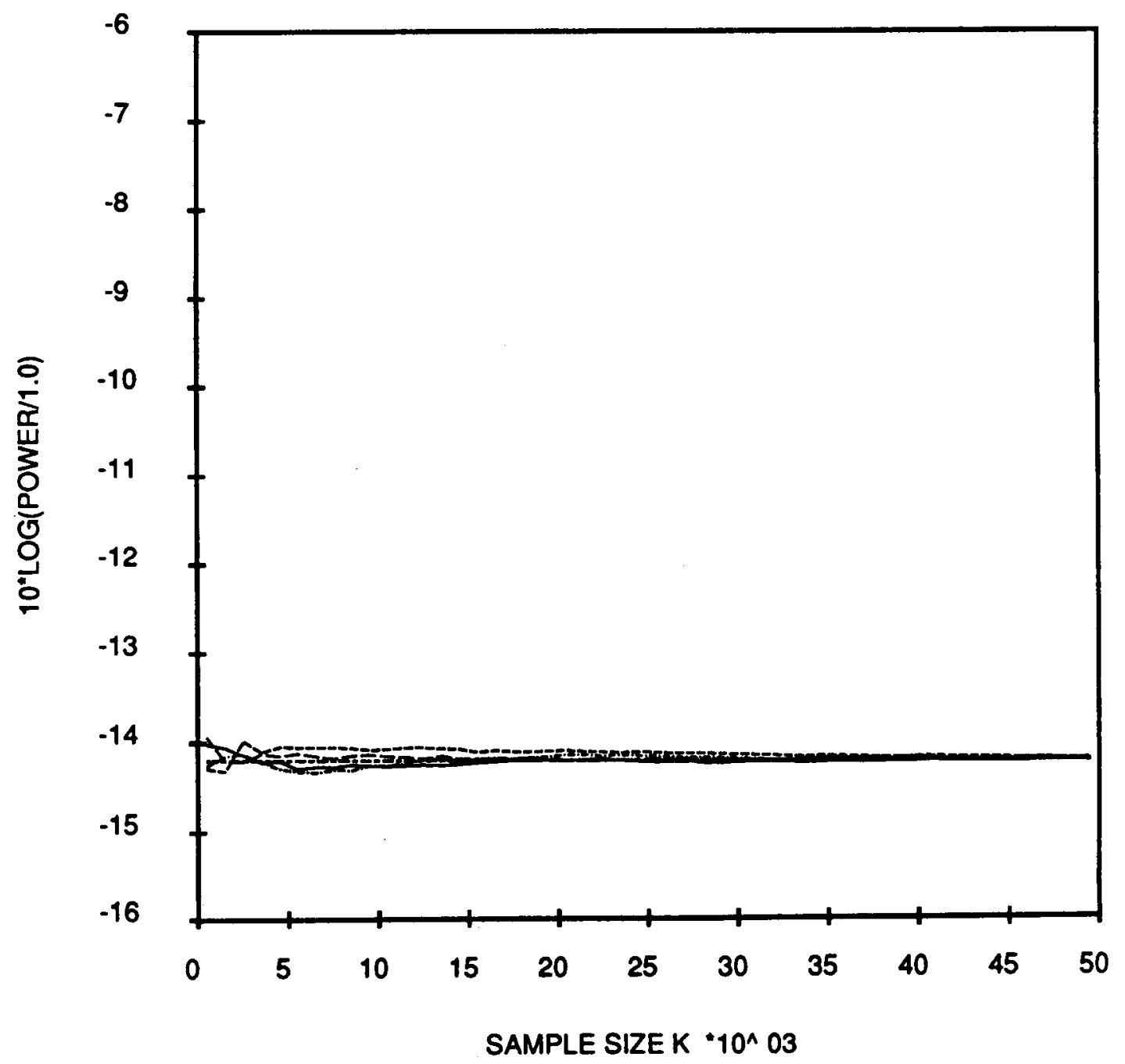

Figure 3.28: Plot of output noise power versus number of snapshots $K$ for $F=0.8$ and $\hat{\Phi}_{K}=\Phi+$ DICT. Shows 4 simulations and the true covariance curve. 


\section{OUTPUT INR \& SINR VS SAMPLE SIZE K}

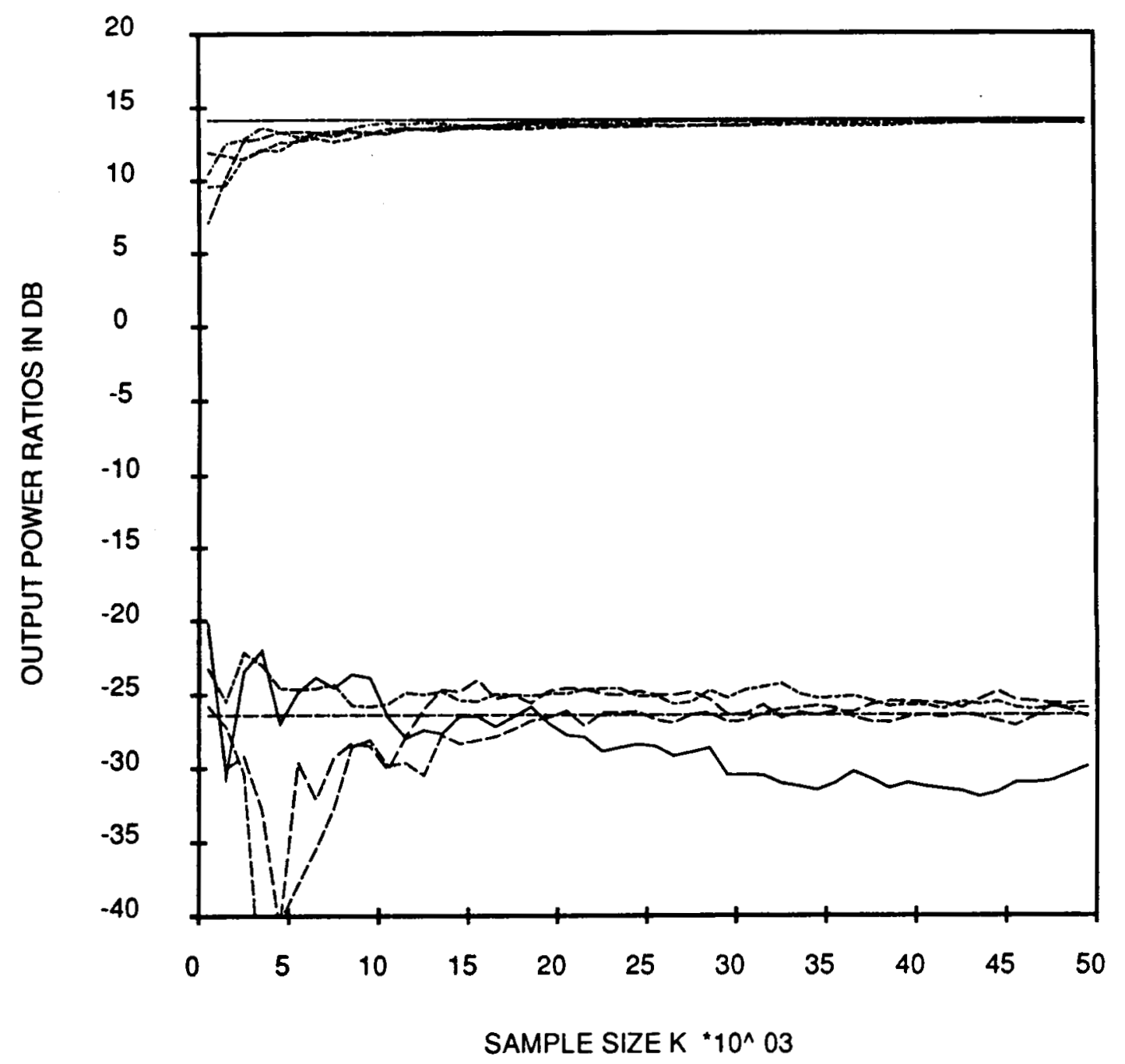

Figure 3.29: Plot of output INR and SINR versus number of snapshots $K$ for $F=0.8$ and $\hat{\Phi}_{K}=\Phi+$ DNCT. Shows 4 simulations and the true covariance curve. 


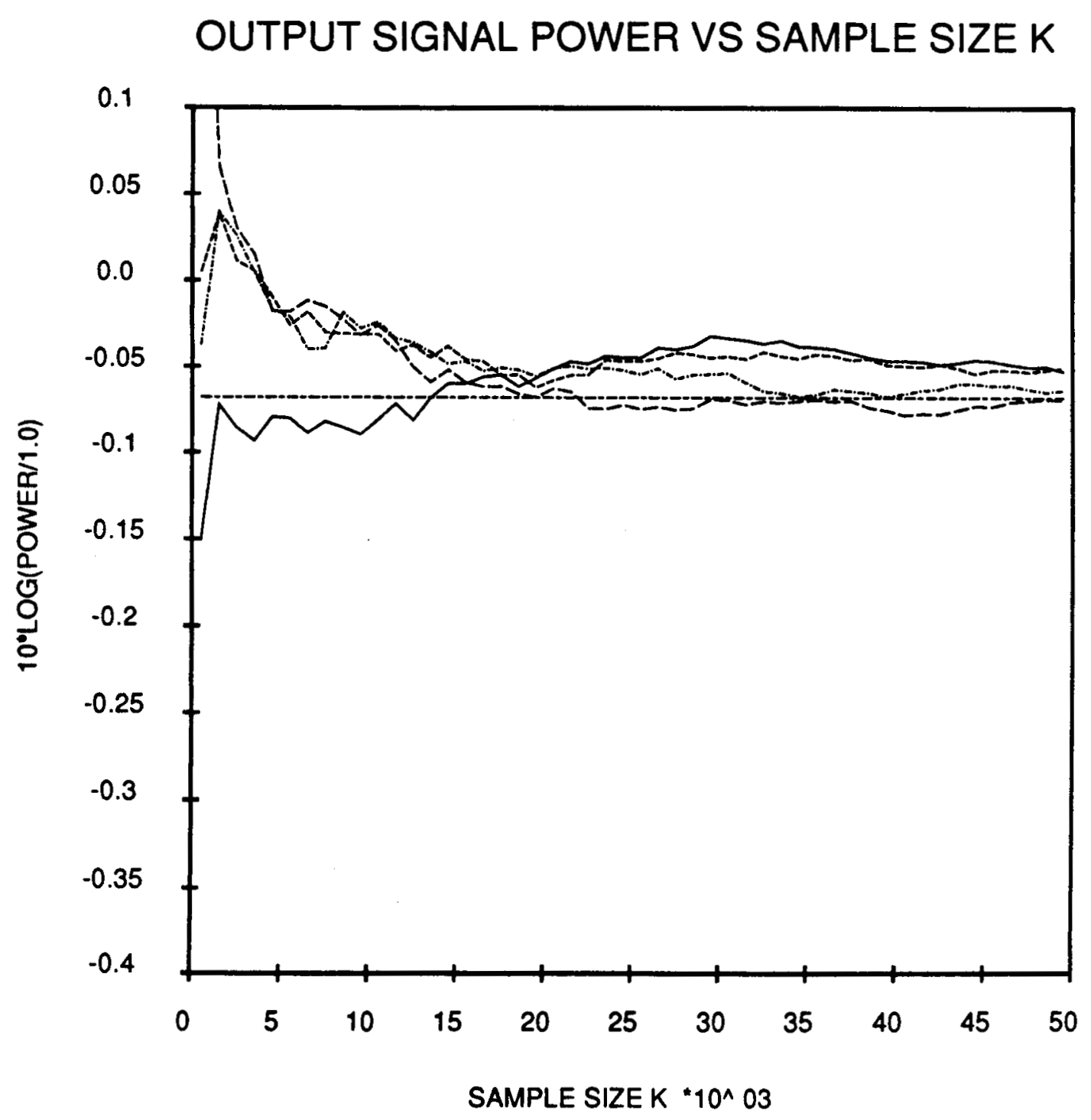

Figure 3.30: Plot of output desired signal power versus number of snapshots $K$ for $F=0.8$ and $\hat{\Phi}_{K}=\Phi+$ DNCT. Shows 4 simulations and the true covariance curve. 


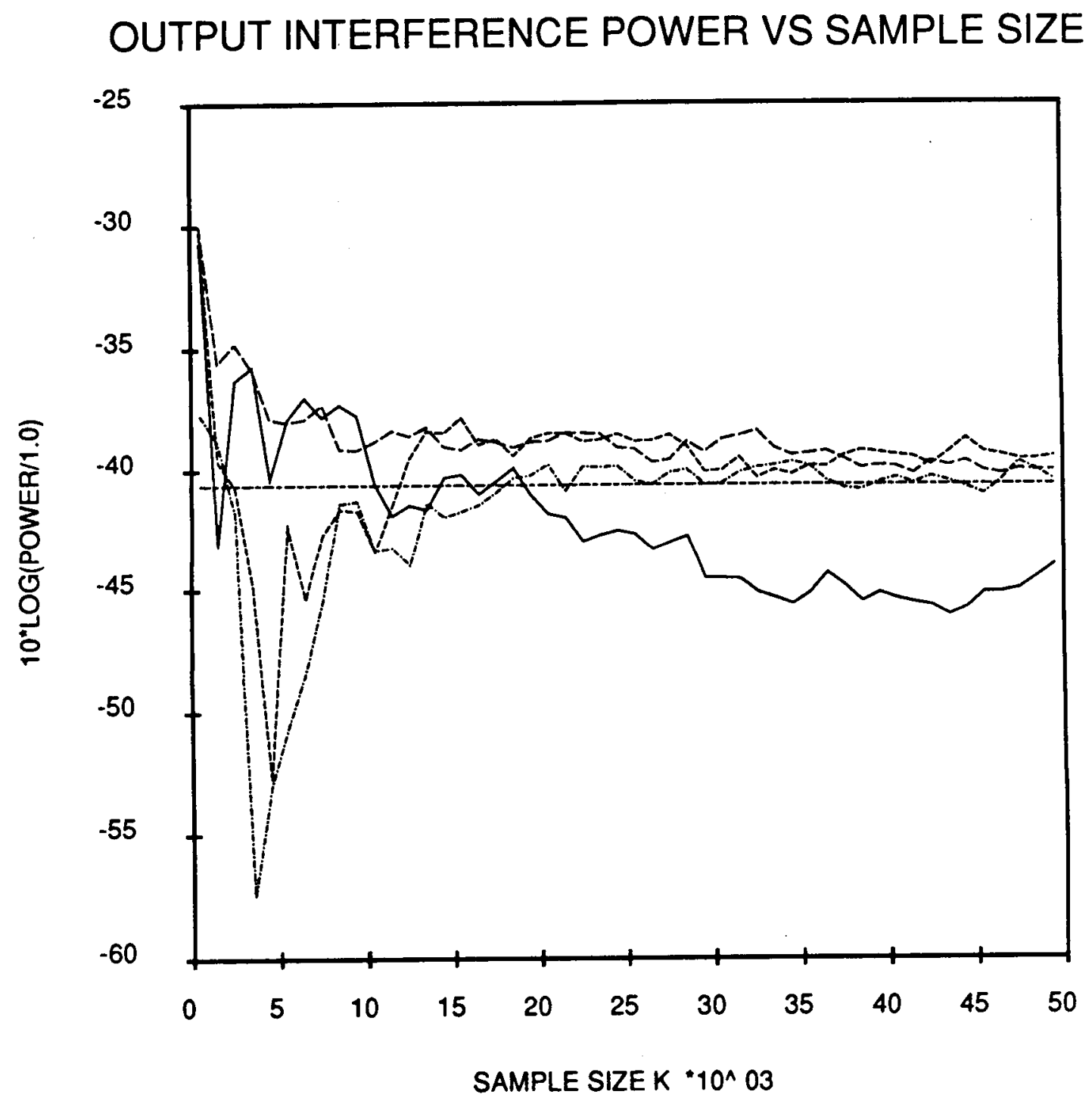

Figure 3.31: Plot of output interference power versus number of snapshots $K$ for $F=0.8$ and $\hat{\Phi}_{K}=\Phi+$ DNCT. Shows 4 simulations and the true covariance curve. 


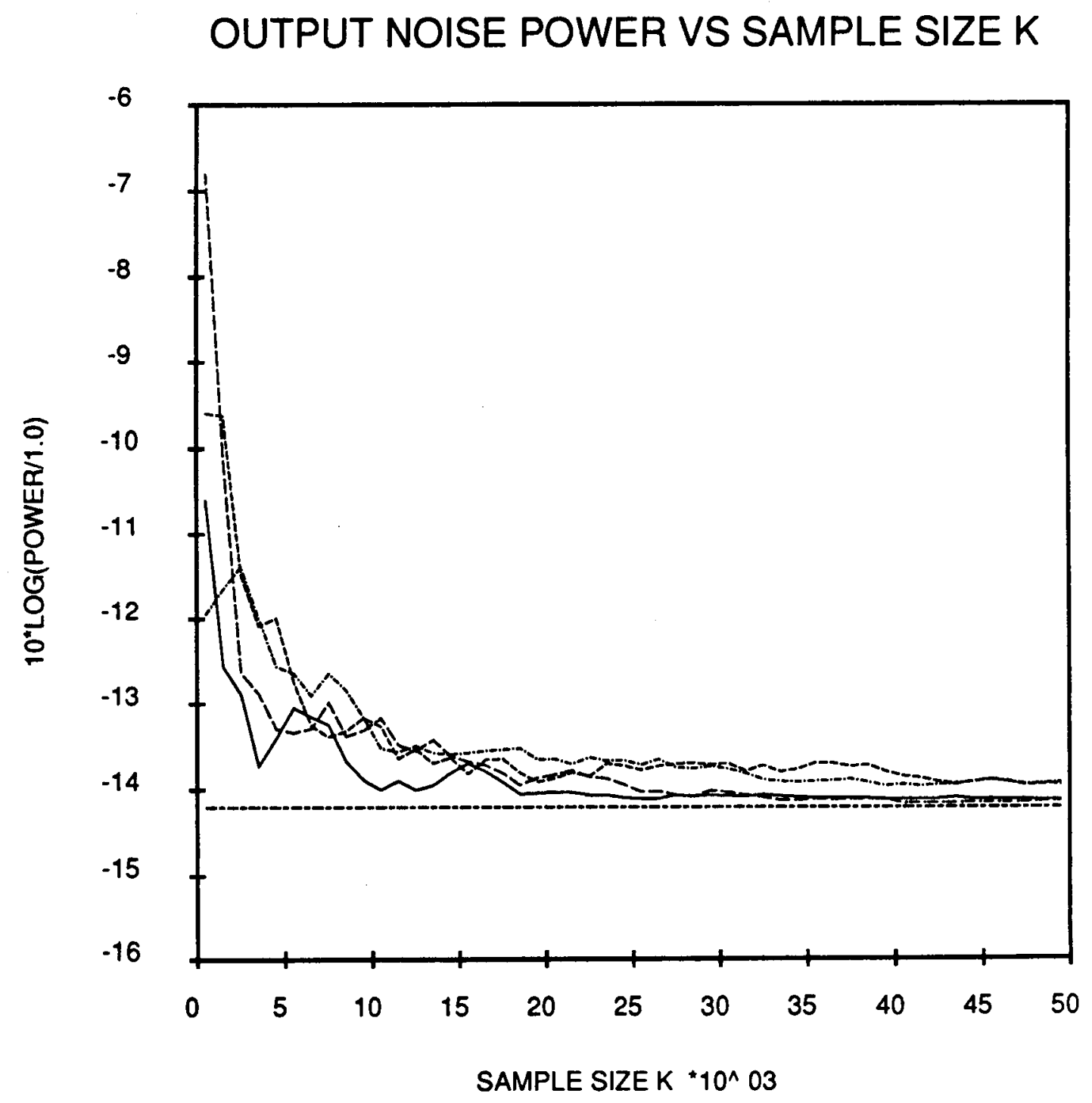

Figure 3.32: Plot of output noise power versus number of snapshots $K$ for $F=0.8$ and $\hat{\Phi}_{K}=\Phi+$ DNCT. Shows 4 simulations and the true covariance curve. 
This observation in turn implies that the most harmful crossterms in $\hat{\Phi}_{K}$ should occur in its first row and first column. To test this, a simulation was run in which the covariance matrix was formed in the normal manner (i.e. with all of its crossterms) and then modified by replacing its first row and column with those of the true covariance $\Phi$. The results of these simulations shown in Figure 3.34 represent near-ideal performance (only small interference power fluctuation) and thus serve to illustrate how a high-gain element is harmful.

\subsection{Summary}

The purpose of this chapter has been to study the performance of the modified SMI algorithm when the true covariance matrix is replaced by an estimate, as is done in practice. First, the sample covariance matrix was defined. Then, statistical theory based on the sample covariance matrix was developed in order to characterize the weight and output power performance of the modified SMI array with fraction $F$ and $K$ available snapshots. It was noted that much of this theory is applicable to any signal scenario including wideband signals. The simulations were introduced by describing the form of a signal snapshot. Next, the minimum eigenvalue of the sample covariance matrix was shown through simulation to be a good estimate of the noise power and hence can be used in practice to implement the diagonal subtraction required in the modified SMI algorithm. The statistical theory was tested by overlaying Monte Carlo simulations with expected value curves and confidence intervals for a particular weak interference scenario 
and various choices of fraction $\mathrm{F}$; good agreement was found. The observation made in the previous chapter, that the true modified SMI weights are not dependent on the noise eigenvectors of the covariance matrix, was followed up in this chapter. Simulations showed that omitting the noise eigenvectors leads to greatly improved output noise power performance but the more crucial interference and desired signal powers are left unimproved. The chapter concluded by studying the severity of different cross-terms that comprise the error in the covariance matrix estimate. This study lent insight into the nature of the weight estimation problem and generated several handy rules-of-thumb. 
OUTPUT INR \& SINR VS SAMPLE SIZE K

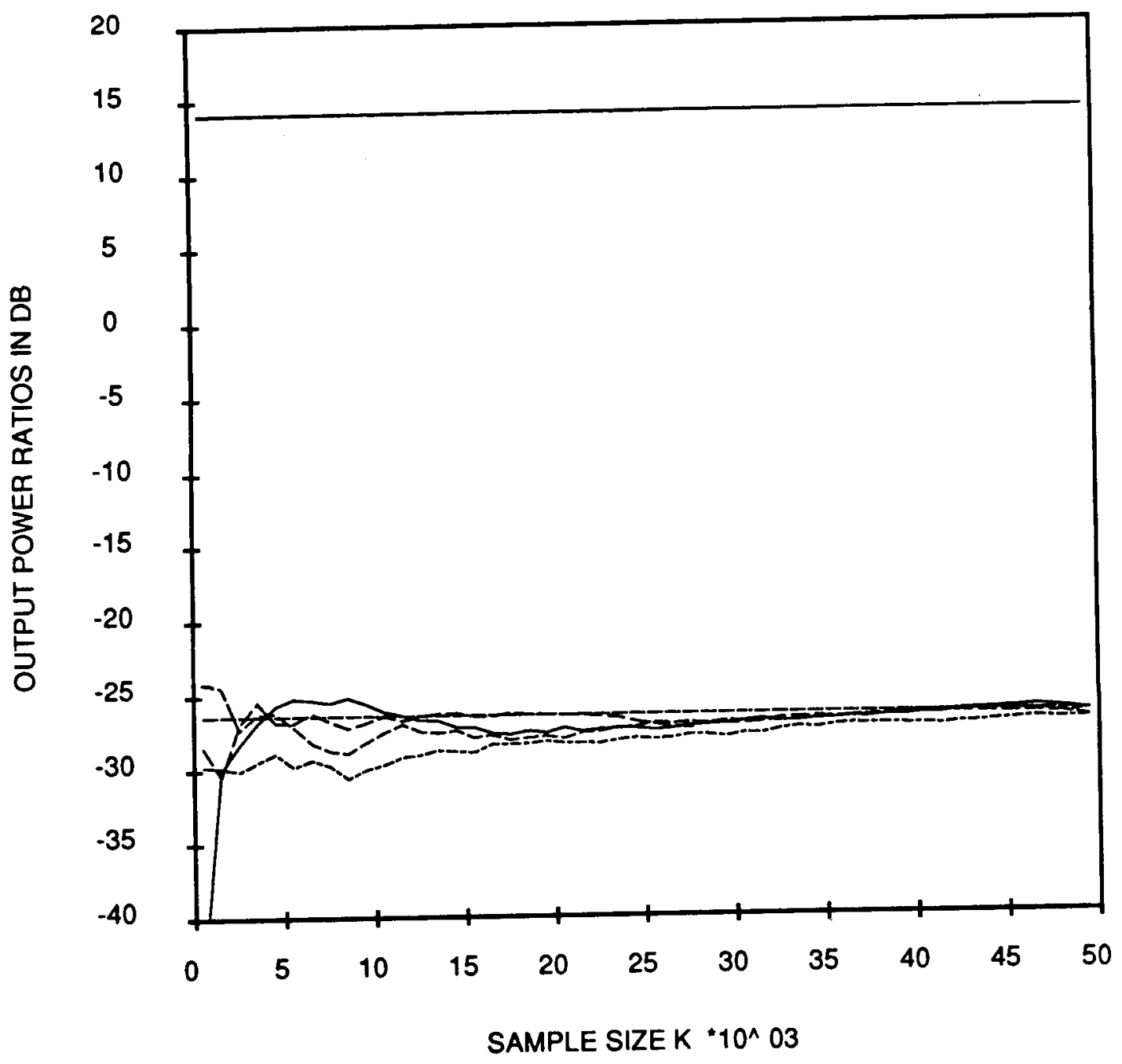

Figure 3.33: Plot of output INR and SINR versus number of snapshots $K$ for $F=0.8$ and $\hat{\Phi}_{K}=\Phi+$ INCT. Shows 4 simulations and the true covariance curve. 


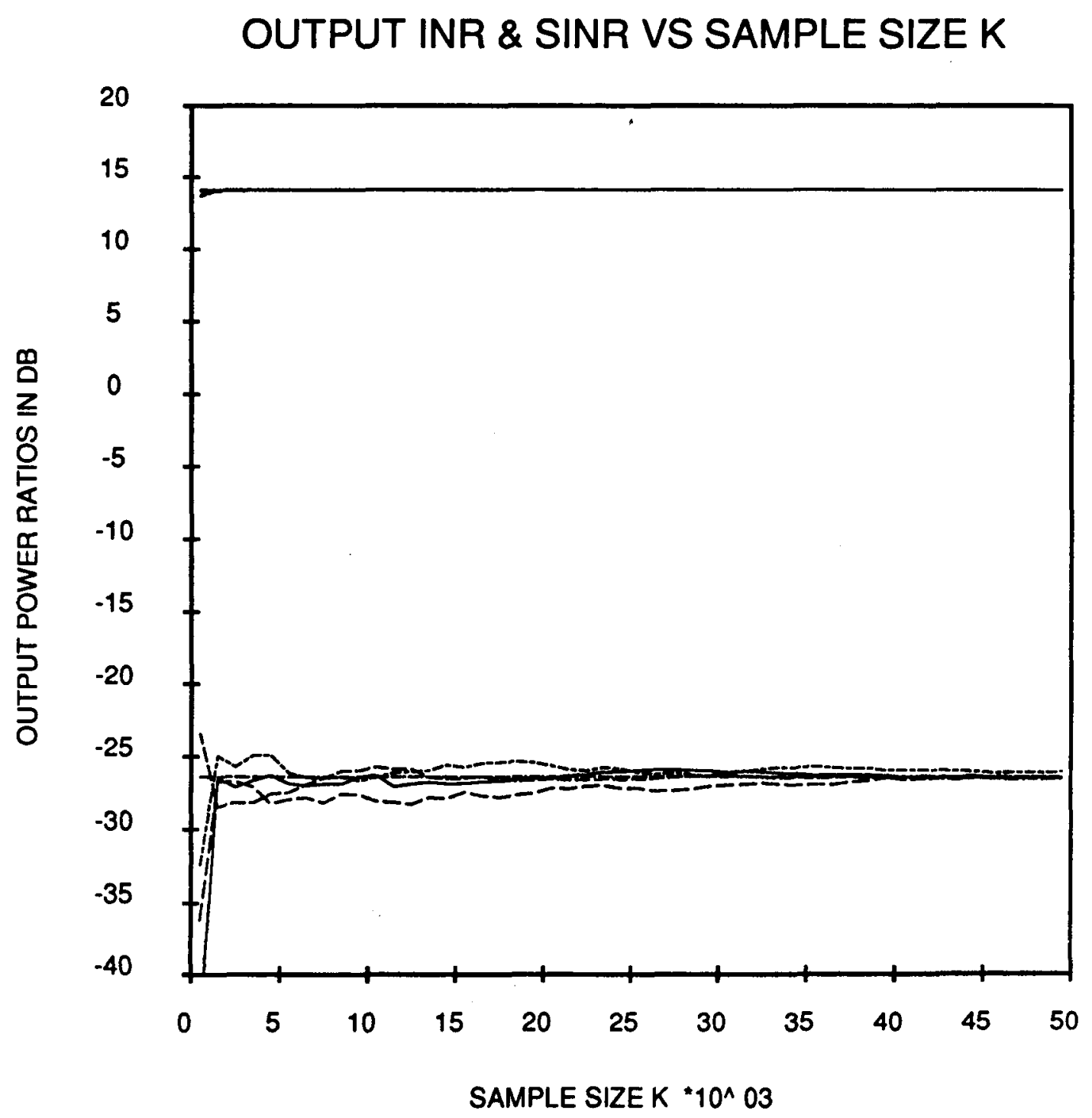

Figure 3.34: Plot of output INR and SINR versus number of snapshots $K$ for $F=0.8$ and $\hat{\Phi}_{K}$ simulated normally (all crossterms) but with its first column and row replaced with those of the true covariance $\Phi$. Shows 4 simulations and the true covariance curve. 


\section{Chapter 4}

\section{Conclusions}

This report has addressed the problem of suppressing weak interference signals while maintaining a strong desired signal by using an adaptive antenna array and a modified version of the SMI weight assignment algorithm. First, the array weights which maximize a modified SINR were derived. The modified SINR is parameterized by a fraction F. As $\mathrm{F}$ ranges from zero to one, the array weights range from the standard SMI weights which maximize the standard SINR to weights which maximize SIR. By choosing F between zero and one, the suppression of weak interference can be significantly enhanced at the modest expense of a slight decrease in SINR. This behavior was analyzed in detail; in particular, the eigen-decomposition of the covariance matrix was used to better understand the properties of the modified SMI algorithm.

In practical applications, the true covariance matrix must be replaced by an estimate formed from a number $\mathrm{K}$ of sample snapshot vectors. For a finite number of snapshots, the array weights are noisy and the performance of the modified SMI method will show a statistical fluctuation from its 
nominal level. The bias and variance of the array weights and output powers as functions of the fraction $\mathrm{F}$ and the number of snapshots $\mathrm{K}$ were derived. These statistical measures allow a designer to determine what, for example, the expected output interference power of the system is as a function of the number of snapshots. It was found that, especially for fractional values $F$ near one, it may take as many as 50,000 snapshots before the expected INR is close to the ideal (true covariance) INR with reasonable statistical confidence. The bias and variance estimates were compared with Monte Carlo simulations and good agreement was observed.

In order to further understand the limiting causes of the degraded performance due to covariance matrix estimation, we studied the effects of each crossterm in the estimate. These crossterms are present whenever a finite number of snapshots are used in the estimate and they approach zero as the number of snapshots increases. It was found that crossterms involving a strong signal lead to a large degradation in the array performance. Crossterms involving noise tend to degrade desired, interference, and noise power performance. Crossterms involving only signals (not noise) tend to substantially degrade interference power performance but not noise power performance. Finally, it was observed that elements in the covariance matrix which involve a high-gain array element (strong signal) tend to have the most harmful error.

The results of this research provide the array designer with theoretical tools to characterize the finite-snapshot performance of the modified SMI system. These theoretical measures were shown to have good agreement with the results obtained by Monte Carlo simulation. Since Monte Carlo 
simulation is computationally expensive, significant savings in computer time can be gained by taking advantage of the theoretical tools provided here. In addition, the theoretical formulas provide a means for analytical manipulations and can be used to investigate array performance as a function of one or more system parameters; this is not possible with Monte Carlo simulation.

The work described in this report suggests two future avenues for research. The first is experimental verification of the modified SMI algorithm on an actual antenna array system to confirm the practical usefulness of the theoretical performance measures. The second avenue of continued research involves finding ways of decreasing the number of snapshots required to achieve a given level of performance. The current modified SMI method was shown to provide acceptable performance for the satellite communication problem using 50,000 snapshots. However, if further interference suppression is needed or if the signal environment is more rapidly changing, then alternate methods for weight estimation are needed. One promising method would directly estimate the parameters (amplitudes and phases) of the true covariance matrix given $\mathrm{K}$ received signal snapshots. This method would make use of additional a priori information concerning the signal scenario left unexploited by the modified SMI algorithm. 


\section{Bibliography}

[1] I. J. Gupta, "SMI Adaptive Antenna Arrays for Weak Interfering Signals," IEEE Transactions on Antennas and Propagation, vol. AP-34, no. 10, pp. 1237-1242, October 1986.

[2] Reed, Mallett, and Brennan, "Rapid Convergence Rate in Adaptive Arrays," IEEE Transactions on Aerospace and Electronic Systems, vol. AES-10, no. 6, pp. 853-863, November 1971.

[3] S. Lawrence Marple, Jr., Digital Spectral Analysis with Applications, Prentice-Hall, Inc., Englewood Cliffs New Jersey, Ch. 13, 1987.

[4] J. Ward, "Adaptive Arrays for Weak Interfering Signals- An Experimental System", M. Sc. Thesis, The Ohio State University, Department of Electrical Engineering, Columbus, OH, 1987.

[5] R. A. Horn and C. A. Johnson, Matrix Analysis, Cambridge University Press, Cambridge, England, p. 301, 1985.

[6] Peter H. M. Janssen and Petre Stoica, "On the Expectation of the Product of Four Matrix-Valued Gaussian Random Variables," IEEE Transactions on Automatic Control, vol. AC-33, no. 9, pp. 867-870, September 1988.

[7] M. Ganz and R. L. Moses and S. Wilson, "Convergence of the SMI and the Diagonally Loaded SMI Algorithms with Weak Interference," IEEE Transactions on Antennas and Propagation, 1988 (submitted). 
[8] R. T. Compton, Jr., "On eigenvalues, SINR, and element patterns in adaptive arrays," IEEE Transactions on Antennas and Propagation, vol. AP-32, no. 6, pp.643-647, June 1984.

[9] N. R. Goodman, "Statistical analysis based on a certain multivariate complex Gaussian distribution," Ann. Math. Stat., vol. 34, pp. 152177, March 1963. 